\title{
Estimation of Alkali Metal Mole Percent and Weight of Calcined Solids for ICPP Calcine
}

B. H. O'Brien

Published March 1995

Idaho National Engineering Laboratory

High-Level Waste Engineering and Project Management Department Lockheed Idaho Technologies Company Idaho Falls, Idaho 83415

Prepared for the

U.S. Department of Energy

Assistant Secretary for Environmental Management

Under DOE Idaho Operations Office

Contract DE-AC07-94ID13223 


\section{DISCLAIMER}

Portions of this document may be illegible electronic image products. Images are produced from the best available original document. 
An updated method is given for estimation of the weight of calcined solids and volume reduction factor for calcine, and mole percent sodium plus potassium in calcine produced from radioactive waste in a fluidized-bed calciner at the Idaho Chemical Processing Plant (ICPP). It incorporates new information on a calcine chemistry from a study by $\mathrm{K}$. N. Brewer and G. F. Kessinger in which they determined the compounds formed during calcination by both high temperature thermodynamic equilibrium calculations and by analyses of pilot-plant calcines. An explanation of the assumptions made in the calculations, along with several example calculations and comparisons with the previous calculation methods are included. This method allows calculation of the heat generation rate and sodium content of the calcine, which are used to determine the suitability of the calcine for storage in the ICPP bin sets. Although this method accurately predicts the weight of calcine and mole percent $\mathrm{Na}+\mathrm{K}$ for its intended purpose, the compounds predicted should only be used as a first approximation for other purposes since the calculation does not incorporate all of the compounds, such as mixed-metal oxides, which may form during calcination.

\section{DISCLAIMER}

This report was prepared as an account of work sponsored by an agency of the United States Government. Neither the United States Government nor any agency thereof, nor any of their employees, makes any warranty, express or implied, or assumes any legal liability or responsibility for the accuracy, completeness, or usefulness of any information, apparatus, product, or process disclosed, or represents that its use would not infringe privately owned rights. Reference herein to any specific commercial product, process, or service by trade name, trademark, manufacturer, or otherwise does not necessarily constitute or imply its endorsement, recommendation, or favoring by the United States Government or any agency thereof. The views and opinions of authors expressed herein do not necessarily state or reflect those of the United States Government or any agency thereof. 


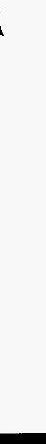




\section{SUMMARY}

An updated method is given for estimation of the weight of calcined solids and volume reduction factor for calcine, and mole percent sodium plus potassium in calcine produced from radioactive waste in a fluidized bed calciner at the Idaho Chemical Processing Plant. This method is a simplification of the complex chemical reactions which occur during calcination and allows calculation of the heat generation rate and sodium content of the calcine, which are used to determine the suitability of the calcine for storage in the ICPP Calcined Solids Storage Facilities (CSSFs). Although this method accurately predicts the weight of calcine, weight percent of elements in the calcine, and mole percent of $\mathrm{Na}+\mathrm{K}$ in calcine for its intended purpose, the compounds predicted should only be used as a first approximation for other purposes since the calculation does not incorporate all of the compounds, such as bimetallic oxides, which may form during calcination.

The previous method for calculating the weight of calcined solids from the feed composition was primarily designed for use with raffinates from fuel dissolution and was inadequate for blends where there is insufficient fluoride to form $\mathrm{Na}_{3} \mathrm{AlF}_{6}$, such as in cold aluminum nitrate/sodium waste blends. In addition, the previous method for calculating the sodium plus potassium mole percent in calcine did not use all of the feed components used in the solids calculation, and also did not account for the nitrate present in the calcine. These previous methods had been used to verify suitability of calcine for storage in Calcined Solids Storage Facilities 5 and 6 while the updated method will be used for calcine stored in CSSF 6 and future Calcined Solids Storage Facilities (CSSFs) after NWCF campaign H-3.

The updated method assumes the same compounds are formed for both calculation of weight of calcined solids and sodium plus potassium mole percent of calcine. It incorporates new information on calcine chemistry from a study by K. N. Brewer and G. F. Kessinger in which they determined the compounds formed during calcination by both high temperature thermodynamic equilibrium calculations and by analyses of pilot plant calcines. An explanation of the assumptions made in the calculations, along with several example calculations and comparisons of calculated values with values using the previous calculation methods are attached. 


\section{ACKNOWLEDGEMENTS}

This work combines new information from a study by K. N. Brewer and G. F. Kessinger with earlier work by a number of people, principle among them being B. J. Newby, W. B. Palmer, and J. R. Berreth. The author would especially like to thank $\mathrm{K}$. N. Brewer for his assistance and helpful comments. 



\section{CONTENTS}

ABSTRACT $\ldots \ldots \ldots \ldots \ldots \ldots \ldots \ldots \ldots \ldots \ldots \ldots \ldots \ldots \ldots \ldots \ldots \ldots \ldots$

$\operatorname{SUMMARY} \ldots \ldots \ldots \ldots \ldots \ldots \ldots \ldots \ldots \ldots \ldots \ldots \ldots \ldots \ldots \ldots \ldots$

ACKNOWLEDGEMENTS $\ldots \ldots \ldots \ldots \ldots \ldots \ldots \ldots \ldots \ldots \ldots \ldots \ldots \ldots$ vii

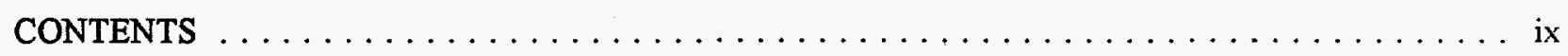

1. INTRODUCTION $\ldots \ldots \ldots \ldots \ldots \ldots \ldots \ldots \ldots \ldots \ldots \ldots \ldots \ldots \ldots \ldots$

2. COMPOUNDS FORMED DURING CALCINATION $\ldots \ldots \ldots \ldots \ldots \ldots \ldots \ldots \ldots$

2.1 Nitrates . . . . . . . . . . . . . . . . . . 3

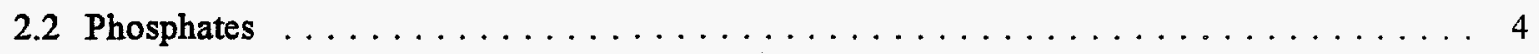

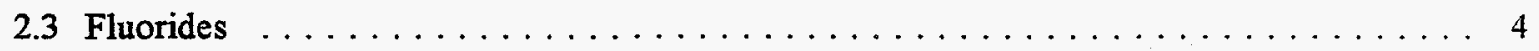

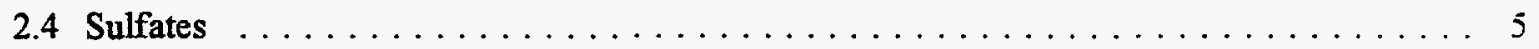

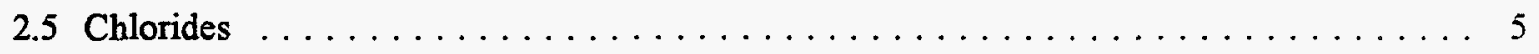

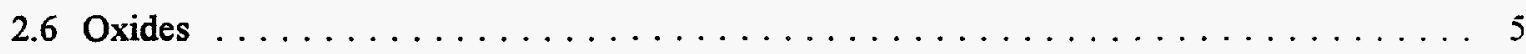

2.7 Undissolved Solids $\ldots \ldots \ldots \ldots \ldots \ldots \ldots \ldots \ldots \ldots \ldots \ldots \ldots \ldots \ldots \ldots \ldots \ldots$

2.8 Minor Feed Components $\ldots \ldots \ldots \ldots \ldots \ldots \ldots \ldots \ldots \ldots \ldots \ldots \ldots \ldots \ldots \ldots \ldots$

3. CALCULATION METHODS $\ldots \ldots \ldots \ldots \ldots \ldots \ldots \ldots \ldots \ldots \ldots \ldots \ldots \ldots \ldots$

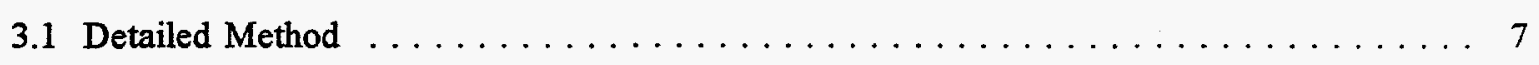

3.2 Simplified Method $\ldots \ldots \ldots \ldots \ldots \ldots \ldots \ldots \ldots \ldots \ldots \ldots \ldots \ldots$

3.3 Summary of Calculation Method $\ldots \ldots \ldots \ldots \ldots \ldots \ldots \ldots$

4. COMPARISON OF CALCULATED VALUES WITH PREVIOUS METHODS $\ldots \ldots \ldots \ldots$

5. EFFECTS OF NEGLECTING TRACE OR UNKNOWN CONCENTRATIONS OF FEED

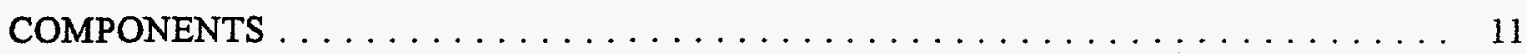


6. CALCULATIONAL ERROR

7. REFERENCES

Appendix A

Sample Detailed Calculation of Sodium Plus Potassium Content

and Weight of Calcined Solids

A-1

Appendix B

Verification of Simplified Equation for Moles of Oxygen

B-1

Appendix $\mathrm{C}$

Example Calculations .

C-1

Appendix D

Comparision of Updated Calculation Method

with Previous Methods

D-1

Appendix E

Calculation of Liquid Waste Decay Heat

E-1 


\section{INTRODUCTION}

The weight of solids produced during calcination is needed to predict the volume of calcine which will be produced for calcine storage. The calcined solids weight, along with its specific volume, are also needed to predict the heat generation rate of the calcine to ensure that temperatures in the Calcined Solids Storage Facilities (CSSFs) do not exceed the temperature at which the calcine will cake. In addition; the caking tendency of calcine is dependent on the alkali metal content of the calcine. A method for estimating the compounds formed during calcination is necessary to determine the weight of solids produced during calcination and the alkali metal content of the calcine.

The previous method for calculating the weight of solids produced during calcination (Reference 1) assumed that the sodium and potassium in the calcine formed fluoaluminates $\left(\mathrm{Na}_{3} \mathrm{AlF}_{6}\right.$ and $\left.\mathrm{K}_{3} \mathrm{AlF}_{6}\right)$; however, this assumption is not applicable in cases where insufficient fluoride is available, such as in aluminum nitrate/sodium waste blends. In addition, the method for calculating alkali metal (sodium plus potassium) mole percent in calcine (Reference 2) assumed slightly different compounds were formed than Reference 1, did not use all the feed components used in the calcined solids calculation method of Reference 1, and also did not account for nitrate formation in the calcine. An updated calculation method which uses the same chemistry and compounds formed for calculating both the calcined solids produced and mole percent alkali metals in the calcine, and which incorporates information from a new study of calcination chemistry, has been developed.

The updated method incorporates information from a comprehensive study of calcination chemistry by K. N. Brewer and G. F. Kessinger (References 3 and 4) in which calcination chemistry was determined by both thermodynamic equilibrium calculations and analysis of pilot-plant calcine using SEM, $x$-ray diffraction, and elemental analysis.

The methods in References 1 and 2 have been used to verify compliance of New Waste Calcining Facility (NWCF) feed blends with the Technical Specifications for calcine storage in CSSF 5 and 6. The updated method will be used to verify compliance of future NWCF waste blends, particularly high sodium content blends of aluminum nitrate and sodium waste, with the operating limits in the new revisions of the technical standards for calcine storage in CSSFs 6 and 7. In addition, the values for weight of calcined solids and associated volume reduction factors are used by operations 
personnel to estimate the volume of calcine transferred to the CSSFs from the amount of feed processed at the NWCF. Although this method accurately predicts the weight of calcine and mole percent $\mathrm{Na}+\mathrm{K}$ for its intended purpose, the compounds predicted should only be used as a first approximation for other purposes such as prediction of ceramic forms in the calcine since the calculation does not incorporate all of the compounds, such as bimetallic oxides, which may form during calcination. For example, sodium oxide $\left(\mathrm{Na}_{2} \mathrm{O}\right)$ is very reactive and would be expected to exist in the calcine as sodium aluminate $\left(\mathrm{Na}_{2} \mathrm{O} \cdot \mathrm{xAl}_{2} \mathrm{O}_{3}\right)$ or another more thermodynamically favorable bi- or tri-metallic oxide (Reference 5). In addition, the compounds formed are to some extent dependent on the feed composition.

The method can be used to predict weight percents of individual elements in the calcine such as calcium and nitrate.

\section{COMPOUNDS FORMED DURING CALCINATION}

Calcination is a very complex chemical process due to the large number of chemical species and wide variation in chemical concentrations in the calciner feed. Crystalline compounds in calcine can be determined by $x$-ray diffraction methods; however, the majority of compounds in calcine are amorphous (non-crystalline) and usually the concentrations of only a few crystalline compounds are above the detection limits. A program which calculates the chemical equilibrium, such as the $F^{*} A^{*} C^{*} T$ EQUILIB program (Reference 6) used by K. N. Brewer and G. F. Kessinger, can be used to estimate the distribution of compounds formed at equilibrium. Unfortunately, the fluidized bed calciner is not an equilibrium process due to the finite residence time in the calciner, local temperature variations in the bed, and continuously changing operating conditions such as feed rate and fuel rate. For example, the thermodynamic calculations predicted that no nitrates would remain at equilibrium while analyses of calcine have shown that nitrates are present.

A brief description of the assumptions and simplifications used in the calculation method are listed below. These assumptions are based primarily on the work of $K . N$. Brewer and $G$. F. Kessinger (References 3 and 4), and on an examination of the free energies and heats of formation for potential compounds. K. N. Brewer and G. F Kessinger determined calcine composition by thermodynamic 
equilibrium calculations using the $F^{*} A^{*} C * T$ EQUILIB program, and compared the results with analyses of pilot plant calcines.

For the purposes of the calculation, nitrates are assumed to form first, followed by phosphates, fluorides, sulfates, chlorides, and then oxides. This assumption is a simplification that allows the calculation to be made in a non-iterative fashion.

\subsection{Nitrates}

A fixed mole fraction of the sodium and potassium is assumed to form nitrates in the calcine. For pilot plant calcine, this value was calculated to be an average of $0.66 \pm 0.05$ moles of nitrate per mole of sodium plus potassium (Reference 7), and this mole ratio is used to calculated nitrate in the calcine. The remaining nitrate present in the feed is conservatively assumed to volatilize. The nitrate content of calcine varies inversely with calcination temperature for the same feed composition (Reference 8); however, the calciner pilot plant and NWCF operating temperatures do not vary significantly from $500^{\circ} \mathrm{C}$. Reducing agents have been successfully tested in pilot plant calciners to reduce the amount of nitrates in the calcine (Reference 9). The average value of 0.66 moles nitrate per mole of sodium is not applicable for instances where reducing agents such as sugar have been used to destroy the nitrates in the calciner feed; however, these types of feed additives are not currently used at the NWCF. If reducing agents or other chemicals expected to affect the nitrate content of the calcine are to be used, then pilot plant or plant data for that feed composition and additives should be used to estimate the nitrate content of the calcine for the calculation.

The previous method for calculation of sodium plus potassium content (Reference 2) neglected nitrate formation, while the method for calculation of calcined solids (Reference 1) assumed that $10 \%$ of the sodium and potassium and $50 \%$ of the excess calcium existed as nitrates. The new value of $66 \%$ of the sodium plus potassium existing as nitrates is based on pilot plant data and should be more accurate than the old value of $10 \%$. For calcium nitrate, the thermodynamic equilibrium calculations performed by Brewer and Kessinger (References 3 and 4) did not predict that calcium nitrate would form, and in addition, the nitrate content of pilot plant calcines does not show a strong correlation with the excess calcium (Reference 7). Therefore, all the excess calcium (after formation of phosphates, fluorides, sulfates, and chlorides) was assumed to form oxides in the updated calculation method. 


\subsection{Phosphates}

Calcium phosphate is assumed to form first, followed by sodium and potassium phosphate, based on the equilibrium calculations. If there is insufficient calcium, sodium and potassium, the remaining phosphate is conservatively assumed to volatilize.

The previous calculation of sodium plus potassium content (Reference 2) neglected phosphate since the measured phosphate in tank farm wastes is small. Historically, the phosphate content in tank farm wastes has never exceeded $0.028 \underline{\mathrm{M}}$ (Reference 10). The previous calculation method for calcined solids (Reference 1) assumed that $50 \%$ of the phosphate volatilized; however, in pilot plant tests which burned tri-butyl-phosphate-containing process solvent as fuel the majority of the phosphate stayed in the calcine solids and less than $1 \%$ volatilized as determined by analyses of the acid scrub and off-gas (Reference 11). Brewer's equilibrium calculations (Reference 4) indicated that sodium and potassium phosphate could form, but only if there was insufficient calcium.

\subsection{Fluorides}

Calcium fluoride $\left(\mathrm{CaF}_{2}\right)$ is assumed to preferentially form, followed by $\mathrm{NaF}$ and $\mathrm{KF}$, based on equilibrium calculations (Reference 3 ). If insufficient calcium, sodium and fluoride are available for these compounds, the excess fluoride is conservatively assumed to volatilize.

In both of the previous calculations (References 1 and 2), $\mathrm{Na}_{3} \mathrm{AlF}_{6}$ and $\mathrm{K}_{3} \mathrm{AlF}_{6}$ were assumed to form preferentially to $\mathrm{CaF}_{2}$; however, $\mathrm{K}$. N. Brewer's equilibrium calculations and analyses indicated that most of the fluoride forms $\mathrm{CaF}_{2}$, with only small amounts of $\mathrm{NaF}$ and $\mathrm{KF}$, and no $\mathrm{Na}_{3} \mathrm{AlF}_{6}$ or $\mathrm{K}_{3} \mathrm{AlF}_{6}$.

Fluoride volatility is routinely measured during pilot plant tests and only flowsheets which have fluoride volatility of less than $1 \%$ are recommended for operation at NWCF. For Fluorinel/Sodium waste blends and Aluminum/Fluorinel/Sodium blends in the Enclosed 30-cm Calciner Pilot Plant, the fluoride volatility ranged from 0.1 to $0.5 \%$ (Reference 12). Therefore, the assumption that all fluoride is tied up by $\mathrm{Ca}, \mathrm{Na}$, and $\mathrm{K}$ is reasonable, especially since a calcium to fluoride ratio of 0.55 or greater is required for NWCF operation with fluoride blends (Reference 13) and because the sodium concentration in current sodium wastes is much greater than the fluoride concentrations in those wastes. 


\subsection{Sulfates}

Potassium sulfate is assumed to form, followed by sodium sulfate, calcium sulfate, and then cadmium sulfate. If insufficient $\mathrm{K}, \mathrm{Na}, \mathrm{Ca}$ and $\mathrm{Cd}$ is available for sulfate formation, then any excess sulfate is conservatively assumed to volatilize.

The previous methods assumed that cadmium sulfate would form first, but the free energy for cadmium sulfate is less favorable than that for potassium, sodium, or calcium sulfate. The thermodynamic equilibrium calculations also favored potassium, sodium and calcium over cadmium sulfate, but the lab analyses of pilot plant calcine by $x$-ray diffraction in the $\mathrm{K}$. N. Brewer's study indicated the presence of $\mathrm{CdS}$. It is unlikely that $\mathrm{SO}_{4}$ in the feed would dissociate. It is possible the sulfur could be from the small amount of sulfur (< $0.04 \mathrm{wt} \%)$ present in the kerosene used as fuel in the calciners. If $\mathrm{CdS}$ does form from the sulfur present in the kerosene instead of $\mathrm{CdO}$, the total number of elemental moles in the calcine and hence the mole \% sodium plus potassium would be unchanged. Since the molecular weight of $\mathrm{CdO}$ is less than $\mathrm{CdS}$, the assumption that $\mathrm{CdO}$ is formed will give a lower calculated weight of calcined solids and will provide a conservatively high calculated volume reduction factor in corresponding calcine heat generation rate.

\subsection{Chlorides}

Potassium chloride and sodium chloride are assumed to form before calcium chloride based on the thermodynamic equilibrium calculations. If there is not enough potassium, sodium, and calcium, the excess chloride is conservatively assumed to volatilize. The previous methods assumed that only $\mathrm{CaCl}_{2}$ was formed.

\subsection{Oxides}

Sodium and potassium are assumed to form $\mathrm{NaAlO}_{2}$ if there is enough aluminum. Any remaining sodium, potassium, and other metals are treated as their respective metal oxides. Many of the metals, including sodium and especially potassium, probably exist in calcine as mixed metal oxides such as $\mathrm{KBO}_{2}, \mathrm{NaBO}_{2}, \mathrm{NaCrO}, 3 \mathrm{CaO} \cdot \mathrm{B}_{2} \mathrm{O}_{3}$ and $\mathrm{CdO} \cdot \mathrm{Al}_{2} \mathrm{O}_{3}$; however, the oxides are treated as separate 
compounds in the calculations since: 1) this does not affect the total moles of metals and oxygen present, and 2) the exact oxide compounds which form can change based on the relative mole ratios in the feed. While the use of individual oxides is adequate for the above mentioned purposes, it does not provide the exact compounds formed during calcination in all cases, and as such should only be used as a first approximation if such information is desired. For a more detailed discussion of mixed metal oxides which may form, see References 3 and 5 .

\subsection{Undissolved Solids}

The undissolved solids (UDS) in the feed are assumed to remain solid in the calcine and are added to the weight of calcine produced, but are not used for calculation of the sodium plus potassium content. This assumption is the same as that used in the previous calculation methods.

\subsection{Minor Feed Components}

Over $99 \%$ of mercury in the feed volatilizes during calcination at $500^{\circ} \mathrm{C}$ (Reference 15 ), so mercury is not included in the calculations. As a cautionary note, a significant portion of mercury did remain in the calcine during early calcination at ICPP when indirect heating and calcination temperatures of $400^{\circ} \mathrm{C}$ were used.

Minor feed components with concentrations less than $0.5 \mathrm{~g} / \mathrm{L}$ are neglected. This is conservative since neglecting feed components (other than sodium and potassium) reduces the total weight and total moles for calcine produced. This results in a conservatively low weight of calcined solids with a corresponding conservatively high calcine heat generation rate, and a conservatively high sodium plus potassium mole percent. If the concentration of a feed component is known to exceed $0.5 \mathrm{~g} / \mathrm{L}$ in the feed blend, then it can be included by assuming that its oxidation state in the calcine is the same as that in the feed and that it forms an oxide. Although they are usually less than $0.5 \mathrm{~g} / \mathrm{L}$ in calciner feed, the toxic metals cadmium, chromium, and lead, and also uranium are included in the calculation because their content in calcine is usually desired for waste management purposes. 


\section{CALCULATION METHODS}

\subsection{Detailed Method}

The detailed method for calculating sodium plus potassium mole percent in calcine, the weight of solids produced per liter of feed, and the volume reduction factor are given in Appendix A, along with a sample calculation. A list of the compounds which are assumed to be formed, and the order of preference for formation is also listed in Appendix A.

In Appendix A, the moles of each individual compound are calculated, and then the oxygen in oxides is summed to determine the total moles of oxygen in the calcined solids. The moles and weights of each element or species in the calcine, including oxides and nitrates, are then summed to determine the total moles in the calcine and the total weight of calcine, respectively. Amounts of elements which were calculated to volatilize are subtracted from the sums.

\subsection{Simplified Method}

An alternate method, which has been used previously (Reference 5), and is simpler than calculating the moles of each individual compound, is to calculate the moles of oxygen in oxides from the sum of the moles of the other elements in the calcine times their oxidation states. The sum of the oxidation states for all the elements in the calcine must sum to zero, otherwise the calcine would be ionically charged. The amounts of each element which remain after formation of nitrates, phosphates, fluorides, sulfates, and chlorides are assumed to form oxides and for the sum of charges to balance, with oxygen having an oxidation state of (-2), then:

$$
(-2) \text { moles }_{\text {axygen }}+\sum_{i=1}^{\text {Components }}\left(\left(\text { Oxidation State }_{i}\right)\left(\text { moles }_{i}\right)\right)=0
$$

Thus, the moles of oxygen in the calcine can be calculated without having to determine the moles of each individual compound formed. Solving for moles of oxygen, equation (1) rearranges to: 


$$
\text { moles }_{\text {axygen }}=\frac{1}{2}\left(\sum_{i=1}^{\text {Components }}\left(\left(\text { Oxidation State }_{i}\right)\left(\text { moles }_{i}\right)\right)\right)
$$

For the elements used in the calculation in Appendix A, equation 2 becomes:

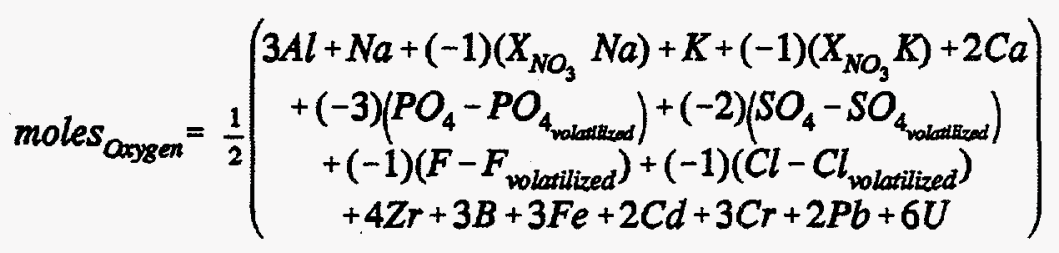

where: $\mathrm{Al}, \mathrm{Na}, \mathrm{K}$, etc. are the moles of each component per liter of feed.

$\mathrm{X}_{\mathrm{NO3}}$ is the mole fraction of sodium and potassium that forms nitrates in the calcine.

To determine the amounts of species which volatilize, the moles of each individual compound must be determined, as in Appendix A. However, if there is sufficient calcium, sodium, and potassium to tie up all the phosphate, fluoride, sulfate, and chloride, then the amounts of these species which volatilize will be zero. For this to be true, then there must be sufficient calcium, sodium, and potassium for all the phosphate, fluoride, sulfate, and chloride:

$$
\left[2 \mathrm{Ca}+\left(1-\mathrm{X}_{\mathrm{NO}_{3}}\right)(\mathrm{Na}+\mathrm{K})\right] \geq\left(3 \mathrm{PO}_{4}+\mathrm{F}+2 \mathrm{SO}_{4}+\mathrm{Cl}\right)
$$

If equation (4) is true, then there will be sufficient $\mathrm{Na}, \mathrm{K}$, and $\mathrm{Ca}$ to form sulfates, so there will be no sulfate available to form cadmium sulfate.

If equations (4) is not true for a given feed composition, then some species will possibly volatilize, in which case the detailed method in Appendix A must be used instead of the simplified method. If equations (4) is true, then there is enough $\mathrm{Na}, \mathrm{K}$, and $\mathrm{Ca}$ to combine with all the $\mathrm{PO}_{4}, \mathrm{~F}$, $\mathrm{SO}_{4}$, and $\mathrm{Cl}$, and none of them will volatilize. Equation (3) then becomes: 


$$
\text { moles }_{\text {Oxygen }}=\frac{1}{2}\left(\begin{array}{c}
3 \mathrm{Al}+\mathrm{Na}+(-1)\left(\mathrm{X}_{\left.\mathrm{NO}_{3} \mathrm{Na}\right)+\mathrm{K}+(-1)\left(\mathrm{X}_{\mathrm{NO}_{3}} \mathrm{~K}\right)+2 \mathrm{Ca}}+(-3) \mathrm{PO}_{4}+(-2) \mathrm{SO}_{4}\right. \\
+(-1) \mathrm{F}+(-1) \mathrm{Cl} \\
+4 \mathrm{Zr}+3 \mathrm{~B}+3 \mathrm{Fe}+2 \mathrm{Cd}+3 \mathrm{Cr}+2 \mathrm{~Pb}+6 \mathrm{U}
\end{array}\right)
$$

It can be shown that for all cases where equation (4) is true that the calculation method in Appendix $A$ and equation (5) result in the same value for moles of oxygen. Appendix $B$ shows the derivation of equation (5) from the calculation method in Appendix A for several cases. If equation (4) is not all true, then it is uncertain whether any $\mathrm{PO}_{4}, \mathrm{~F}, \mathrm{SO}_{4}$, or $\mathrm{Cl}$ will volatilize, and the method in Appendix A must be used to determine the moles of oxygen in the calcine.

Once the total moles of oxygen is calculated using equation (5), then the moles and weights of each element in the calcine can be summed to determine the total moles and the total weight of calcine, respectively.

\subsection{Summary of Calculation Method}

In summary, to determine mole percent sodium plus potassium and weight of calcined solids:

1. Determine if equations (4) is true.

2. If equation (4) is true, then use equation (5) to calculate moles of oxygen, otherwise use the method in Appendix A.

3. Calculate the mole percent sodium plus potassium by dividing the moles of sodium plus potassium by the sum of moles in the calcine, including oxides and nitrates. Moles of nitrate, phosphate, and sulfate are used in the calculation of total moles rather than the moles of individual elements for these species.

4. Calculate the weight of calcined solids either by summing the molecular weight times the moles of individual elements and species, including the weight of oxygen and nitrate, or alternatively by summing the molecular weights times moles for all the compounds formed.

5. Obtain the bulk density of calcine product from either plant or pilot plant data from 
calcination of a similar feed.

6. Estimate the bulk density of the product and fines mixture as a percentage of the product bulk density based on the expected product-to-fines ratio from pilot plant tests.

For most cases, use $95 \%$ of the product bulk density. If the product-to-fines ratio is less than 2, use $90 \%$ of the product bulk density. See figure G-1 in ENICO-1100 (Reference 1).

7. From the bulk density and weight of solids produced, calculate the volume reduction factor as volume of calciner feed to volume of calcine produced.

8. Since cold chemicals such as aluminum nitrate are not normally included in the volume reduction factor, adjust the volume reduction factor to be the ratio of tank farm solution volume to volume of calcine produced.

9. a. Determine the heat generation rate for the liquid feed blend (see Appendix E), then apply the volume reduction factor from step 7 to determine the heat generation rate for the calcined solids.

or b. Determine the heat generation rates for the tank farm liquid (see Appendix E), then apply the volume reduction factor from step 8 to determine the heat generation rate for the calcined solids.

Example calculations of sodium plus potassium content, weight of calcined solids, volume reduction factor, and calcine heat generation for several typical feed compositions are given in Appendix C. Calculation of liquid and calcine heat generation rates is given in Appendix E.

\section{COMPARISON OF CALCULATED VALUES WITH PREVIOUS METHODS}

B. J. Newby used a 5:1 blend of 'typical' Fluorinel and Sodium wastes as the first example calculation of mole percent sodium plus potassium in Reference 2. The value for mole percent sodium plus potassium for this blend the updated method is 4.75 mole \% (see Appendix D) while B. J. Newby's method gives a slightly higher value of 4.84 mole \%. This is a relative difference of $2.0 \%$. The value calculated by the updated method is lower because the total moles is increased by the addition of nitrates and other species such as iron and chloride which B. J. Newby assumed were negligible. 
Since values of mole percent sodium plus potassium calculated using B. J. Newby's method are available for previous blends, it is useful to have a quick way to convert from the Newby value to the new method value. If the $\mathrm{Fe}, \mathrm{Cr}, \mathrm{Pb}, \mathrm{Cl}, \mathrm{U}$ and $\mathrm{PO}_{4}$ in the feed are small, then the mole percent sodium plus potassium can be approximated from the Newby value by:

$$
\text { Molpcnt }_{\text {NaK }}=\frac{\text { Molpcnt }_{\text {Nax }}}{100+\text { Molpcnt }_{\text {NaK }}\left[\frac{1}{2} X_{N_{3}}\right]}(100 \%)
$$

Derivation of equation (6) is shown in Appendix D. For Newby's 5:1 'typical' Fluorinel/Sodium blend, the approximate value is in error by only +0.44 relative \%. Equation (6) should only be used to convert or compare previously reported mole\% $\mathrm{Na}+\mathrm{K}$ values to updated method values and should not be used for the NWCF feed blend calculations which are used for operational Run Plans.

The weight of calcined solids is also calculated for the 5:1 Fluorinel/Sodium blend used Newby's letter on mole percent calculation (Reference 2) and compared with the value calculated using the method in ENICO-1100 (Reference 1). For this feed blend, the value calculated using the new method (190.8 gm calcine per liter of feed) differs from the value calculated using the old method (216.7 gm calcine per liter of feed) by $13.6 \%$. The main reason for the difference is that, when a large excess of calcium ( $\mathrm{Ca} / \mathrm{F}$ ratio of 0.7 ) is used to suppress chloride volatility, the assumptions made in the old method that 1) $\mathrm{Na}_{3} \mathrm{AlF}_{6}$ and $\mathrm{K}_{3} \mathrm{AlF}_{6}$ form before $\mathrm{CaF}_{2}$ and 2) $50 \%$ of the excess calcium becomes nitrate, result in an overestimate of the amount of nitrates in the calcine.

For Newby's test case, the old method assumptions result in a calculated nitrate content of 19.5 $w t \%$ while the new method gives a nitrate content of $6.5 \mathrm{wt} \%$. By comparison, the nitrate content was 8.3 wt\% for calcine from Enclosed 30-cm Calciner Pilot Plant Run 10 (Reference 12), which calcined a Fluorinel/Sodium blend having a sodium plus potassium content similar to Newby's test case.

\section{EFFECTS OF NEGLECTING TRACE OR UNKNOWN CONCENTRATIONS OF FEED COMPONENTS}

Concentrations of minor feed components, such as phosphate, lead, and chromium, are often 
unavailable and must be assumed to be zero for the calculations. Neglecting trace metals such as lead and chromium, will reduce the moles of oxygen and total moles in the calcine, and will result in: 1) a conservative (higher) value for sodium plus potassium mole percent, 2) a lower weight of calcined solids, 3) a higher volume reduction factor, and 4) a conservatively higher heat generation rate. Neglecting phosphate will also provide: 1) a lower weight of calcined solids, 2) a higher volume reduction factor, and 3) a conservatively higher heat generation rate but will provide a negligibly lower (non-conservative) calculated mole $\% \mathrm{Na}+\mathrm{K}$.

In most cases the phosphate in the feed is unknown since it is difficult to analyze for in high nitric acid solutions. If phospate is neglected, calcium which would have formed $\mathrm{Ca}_{3}\left(\mathrm{PO}_{4}\right)_{2}$ would instead be assumed in the calculation to form $\mathrm{CaO}$, or every $2 / 3$ mole of phosphate would be replaced by 1 mole of oxide. Therefore, assuming the amount of phosphate is negligible would overestimate the total moles by $1 / 3$ of the actual phosphate concentration. This means the calculation would give a nonconservatively low sodium plus potassium value. The maximum phosphate concentration in the tank farm wastes is currently only $0.02 \underline{\mathrm{M}}$, and has never exceeded $0.028 \underline{\mathrm{M}}$ (Reference 14), so in general, assuming that the phosphate concentration is negligible will only introduce a very small nonconservative error $(0.1$ relative \%) to the calculated sodium plus potassium content. This is very small compared to the overall error for the calculations. In addition, this will be more than offset by the moles of other minor components such as cesium and strontium, which are neglected in the calculation.

\section{CALCULATIONAL ERROR}

The standard error associated with the calculated values (e.g. from sample analyses and solids bulk density data) is being estimated by J. J. Jacobson, and will be provided in a separate letter.

The population standard deviation has been determined by J. J. Jacobosen to be less than $8 \%$ $(\sigma<0.08$, References 16 and 17$)$ for heat generation rates calculated using the method described in this report for the volume reduction factor. The population standard deviation for the mole percent $\mathrm{Na}+\mathrm{K}$ calculated using the updated method in this report was determined to be 5.5 relative $\%(\sigma<0.055$, Reference 17). Factors for standard normal distribution should be used to determine confidence levels (' $z$ test') for determining confidence limits rather than ' $t$ test' values since Jacobsen used a very large 
population sample (10000 samples). For $95 \%$ confidence limits, the range is:

$$
X_{95 \% C . L}=\left(1 \pm 1.645 \frac{\sigma}{\sqrt{n}}\right) X_{n}
$$

where: $\sigma=$ population standard deviation, 0.08 for heat generation rate or 0.055 for mole percent sodium plus potassium

$\mathrm{X}_{\mathrm{n}}=$ Mean heat generation rate or mole percent sodium plus potassium for calcine for $\mathrm{n}$ samples

$\mathbf{n}=$ Number of data samples 


\section{REFERENCES}

1. K. F. Childs, R. I. Donovan, and M. C. Swenson, The Ninth Processing Campaign in the Waste Calcining Facility, ENICO-1100 (April 1982).

2. B. J. Newby, letter Nby-08-90 to R. P. Durante, "Calculation of Sodium Plus Potassium Mole Percent in Calcine from Blends of Fluorinel and Sodium Wastes," dated September 25, 1990.

3. K. N. Brewer, et. al., Physical and Chemical Characteristics of Fluorinel/Sodium Calcine Generated During $30 \mathrm{~cm}$ Pilot-plant Run 17, WINCO-1139 (July, 1993).

4. K. N. Brewer, personall communication, (January, 1993).

5. J. R. Berreth, Potential Calcining Stoichiometries and Their Effects on Waste Volumes, WINCO-1070 (December 1989).

6. "Facility for the Analysis of Chemical Thermodynamics-EQUILIB code," release date December 17, 1991, Mount-Royal,:Thermfact Ltd./Ltee.

7. K. J. Rebish, letter KJR-05-93 to B. H. O'Brien, "Multiple Linear Regression of Nitrate Versus Both Sodium Plus Potassium and Excess Calcium in Selected Pilot Plant Calcines," dated May 28, 1993.

8. B. R. Wheeler, E. S. Grimmett, and J. A. Buckham, Development of a Fluidized Bed Calcination Process for Aluminum Nitrate Wastes in a Two-foot-square Pilot Plant Calciner Part II: Factors Affecting the Intra-particle Porosity of Alumina, IDO-14587 (July 25, 1962).

9. B. H. Newby, T. D. Thomson, and B. H. O'Brien, Calcination of Fluorinel-Sodium Waste Blends Using Sugar as a Feed Additive, WINCO-1130 (June 1993).

10. R. I. Donovan, letter RID-08-89 to V. C. Maio, "1989 Tank Farm Inventory," dated September $14,1989$.

11. R. E. Schindler, Process Verification Testing for Burning TBP-containing Organic Solvents in the NWCF, ENICO-1086 (June 1981). 
12. B. H. O'Brien, letter BHO-09-89 to V. C. Maio, "Calciner Pilot Plant Database and Calcination Advisor," dated December 29, 1989.

13. Technical Standard 3.2E1, "Ca(NO3)2) Addition to Zirconium Fluoride Waste at NWCF," rev. 0, August 17, 1982.

14. K. J. Rebish, letter KJR-06-93 to J. M. Roberts, "Tank Farm Composition History," dated August 2, 1993.

15. A. K. Herbst, Mercury Concentratin in the Effluent Streams of the ICPP, ACI-378 (May 1979).

16. J. J. Jacobsen, letter JJJ-03-93 to B. H. O'Brien, "Error Analysis for Heat Generation Rate of Calcine," dated April 5, 1993.

17. J. J. Jacobsen, letter JJJ-01-95 to B. H. O'Brien, "Error Analysis for Heat Generation Rate of Calcine," dated March 9, 1995.

18. D. R. Wenzel, letter Wen-01-95 to R. J. Rivard, "Total Recoverable Energy Released During Radionuclide Decay", dated January 30, 1995.

19. R. J. Rivard, letter RJR-05-94 to B. H. O'Brien, "Maximum Expected Future Blend Concentrations for the NWCF", dated May 24, 1994.

20. R. J. Rivard, letter RJR-07-94 to B. H. O'Brien, "Update of Radiochemical Composition for NWCF Feeds of WM-188/WM-183/WM-189 Evaporated Waste", dated August 5, 1994.

21. K. J. Rebish and J. A. Nenni, letter KJR-06-93/JAN-03-94 to B. H. O'Brien and D. V. Croson, "Tank Farm Inventory - June, 1994," dated June 23, 1994.

22. T. J. Muller, letter TJM-03-93 to B. H. O'Brien, "Run Plan for Enclosed 10-cm Calciner Pilot Plant Runs SBW4-45 and SBW4-46," dated July 13, 1993. 
Appendix A

Sample Detailed Calculation of Sodium Plus Potassium Content and Weight of Calcined Solids 


\section{Appendix A \\ Sample Detailed Calculation of Sodium Plus Potassium Content and Weight of Calcined Solids}

Wanted: Mole percent of sodium plus potassium in the calcine and the weight of solid calcine produced per liter of feed based on the calciner feed solution composition.

Method: 1. Make assumptions of the compounds formed and preference order during calcination.

2. Calculate moles of each compound formed to determine the moles of species which will volatilize and the moles of compounds which contain oxygen.

3. Calculate moles of oxygen in oxides

4. Mole percent $\mathrm{Na}+\mathrm{K}=$ (Moles $\mathrm{Na}+\mathrm{K}) /($ oxygen in oxides +moles of non-volatile elements)

5. Weight of calcine $=$ weight of non-volatile elements from feed + weight of oxygen in oxides

\section{Given:}

Solution Content for 1 liter of Solution- Test Case is a theoretical Fluoride/Sodium Waste blend (used for the sample calculation):

$$
\begin{array}{llll}
\mathrm{F}:=2 \cdot \mathrm{mole} & \mathrm{Al}:=.5 \cdot \mathrm{mole} & \mathrm{Ca}:=1.4 \cdot \mathrm{mole} & \mathrm{Cr}:=0.0001 \cdot \mathrm{mole} \\
\mathrm{SO} 4:=0.1 \cdot \mathrm{mole} & \mathrm{Zr}:=0.25 \cdot \mathrm{mole} & \mathrm{B}:=0.15 \cdot \mathrm{mole} & \mathrm{Pb}:=0.0001 \cdot \mathrm{mole} \\
\mathrm{Cl}:=0.01 \cdot \mathrm{mole} & \mathrm{Na}:=0.25 \cdot \mathrm{mole} & \mathrm{Fe}:=0.1 \cdot \mathrm{mole} & \mathrm{U}:=0.0001 \cdot \mathrm{mole} \\
\mathrm{PO} 4:=0.001 \cdot \mathrm{mole} & \mathrm{K}:=0.05 \cdot \mathrm{mole} & \mathrm{Cd}:=0.1 \cdot \mathrm{mole} & \mathrm{UDS}:=1 \cdot \mathrm{gm}
\end{array}
$$

(Nitrate and acid concentrations in feed are not used in the calculation, but are given here for completeness)

NO3 $:=5 \cdot$ mole Acid $:=1.5 \cdot$ mole

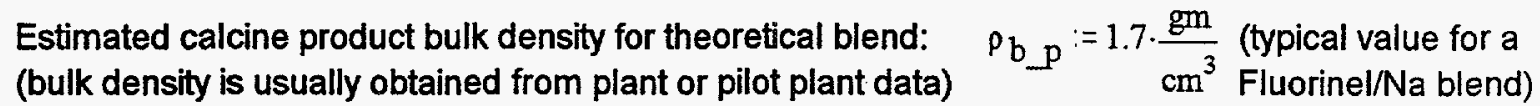

Molecular Weights and Atomic Weights (from the CRC Handbook of Chemistry and Physics, 62nd ed):

$$
\begin{aligned}
& \mathrm{W}_{\mathrm{Al}} \equiv 26.98154 \cdot \mathrm{gm} \cdot \mathrm{mole}^{-1} \quad \mathrm{~W}_{\mathrm{F}} \equiv 18.9984 \cdot \mathrm{gm} \cdot \mathrm{mole}^{-1} \quad \mathrm{~W}_{\mathrm{P}} \equiv 30.9738 \cdot \mathrm{gm} \cdot \mathrm{mole}^{-1} \\
& \mathrm{~W}_{\mathrm{B}} \equiv 10.81 \cdot \mathrm{gm} \cdot \mathrm{mole}^{-1} \quad \mathrm{~W}_{\mathrm{Fe}} \equiv 55.847 \cdot \mathrm{gm} \cdot \mathrm{mole}^{-1} \quad \mathrm{~W}_{\mathrm{Pb}} \equiv 207.19 \cdot \mathrm{gm} \cdot \mathrm{mole}{ }^{-1} \\
& \mathrm{~W}_{\mathrm{Ca}} \equiv 40.08 \cdot \mathrm{gm} \cdot \mathrm{mole}^{-1} \quad \mathrm{~W}_{\mathrm{K}} \equiv 39.0983 \cdot \mathrm{gm} \cdot \mathrm{mole}^{-1} \quad \mathrm{~W}_{\mathrm{S}} \equiv 32.064 \cdot \mathrm{gm} \cdot \mathrm{mole}^{-1} \\
& \mathrm{~W}_{\mathrm{Cd}} \equiv 112.41 \cdot \mathrm{gm} \cdot \mathrm{mole}^{-1} \quad \mathrm{~W}_{\mathrm{N}}=14.0067 \cdot \mathrm{gm} \cdot \mathrm{mole}^{-1} \quad \mathrm{~W}_{U} \equiv 235 \cdot \mathrm{gm} \cdot \mathrm{mole}^{-1} \\
& \mathrm{~W}_{\mathrm{Cl}} \equiv 35.453 \cdot \mathrm{gm} \cdot \mathrm{mole}^{-1} \quad \mathrm{~W}_{\mathrm{Na}} \equiv 22.98977 \cdot \mathrm{gm} \cdot \mathrm{mole}^{-1} \\
& \mathrm{~W}_{\mathrm{Cr}} \equiv 51.996 \cdot \mathrm{gm} \cdot \mathrm{mole}^{-1} \quad \mathrm{~W}_{\mathrm{O}} \equiv 15.9994 \cdot \mathrm{gm} \cdot \mathrm{mole}^{-1} \quad \mathrm{~W}_{\mathrm{Zr}} \equiv 91.22 \cdot \mathrm{gm} \cdot \mathrm{mole} \mathrm{e}^{-1} \\
& \begin{array}{ll}
\mathrm{w}_{\mathrm{NO} 3} \equiv\left(\mathrm{w}_{\mathrm{N}}+3 \cdot \mathrm{w}_{\mathrm{O}}\right) & \mathrm{w}_{\mathrm{NO} 3}=62.005 \cdot \mathrm{gm} \cdot \mathrm{mole}^{-1} \\
\mathrm{w}_{\mathrm{PO} 4} \equiv\left(\mathrm{w}_{\mathrm{P}}+4 \cdot \mathrm{w}_{\mathrm{O}}\right) & \mathrm{w}_{\mathrm{PO} 4}=94.971 \cdot \mathrm{gm} \cdot \mathrm{mole}^{-1} \\
\mathrm{w}_{\mathrm{SO} 4} \equiv\left(\mathrm{w}_{\mathrm{S}}+4 \cdot \mathrm{w}_{\mathrm{O}}\right) & \mathrm{w}_{\mathrm{SO} 4}=96.062 \cdot \mathrm{gm} \cdot \mathrm{mole}^{-1}
\end{array}
\end{aligned}
$$




\section{Assumptions:}

Compounds Assumed to be Formed During ICPP Waste Calcination:

Components $\rightarrow$ Products (Molecular Weight), listed in order of preference for formation

1. $\mathrm{NO}_{3}(62.067) \rightarrow \mathrm{NaNO}_{3}+\mathrm{KNO}_{3} \quad \ldots$ Nitrates are assumed proportional to $\mathrm{Na}+\mathrm{K}$

2. $\mathrm{PO}_{4}(94.971) \rightarrow \mathrm{Ca}_{3}\left(\mathrm{PO}_{4}\right)_{2}(310.18)+\mathrm{Na}_{3} \mathrm{PO}_{4}+\mathrm{K}_{3} \mathrm{PO}_{4}$

3. $\mathrm{F}(18.9984) \rightarrow \mathrm{CaF}_{2}(78.08)+\mathrm{Na}_{3} \mathrm{AlF}_{6}+\mathrm{KF}$

4. $\mathrm{SO}_{4}(96.058) \rightarrow \mathrm{K}_{2} \mathrm{SO}_{4}(174.27)+\mathrm{Na}_{2} \mathrm{SO}_{4}(142.04)+\mathrm{CaSO}_{4}(132.14)+\mathrm{CdSO}_{4}(208.47)$

5. $\mathrm{Cl}(35.453) \rightarrow \mathrm{KCl}(74.56)+\mathrm{NaCl}(58.443)+\mathrm{CaCl}_{2}(110.99)$

6. $\mathrm{Na}(22.98977) \rightarrow \mathrm{NaNO}_{3}(84.99)+\mathrm{Na}_{2} \mathrm{SO}_{4}(142.04)+\mathrm{NaCl}(58.443)+\mathrm{NaAlO}_{2}+\mathrm{Na}_{2} \mathrm{O}(81.97)^{*}$

7. $\mathrm{K}(39.0983) \rightarrow \mathrm{KNO}_{3}(101.11)+\mathrm{K}_{2} \mathrm{SO}_{4}(174.27)+\mathrm{KCl}(74.56)+\mathrm{KAIO}_{2}+\mathrm{K}_{2} \mathrm{O}(94.20)^{*}$

8. $\mathrm{Al}(26.98154) \rightarrow \mathrm{NaAlO}_{2}+\mathrm{KAlO}_{2}+\mathrm{Al}_{2} \mathrm{O}_{3}(101.96)$

9. $\mathrm{Ca}(40.08) \rightarrow \mathrm{Ca}_{3}\left(\mathrm{PO}_{4}\right)_{2}(310.18)+\mathrm{CaF}_{2}(78.08)+\mathrm{CaSO}_{4}(132.14)+\mathrm{CaCl}_{2}(110.99)$

$$
+\mathrm{CaO}(56.08)
$$

10. $\mathrm{Cd}(112.41) \rightarrow \mathrm{CdSO}_{4}(208.47)+\mathrm{CdO}(128.41)$

11. $\mathrm{Zr}(91.22) \rightarrow \mathrm{ZrO}_{2}(123.22)$

12. $\mathrm{B}(10.81) \rightarrow \mathrm{B}_{2} \mathrm{O}_{3}(69.92)$

13. $\mathrm{Fe}(55.847) \rightarrow \mathrm{Fe}_{2} \mathrm{O}_{3}(159.69)$

14. $\mathrm{Cr}(51.996) \rightarrow \mathrm{Cr}_{2} \mathrm{O}_{3}(151.99)$

15. $\mathrm{Pb}(207.19) \rightarrow \mathrm{PbO}(223.19)$

16. $U(235) \rightarrow \mathrm{UO}_{3}(283.00) \rightarrow$ use average uranium isotopic weight instead of 235 if known

17. Undissolved Solids,UDS $\rightarrow$ UDS weight added to weight of calcine solids

18. Other:

$\mathrm{Hg}(200.59) \rightarrow$ Assume most volatilizes and $\mathrm{HgO}$ remaining is negligible

$\mathrm{Ag}, \mathrm{As}, \mathrm{Sb}, \mathrm{Cs}, \mathrm{Ce}, \mathrm{Ni}, \mathrm{Mn}, \mathrm{Mo}, \mathrm{Nb}, \mathrm{Sn}, \mathrm{Gd} \ldots$... Assume negligible since concentrations in most tank farm wastes are $<0.5 \mathrm{~g} / \mathrm{L}$. If the concentration of a metal is known and is $>0.5 \mathrm{~g} / \mathrm{L}$ in the feed blend, then assume it forms an oxide of the same oxidation state as the metal in solution.

NOTES:

- $\mathrm{Na}$ and $\mathrm{K}$ oxides will exist primarily as mixed metal oxides with other metals such as $\mathrm{Al}$ and $\mathrm{B}$.

** Although they are usually less than $0.5 \mathrm{~g} / \mathrm{L}$ in calciner feed, the toxic metals cadmium, chromium, and lead, and also uranium are included in the calculation because their content in calcine is usually desired for other purposes. 


\section{Calculations:}

NOTES:

1. In equations that follow, equations along the left side with ":=" or " $\equiv "$ operators are equation definitions, while the values preceded by " $="$ along the right side are values calculated using the test case solution composition.

2. Syntax for "if" statements is: " $x:=i f(a \geq b, c, d)$ " is equivalent to "If $a$ is greater than or equal to $b$, then set $x$ equal to $c$, otherwise set $x$ equal to $d$."

1. Nitrate Solids:

Average Mole fraction of $\mathrm{Na}$ and $\mathrm{K}$ as nitrates in calcine based on analyses of calcine:

$$
\begin{array}{lc}
\mathrm{X}_{\mathrm{NO} 3}=0.66 \frac{\text { mole } \cdot \mathrm{NO}_{3}}{\text { mole } \cdot(\mathrm{Na}+\mathrm{K})} & \begin{array}{l}
(0.66 \pm 0.05 \text { based on analyses of pilot plant calcine without } \\
\text { reducing agents, Reference } 7 . \text { Use pilot plant calcine data } \\
\text { for } \left.\mathrm{NO}_{3} /(\mathrm{Na}+\mathrm{K}) \text { ratios if reducing agents such as sugar are used. }\right)
\end{array} \\
\mathrm{NaNO} 3:=\mathrm{X}_{\mathrm{NO} 3} \cdot \mathrm{Na} & \mathrm{NaNO} 3=0.165 \cdot \text { mole } \\
\mathrm{KNO} 3:=\mathrm{X}_{\mathrm{NO} 3} \cdot \mathrm{K} & \mathrm{KNO} 3=0.033 \cdot \mathrm{mole}
\end{array}
$$

\section{Phosphate solids:}

If enough $\mathrm{Ca}$ for $\mathrm{Phosphate,} \mathrm{then} \mathrm{all} \mathrm{phospate} \mathrm{forms} \mathrm{Ca}_{3}\left(\mathrm{PO}_{4}\right)_{2}$ :

$$
\mathrm{Ca} 3 \mathrm{PO}_{4} \_:=\text {if }\left(\mathrm{Ca} \geq \frac{3}{2} \cdot \mathrm{PO} 4, \frac{\mathrm{PO} 4}{2}, \frac{\mathrm{Ca}}{3}\right)
$$

Check value of inequality ( $=0$ for false, 1 for true):

$$
\begin{aligned}
& \left(\mathrm{Ca} \geq \frac{3}{2} \cdot \mathrm{PO} 4\right)=1 \\
& \mathrm{Ca} 3 \mathrm{PO} 4 \_2=5 \cdot 10^{-4} \cdot \mathrm{mole}
\end{aligned}
$$

If not enough $\mathrm{Ca}$ for $\mathrm{Ca}_{3}\left(\mathrm{PO}_{4}\right)_{2}$, then

If enough $\mathrm{Na}$ is available then remaining phospate forms $\mathrm{Na3PO} 4$ else all remaining $\mathrm{Na}$ forms $\mathrm{Na} 3 \mathrm{PO} 4$ :

$$
\begin{array}{ll}
\mathrm{PO}_{\text {rem }}:=\mathrm{PO} 4-2 \cdot \mathrm{Ca} 3 \mathrm{PO} 42_{2} & \mathrm{PO}_{\text {rem }}=0 \cdot \text { mole } \\
\mathrm{Na} 3 \mathrm{PO} 4:=\mathrm{if}\left[\mathrm{PO} 4_{\mathrm{rem}} \geq \frac{\left(1-\mathrm{X}_{\mathrm{NO} 3}\right) \cdot \mathrm{Na}}{3}, \frac{\left(1-\mathrm{X}_{\mathrm{NO} 3}\right) \cdot \mathrm{Na}}{3}, \mathrm{PO} 4_{\mathrm{rem}}\right] \mathrm{Na3PO} 4=0 \cdot \mathrm{mole}
\end{array}
$$

Check value of inequality ( $=0$ for false, 1 for true):

$$
\left[\mathrm{PO}_{\mathrm{rem}} \geq \frac{\left(1-\mathrm{X}_{\mathrm{NO} 3}\right) \cdot \mathrm{Na}}{3}\right]=0
$$




\section{Phosphate solids (cont.):}

If not enough $\mathrm{Na}$, then

If enough $\mathrm{K}$ is available then remaining phospate forms $\mathrm{K} 3 \mathrm{PO} 4$ else all remaining $\mathrm{K}$ forms $\mathrm{K} 3 \mathrm{PO}$ :

$$
\begin{aligned}
& \mathrm{PO}_{\text {rem_b }}:=\mathrm{PO} 4 \text { - 2.Ca3PO4_2 - Na3PO4 } \\
& \mathrm{PO} 4_{\text {rem_b }}=0 \text {-mole } \\
& \mathrm{K} 3 \mathrm{PO} 4:=\mathrm{if}\left[\mathrm{PO} \mathrm{rem} \_\mathrm{b} \geq \frac{\left(1-\mathrm{X}_{\mathrm{NO} 3}\right) \cdot \mathrm{K}}{3}, \frac{\left(1-\mathrm{X}_{\mathrm{NO} 3}\right) \cdot \mathrm{K}}{3}, \mathrm{PO}_{\mathrm{rem} \_\mathrm{b}}\right] \mathrm{K} 3 \mathrm{PO} 4=0 \cdot \mathrm{mole} \\
& \text { Check value of inequality ( }=0 \text { for false, } 1 \text { for true): } \\
& {\left[\mathrm{PO}_{\mathrm{rem} \_\mathrm{b}} \geq \frac{\left(1-\mathrm{X}_{\mathrm{NO} 3}\right) \cdot \mathrm{K}}{3}\right]=0}
\end{aligned}
$$

If not enough $\mathrm{Ca}, \mathrm{Na}$, or $\mathrm{K}$ available, assume remaining phosphate volatilizes:

$$
\mathrm{PO} 4_{\text {volat }}:=\mathrm{PO} 4-2 \cdot \mathrm{Ca} 3 \mathrm{PO} 42-\mathrm{Na3PO} 4-\mathrm{K} 3 \mathrm{PO} 4 \quad \mathrm{PO} 4 \text { volat }=0 \cdot \text { mole }
$$

\section{Fluoride Solids:}

If sufficient $\mathrm{Ca}$ after phosphate is formed, all $\mathrm{F}$ forms $\mathrm{CaF} 2$, else all remaining $\mathrm{Ca}$ forms $\mathrm{CaF2}$ :

$$
\begin{array}{cc}
\mathrm{Ca}_{\text {avail3 }}:=\mathrm{Ca}-3 \cdot \mathrm{Ca} 3 \mathrm{PO} 4 \_2 & \mathrm{Ca} \text { avail3 }=1.398 \cdot \mathrm{mole} \\
\mathrm{CaF2}:=\mathrm{if}\left(\frac{\mathrm{F}}{2} \geq \mathrm{Ca} \text { avail3 }, \mathrm{Ca} \text { avail3 }, \frac{\mathrm{F}}{2}\right) & \text { Check value of inequality }(=0 \text { for false } \\
\left(\frac{\mathrm{F}}{2} \geq \mathrm{Ca} \text { avail3 }\right)=0 \\
\mathrm{CaF} 2=1 \cdot \mathrm{mole}
\end{array}
$$

Check value of inequality ( $=0$ for false, 1 for true):

If not enough $\mathrm{Ca}$ available for $\mathrm{CaF} 2$, then

If enough $\mathrm{Na}$ is left after $\mathrm{NaNO} 3$ and $\mathrm{Na3PO} 4$ formation to form $\mathrm{NaF}$ then all remaining $\mathrm{F}$ forms $\mathrm{NaF}$ else all $\mathrm{Na}$ left forms $\mathrm{NaF}$ :

$$
\begin{array}{cc}
\mathrm{F}_{\text {rem }}:=\mathrm{F}-2 \cdot \mathrm{CaF} 2 & \mathrm{~F}_{\text {rem }}=0 \cdot \text { mole } \\
\mathrm{Na} & \mathrm{Na} \text { avail3 }:=\mathrm{Na}-\mathrm{NaNO} 3-3 \cdot \mathrm{Na} 3 \mathrm{PO} 4 \\
\mathrm{NaF}:=\mathrm{if}\left(\mathrm{F}_{\text {rem }} \geq \mathrm{Na} \text { avail3 }, \mathrm{Na} \text { avail3 }, \mathrm{F}_{\text {rem }}\right) & \text { Check value of inequality }(=0 \text { for false, } 1 \text { for true }): \\
\left(\mathrm{F}_{\text {rem }} \geq \mathrm{Na} \text { avail3 }\right)=0 \\
\mathrm{NaF}=0 \cdot \text { mole }
\end{array}
$$


3. Fluoride Solids (cont.):

If not enough $\mathrm{Ca}$ for $\mathrm{CaF} 2$ and not enough $\mathrm{Na}$ or $\mathrm{Al}$ for $\mathrm{NaF}$, then If enough $\mathrm{K}$ is available after $\mathrm{KNO} 3$ formation for $\mathrm{KF}$ then all remaining $F$ forms $\mathrm{KF}$ Else if not enough $\mathrm{K}$ not as $\mathrm{KNO} 3$ for $\mathrm{KF}$ then all remaining $\mathrm{K}$ forms $\mathrm{KF}$ :

$$
\begin{array}{ll}
\mathrm{F}_{\text {rem_b }}:=(\mathrm{F}-2 \cdot \mathrm{CaF} 2-\mathrm{NaF}) & \mathrm{F}_{\text {rem_b }}=0 \\
\mathrm{~K}_{\text {avail3 }}:=\mathrm{K}-\mathrm{KNO3}-3 \cdot \mathrm{K} 3 \mathrm{PO} 4 & \mathrm{~K}_{\text {avail3 }}=0.017 \cdot \mathrm{mole} \\
\mathrm{KF}:=\mathrm{if}\left(\mathrm{F}_{\text {rem_b }} \geq \mathrm{K}_{\text {avail3 }}, \mathrm{K}_{\text {avail3 }}, \mathrm{F}_{\text {rem_b }}\right) & \mathrm{KF}=0 \cdot \text { mole }
\end{array}
$$

Check value of inequality ( $=0$ for false, 1 for true):

$$
\left(F_{\text {rem_b }} \geq K_{\text {avail } 3}\right)=0
$$

Any remaining $\mathrm{F}$ not forming compounds with $\mathrm{Ca}, \mathrm{Na}$ and $\mathrm{K}$ is conservatively assumed to volatilize:

$$
F_{\text {volat }}:=F-2 \cdot \mathrm{CaF} 2-\mathrm{NaF}-\mathrm{KF} \quad \mathrm{F}_{\text {volat }}=0 \cdot \text { mole }
$$

\section{Sulfate (SO4) Solids:}

Potassium not as nitrate, phospate, or fluoride:

$$
\mathrm{K}_{\text {avail4 }}:=\mathrm{K}-\mathrm{KNO} 3-3 \cdot \mathrm{K} 3 \mathrm{PO} 4-\mathrm{KF} \quad \mathrm{K}_{\text {avail4 }}=0.017 \cdot \mathrm{mole}
$$

If sulfate is greater than remaining $\mathrm{K}$, then all remaining $\mathrm{K}$ forms $\mathrm{K} 2 \mathrm{SO} 4$, Otherwise, all sulfate forms K2SO4:

$$
\begin{aligned}
& \mathrm{K} 2 \mathrm{SO} 4:=\mathrm{if}\left(\mathrm{SO} 4 \geq \frac{\mathrm{K}_{\text {avail } 4}}{2}, \frac{\mathrm{K}_{\text {avail4 }}}{2}, \mathrm{SO}_{4}\right) \\
& \mathrm{K} 2 \mathrm{SO} 4=0.009 \cdot \mathrm{mole} \\
& \text { Check value of inequality ( }=0 \text { for false, } 1 \text { for true): } \\
& \left(\mathrm{SO} 4 \geq \frac{\mathrm{K} \text { avail }}{2}\right)=1 \\
& \mathrm{SO} 4_{\text {rem_a }}:=\mathrm{SO} 4-\mathrm{K} 2 \mathrm{SO} 4 \\
& \mathrm{SO}_{\text {rem_a }}=0.091 \cdot \mathrm{mole}
\end{aligned}
$$




\section{Sulfate (SO4) Solids (cont):}

If there is any sulfate remaining, it forms $\mathrm{Na2SO} 4$ if there is enough $\mathrm{Na}$ available.

Available $\mathrm{Na}$ not as nitrate, phosphate or fluoride:

$$
\mathrm{Na}_{\text {avail } 4}:=\mathrm{Na}-\mathrm{NaNO}-3 \cdot \mathrm{Na} 3 \mathrm{PO} 4-\mathrm{NaF} \quad \mathrm{Na} \text { avail4 }=0.085 \cdot \mathrm{mole}
$$

$$
\mathrm{Na} 2 \mathrm{SO} 4:=\text { if }\left(\mathrm{SO}_{\mathrm{rem}_{-} \mathrm{a}} \geq \frac{\mathrm{Na} \text { avail4 }}{2}, \frac{\mathrm{Na}_{\text {avail4 }}}{2}, \mathrm{SO}_{\text {rem_a }}\right) \quad \mathrm{Na} 2 \mathrm{SO} 4=0.042 \cdot \mathrm{mole}
$$

Check value of inequality ( $=0$ for false, 1 for true):

$$
\left(\mathrm{SO}_{\text {rem_a }} \geq \frac{\mathrm{Na} \text { avail4 }}{2}\right)=1
$$

$$
\mathrm{SO}_{\text {rem_b }}:=\mathrm{SO} 4-\mathrm{K} 2 \mathrm{SO} 4-\mathrm{Na} 2 \mathrm{SO} 4 \quad \mathrm{SO} 4_{\mathrm{rem} \_b}=0.049 \cdot \mathrm{mole}
$$

If there is any sulfate remaining, it forms CaSO4:

$$
\begin{array}{ll}
\mathrm{Ca}_{\text {avail4 }}:=\mathrm{Ca}-3 \cdot \mathrm{Ca} 3 \mathrm{PO} 4 \_2-\mathrm{CaF} 2 & \mathrm{Ca} \text { avail4 }=0.398 \cdot \mathrm{mole} \\
\mathrm{CaSO} 4:=\mathrm{if}\left(\mathrm{SO}_{4} \text { rem_b } \geq \mathrm{Ca}_{\text {avail4 }}, \mathrm{Ca} \text { avail4 }, \mathrm{SO}_{\text {rem_b }}\right) & \mathrm{CaSO} 4=0.049 \cdot \mathrm{mole}
\end{array}
$$

Check value of inequality ( $=0$ for false, 1 for true):

$\left(\mathrm{SO}_{\text {rem_b }} \geq \mathrm{Ca}\right.$ avail4 $)=0$

$$
\mathrm{SO} 4_{\text {rem_c }}:=\mathrm{SO} 4-\mathrm{K} 2 \mathrm{SO} 4-\mathrm{Na} 2 \mathrm{SO} 4-\mathrm{CaSO} 4 \quad \mathrm{SO}_{\mathrm{rem}_{-} \mathrm{c}}=0 \cdot \mathrm{mole}
$$

If there is any sulfate remaining, it forms $\mathrm{CdSO} 4$, otherwise it is conservatively assumed to volatilize:

$$
\begin{aligned}
& \mathrm{CdSO} 4:=\mathrm{if}\left(\mathrm{SO}_{\mathrm{rem} \_\mathrm{c}} \geq \mathrm{Cd}, \mathrm{Cd}, \mathrm{SO}_{\text {rem_c }}\right) \quad \mathrm{CdSO} 4=0 \cdot \mathrm{mole} \\
& \left(\mathrm{SO}_{\text {rem_c }} \mathrm{c} \geq \mathrm{Cd}\right)=0 \\
& \mathrm{SO}_{\text {volat }}:=\mathrm{SO} 4-\mathrm{K} 2 \mathrm{SO} 4-\mathrm{Na} 2 \mathrm{SO} 4-\mathrm{CaSO} 4-\mathrm{CdSO} 4 \quad \mathrm{SO} 4 \text { volat }=0 \cdot \text { mole }
\end{aligned}
$$

Check value of inequality ( $=0$ for false, 1 for true): 


\section{Chloride Solids:}

If enough $\mathrm{K}$, then form $\mathrm{KCl}$ :

$$
\begin{array}{ll}
\mathrm{K}_{\text {avail5 }}:=\mathrm{K}-\mathrm{KNO} 3-\mathrm{KF}-2 \cdot \mathrm{K} 2 \mathrm{SO} 4 & \mathrm{~K}_{\text {avail5 }}=0 \cdot \text { mole } \\
\mathrm{KCl}:=\mathrm{if}\left(\mathrm{Cl} \geq \mathrm{K}_{\text {avail5 }}, \mathrm{K}_{\text {avail5 }}, \mathrm{Cl}\right) & \mathrm{KCl}=0 \cdot \text { mole }
\end{array}
$$

Check value of inequality ( $=0$ for false, 1 for true):

$$
(\mathrm{Cl} \geq \mathrm{K} \text { avails })=1
$$

$$
\mathrm{Cl}_{\text {rem_a }}:=\mathrm{Cl}-\mathrm{KCl}
$$

$$
\mathrm{Cl}_{\text {rem_a }}=0.01 \cdot \mathrm{mole}
$$

Form $\mathrm{NaCl}$ if any $\mathrm{Cl}$ remains:

$$
\begin{array}{ll}
\mathrm{Na} \text { avail5 }:=\mathrm{Na}-\mathrm{NaNO} 3-\mathrm{NaF}-2 \cdot \mathrm{Na} 2 \mathrm{SO} 4 & \mathrm{Na} \text { avail5 }=0 \cdot \text { mole } \\
\mathrm{NaCl}:=\text { if }\left(\mathrm{Cl}_{\text {rem_a }} \geq \mathrm{Na} \text { avail5 }, \mathrm{Na} \text { avail5 }, \mathrm{Cl}_{\text {rem_a }}\right) & \mathrm{NaCl}=0 \cdot \text { mole }
\end{array}
$$

Check value of inequality ( $=0$ for false, 1 for true):

$$
\left(\mathrm{Cl}_{\text {rem_a }} \geq \mathrm{Na} \text { avail5 }\right)=1
$$

$$
\mathrm{Cl}_{\text {rem_b }}:=\mathrm{Cl}-\mathrm{KCl}-\mathrm{NaCl}
$$

$$
\mathrm{Cl}_{\text {rem_b }}=0.01 \cdot \mathrm{mole}
$$

Form $\mathrm{CaCl} 2$ if any $\mathrm{Cl}$ remains:

$$
\begin{array}{ll}
\mathrm{Ca} \text { avail5 }:=\mathrm{Ca}-3 \cdot \mathrm{Ca} 3 \mathrm{PO} 4 \_2-\mathrm{CaF} 2-\mathrm{CaSO} 4 & \mathrm{Ca} \text { avail5 }=0.349 \cdot \mathrm{mole} \\
\mathrm{CaCl} 2:=\text { if }\left(\frac{1}{2} \cdot \mathrm{Cl}_{\text {rem_b }} \geq \mathrm{Ca} \text { avail5 }, \mathrm{Ca} \text { avail5 }, \frac{\mathrm{Cl}_{\text {rem_b }}}{2}\right) & \mathrm{CaCl} 2=0.005 \cdot \mathrm{mole}
\end{array}
$$

Check value of inequality ( $=0$ for false, 1 for true):

Any remaining $\mathrm{Cl}$ volatilizes:

$$
\left(\mathrm{Cl}_{\text {rem_b }} \geq \frac{1}{2} \cdot \mathrm{Ca}_{\text {avails }}\right)=0
$$

$$
\mathrm{Cl}_{\text {volat }}:=\mathrm{Cl}-\mathrm{KCl}-\mathrm{NaCl}-2 \cdot \mathrm{CaCl} 2 \quad \mathrm{Cl}_{\text {volat }}=0 \cdot \text { mole }
$$




\section{Sodium Solids:}

Any remaining $\mathrm{Na}$ forms oxides which may or may not exist as complexes with other oxide compounds such as Aluminum oxide:

$$
\begin{array}{lc}
\mathrm{Na} \text { avail6 }:=\mathrm{Na}-\mathrm{NaNO}-2 \cdot \mathrm{Na} 2 \mathrm{SO} 4-\mathrm{NaCl}-\mathrm{NaF} \text { avail6 }=0 \cdot \text { mole } \\
\mathrm{NaAlO} 2:=\text { if }(\mathrm{Al}>\mathrm{Na} \text { avail6 }, \mathrm{Na} \text { avail6 }, \mathrm{Al}) & \text { Check value of inequality }(=0 \text { for false, } 1 \text { for true }): \\
(\mathrm{Al}>\mathrm{Na} \text { avail6 })=1 \\
\mathrm{Na} 2 \mathrm{NaAlO}=0 \cdot \mathrm{mole} \\
=\frac{1}{2} \cdot(\mathrm{Na} \text { avail6 }-\mathrm{NaAlO} 2) & \mathrm{Na} 2 \mathrm{O}=0 \cdot \text { mole }
\end{array}
$$

\section{Potassium Solids:}

Any remaining $\mathrm{K}$ forms oxides which may or may not exist as complexes with other oxide compounds such as Aluminum oxide:

$$
\begin{array}{lc}
\mathrm{K}_{\text {avail7 }}:=\mathrm{K}-\mathrm{KNO} 3-2 \cdot \mathrm{K} 2 \mathrm{SO} 4-\mathrm{KCl}-\mathrm{KF} & \mathrm{K}_{\text {avail } 7}=0 \cdot \text { mole } \\
\mathrm{KAlO} 2:=\mathrm{if}\left(\mathrm{Al}-\mathrm{NaAlO} 2>\mathrm{K}_{\text {avail }}, \mathrm{K}_{\text {avail7 }}, \mathrm{Al}-\mathrm{NaAlO} 2\right) \\
\text { Check value of inequality }(=0 \text { for false, } 1 \text { for true }): \\
\left(\mathrm{Al}-\mathrm{NaAlO} 2>\mathrm{K}_{\text {avail } 7}\right)=1 \\
\mathrm{KAlO} 2=0 \cdot \text { mole } \\
\mathrm{K} 20:=\frac{1}{2} \cdot\left(\mathrm{K}_{\text {avail7 }}-\mathrm{KAlO} 2\right) & \mathrm{K} 2 \mathrm{O}=0 \cdot \text { mole }
\end{array}
$$

\section{Aluminum Solids:}

Aluminum oxide:

$$
\text { Al2O3 }:=\frac{1}{2} \cdot(\mathrm{Al}-\mathrm{NaAlO} 2-\mathrm{KAlO} 2) \quad \mathrm{Al} 2 \mathrm{O} 3=0.25 \cdot \mathrm{mole}
$$

\section{Calcium Solids:}

Any remaining Calcium forms oxide:

$$
\mathrm{CaO}:=\mathrm{Ca}-3 \cdot \mathrm{Ca} 3 \mathrm{PO} 4 \_2-\mathrm{CaF} 2-\mathrm{CaSO} 4-\mathrm{CaCl} 2 \quad \mathrm{CaO}=0.344 \cdot \mathrm{mole}
$$

10. Cadmium Solids:

Any remaining Cadmium forms oxide:

$$
\mathrm{CdO}:=\mathrm{Cd}-\mathrm{CdSO} 4 \quad \mathrm{CdO}=0.1 \cdot \text { mole }
$$




\section{CALCULATE SODIUM PLUS POTASSIUM MOLE PERCENT}

Moles of oxygen in compounds with cations $\mathrm{Al}, \mathrm{Na}, \mathrm{K}, \mathrm{Ca}$ and $\mathrm{Cd}$ :

$$
\mathrm{O}_{\mathrm{a}}:=2 \cdot \mathrm{NaAlO} 2+2 \cdot \mathrm{KAlO} 2+\mathrm{Na} 2 \mathrm{O}+\mathrm{K} 2 \mathrm{O}+3 \cdot \mathrm{Al} 2 \mathrm{O} 3+\mathrm{CaO}+\mathrm{CdO} \quad \mathrm{O}_{\mathrm{a}}=1.194 \cdot \mathrm{mole}
$$

Moles of oxygen for feed components which are assumed to form only oxides:

$$
\begin{aligned}
& O_{b}:=2 \cdot \mathrm{Zr}+\frac{3}{2} \cdot \mathrm{B}+\frac{3}{2} \cdot \mathrm{Fe}+\frac{3}{2} \cdot \mathrm{Cr}+\mathrm{Pb}+3 \cdot \mathrm{U} \\
& \mathrm{O}_{\text {tot }}:=\mathrm{O}_{\mathrm{a}}+\mathrm{O}_{\mathrm{b}}
\end{aligned}
$$$$
\begin{aligned}
& \mathrm{O}_{\mathrm{b}}=0.876 \cdot \mathrm{mole} \\
& \mathrm{O}_{\text {tot }}=2.07 \cdot \mathrm{mole}
\end{aligned}
$$

Total Moles is sum of moles of oxygen + nitrate + feed components - volatilized components:

$$
\begin{aligned}
& \mathrm{Mol}_{\text {tot }}:=\mathrm{O}_{\text {tot }}+\mathrm{Al}+\left(1+\mathrm{X}_{\mathrm{NO} 3}\right) \cdot(\mathrm{Na}+\mathrm{K})+\mathrm{Ca} \ldots \quad \text { (Note that } \mathrm{X}_{\mathrm{NO}^{*}}(\mathrm{Na}+\mathrm{K}) \text { is the moles of nitrate) } \\
& +(\mathrm{PO} 4-\mathrm{PO} 4 \text { volat })+\left(\mathrm{F}-\mathrm{F}_{\text {volat }}\right) \ldots \\
& +(\mathrm{SO} 4-\mathrm{SO} 4 \text { volat })+\left(\mathrm{Cl}-\mathrm{Cl}_{\text {volat }}\right) \cdots \\
& +\mathrm{Zr}+\mathrm{B}+\mathrm{Fe}+\mathrm{Cd}+\mathrm{Cr}+\mathrm{Pb}+\mathrm{U} \\
& \mathrm{X}_{\mathrm{NO} 3}=0.66 \frac{\text { mole } \cdot \mathrm{NO}_{3}}{\mathrm{~mole} \cdot(\mathrm{Na}+\mathrm{K})} \\
& \mathrm{Mol}_{\text {tot }}=7.179 \cdot \mathrm{mole} \\
& \mathrm{Mol} \% \mathrm{NaK}:=\frac{\mathrm{Na}+\mathrm{K}}{\mathrm{Mol}_{\text {tot }}} \cdot 100 \% \\
& { }^{\mathrm{Mol}} \%{ }_{\mathrm{NaK}}=4.179 \cdot \%
\end{aligned}
$$

\section{CALCULATE WEIGHT OF CALCINED SOLIDS}

Total calcine weight is the sum of (MW x moles):

(Note: For Uranium, use average isotopic weight for uranium instead of 235 , or weight conc (in $\mathrm{g} / \mathrm{L}$ ) of uranium instead of "235 $\mathrm{U}$ ", if available )

$$
\begin{aligned}
\text { Calcine_Wt }_{\text {tot }}: & \mathrm{W}_{\mathrm{O}} \cdot \mathrm{O}_{\text {tot }}+\mathrm{W}_{\mathrm{Al}} \cdot \mathrm{Al}+\mathrm{W}_{\mathrm{NO}} \cdot \mathrm{X}_{\mathrm{NO} 3} \cdot(\mathrm{Na}+\mathrm{K}) \ldots \\
& +\mathrm{W}_{\mathrm{Na}} \cdot \mathrm{Na}+\mathrm{W}_{\mathrm{K}} \cdot \mathrm{K}+\mathrm{W}_{\mathrm{Ca}} \cdot \mathrm{Ca} \ldots \\
& +\mathrm{W}_{\mathrm{PO} 4} \cdot(\mathrm{PO} 4-\mathrm{PO} 4 \text { volat } \\
& +\mathrm{W}_{\mathrm{SO} 4} \cdot\left(\mathrm{W}_{\mathrm{F}} \cdot\left(\mathrm{F}-\mathrm{F}_{\text {volat }}\right) \ldots\right. \\
& +\mathrm{WO}_{\mathrm{Zr}} \cdot \mathrm{Zr}+\mathrm{W}_{\mathrm{B}} \cdot \mathrm{B}+\mathrm{W}_{\mathrm{Fe}} \cdot \mathrm{Fe}+\mathrm{W}_{\mathrm{Cd}} \cdot \mathrm{Cd}+\mathrm{W}_{\mathrm{Cr}} \cdot \mathrm{Cr}+\mathrm{W}_{\mathrm{Pb}} \cdot \mathrm{Pb} \ldots \\
& +\mathrm{W}_{\mathrm{U}} \cdot \mathrm{U}+\mathrm{UDS}
\end{aligned}
$$

Substituting for molecular/atomic weights:

$$
\begin{aligned}
\text { Calcine_Wt tot: }= & 15.9994 \cdot \mathrm{O}_{\text {tot }}+26.98154 \cdot \mathrm{Al}+62.005 \cdot \mathrm{X} \mathrm{NO}_{3} \cdot(\mathrm{Na}+\mathrm{K}) \ldots \\
& +22.98977 \cdot \mathrm{Na}+39.0983 \cdot \mathrm{K}+40.08 \cdot \mathrm{Ca} \ldots \\
& +94.971 \cdot(\mathrm{PO} 4-\mathrm{PO} 4 \text { volat })+18.9984 \cdot\left(\mathrm{F}-\mathrm{F}_{\text {volat }}\right) \ldots \\
& +96.062 \cdot(\mathrm{SO} 4-\mathrm{SO} 4 \text { volat })+35.453 \cdot\left(\mathrm{Cl}-\mathrm{Cl}_{\text {volat }}\right) \ldots \\
& +91.22 \cdot \mathrm{Zr}+10.81 \cdot \mathrm{B}+55.847 \cdot \mathrm{Fe}+112.41 \cdot \mathrm{Cd}+51.996 \cdot \mathrm{Cr} \ldots \\
& +207.19 \cdot \mathrm{Pb}+235 \cdot \mathrm{U}+\mathrm{UDS} \quad \quad \text { Calcine_Wt tot }=213.1 \cdot \mathrm{gm} \text { (per liter } \\
&
\end{aligned}
$$




\section{CALCULATE VOLUME REDUCTION FACTOR OF CALCINED SOLIDS}

(after Appendix G of ENICO-1100):

1. Estimate bulk density of calcined solids product and fines from plant or pilot plant data:

$$
\text { (value for a typical Fluorinel/ } \mathrm{Na} \text { blend is) } \quad \rho_{\mathrm{b} \_\mathrm{p}}=1.7 \cdot \frac{\mathrm{gm}}{\mathrm{cm}^{3}}
$$

2. Estimate density of product and fines mixture based on expected product to fines ratio using the method in ENICO-1100. Use $95 \%$ of product bulk density in most cases. Use $90 \%$ of product bulk density if $P / F$ ratio is less than 2 . Using a higher density will generally result in a higher, and more conservative, calculated heat generation rate for the calcine.

(P/F ratio for a $\mathrm{FI} / \mathrm{Na}$ blend is 3 to $4 \mathrm{~kg}$ product per $\mathrm{kg}$ fines)

so use $95 \%$ in this case: $\quad \rho_{b_{-} m}:=.95 \cdot \rho_{b} p_{0} \quad \rho_{b_{-} m}=1.615 \cdot \frac{\mathrm{gm}}{\mathrm{cm}^{3}}$

3. Volume reduction factor $=$ ratio of volume of liquid feed to volume of calcine solid produced:

$$
\text { VRF }:=\frac{\rho_{\mathrm{b}} \mathrm{m}^{\cdot} \cdot 1000 \cdot \frac{\mathrm{cm}^{3}}{\text { liter }^{2}}}{\text { Calcine_Wt } \mathrm{Wt}_{\text {tot }} \cdot \text { liter }^{-1}} \quad \text { VRF }=7.58 \frac{\text { liter_feed }}{\text { liter_calcine }} \text { a }
$$

4. The VRF used by operations is usually the the ratio of tank farm waste volume to calcine volume, and not the total volume of waste and feed additives such as aluminum nitrate, calcium nitrate and boric acid. This allows a direct calculation of bin volumes from tank farm depletion from the volume reduction factor.

The calcined solids calculation gave the amount of solids based on a solution volume of 1 liter):

$$
\mathrm{V}_{\text {total }}:=1 \cdot \text { liter }
$$

For Fluorine/ $\mathrm{Na}$ blends, only calcium nitrate is added. For simplicity in the example, assume solid calcium nitrate was added directly to the feed and did not increase the solution volume:

$$
V_{\text {feed_additives }}:=0 \cdot \text { liter }
$$

The volume of tank farm waste in the feed blend is then:

$$
\begin{aligned}
& V_{\text {tankfarm_waste }}:=V_{\text {total }}-V_{\text {feed_additives }} \\
& V_{\text {tankfarm_waste }}=1 \cdot \text { liter } \\
& V_{\text {RF }} \text { TF }:=V R F \cdot\left(\frac{V_{\text {total }}-V_{\text {feed_additives }}}{V_{\text {total }}}\right) \quad V_{T F}=7.58 \quad \frac{\text { liter_tank_farm_waste }}{\text { liter_calcine }}
\end{aligned}
$$


Appendix B

Verification of Simplified Equation for Moles of Oxygen 


\section{APPENDIX B \\ VERIFICATION OF SIMPLIFIED EQUATION FOR MOLES OF OXYGEN}

If no phosphate, fluoride, sulfate, or chloride volatilize, then it can be shown for all cases that the total moles of oxygen can be determined from the sum of oxidation states of the other elements in the feed:

$$
\text { moles_O tot }:=\frac{1}{2} \cdot\left(\sum_{1} \text { oxidation_states } \cdot \text { moles_of_element }\right)_{0} \quad \text { (Oxygen has a }-2 \text { oxidation state) }
$$

This works since any elements remaining after formation of phospates, fluorides, sulfates and chlorides are assumed to form oxides, and the oxidation states for all elements in the compounds formed must balance.

This method is less involved than that listed in Appendix A, since it does not require the calculation of the moles of each individual compound formed.

\section{DERIVATION OF SIMPLIFIED EQUATION FOR MOLES OF OXYGEN Example Case \#1-High Calcium and Potassium}

Wanted: Express oxygen moles in terms of elemental concentrations in feed by combining terms in equations for compounds formed.

Basis: 1 liter of solution

Method: 1. Determine equations for moles of each compound formed for the case given in terms of elemental/molar concentrations in feed.

2. Sum up moles of oxygen and substitute equations for oxides in terms of elemental compositions.

Given:

3. Collect terms to verify that moles of oxygen equals $0.5 \times$ (sum of (oxidation statesxelements)).

Case \#1-High calcium and potassium (enough calcium to react with phospate and fluoride, and enough potassium to react with sulfate and chloride) or:

$\mathrm{Ca} \geq \frac{3}{2} \cdot \mathrm{PO} 4 \mathrm{a}$ and $\mathrm{Ca}-\frac{3}{2} \cdot \mathrm{PO} 4 \geq \frac{\mathrm{F}}{2}$ and $\left(1-\mathrm{X}_{\mathrm{NO} 3}\right) \cdot \mathrm{K} \geq 2 \cdot \mathrm{SO} 4 \mathrm{~g}$ and $\left(1-\mathrm{X}_{\mathrm{NO} 3}\right) \cdot \mathrm{K}-2 \cdot \mathrm{K} 2 \mathrm{SO} 4 \geq \mathrm{Cl} \mathrm{o}$

Test Solution Content for 1 liter of Solution-Case \#1(High calcium and potassium):

$\begin{array}{llll}\mathrm{F}:=1 \cdot \mathrm{mole} & \mathrm{Al}:=1 \cdot \mathrm{mole} & \mathrm{Ca}:=.8 \cdot \mathrm{mole} & \mathrm{Cr}:=.0001 \cdot \mathrm{mole} \\ \mathrm{SO} 4:=.01 \cdot \mathrm{mole} & \mathrm{Zr}:=1 \cdot \mathrm{mole} & \mathrm{B}:=.1 \cdot \mathrm{mole} & \mathrm{Pb}:=.0001 \cdot \mathrm{mole} \\ \mathrm{Cl}:=.01 \cdot \mathrm{mole} & \mathrm{Na}:=.75 \cdot \mathrm{mole} & \mathrm{Fe}:=.1 \cdot \mathrm{mole} & \mathrm{Hg}:=.0001 \cdot \mathrm{mole} \\ \mathrm{PO} 4:=.001 \cdot \mathrm{mole} \mathrm{K}:=.4 \cdot \mathrm{mole} & \mathrm{Cd}:=.1 \cdot \mathrm{mole} & \mathrm{U}:=.000 \cdot \mathrm{mole} \\ \mathrm{NO} 3:=5 \cdot \mathrm{mole} & \text { Acid }:=2 \cdot \mathrm{mole} & \mathrm{UDS}:=1 \cdot \mathrm{gm} & \end{array}$




\section{Calculations:}

Verify there is enough $\mathrm{Ca}, \mathrm{Na}$, and $\mathrm{K}$, to react with all PO4, F, SO4, and $\mathrm{Cl}$ so that none of the PO4, $\mathrm{F}, \mathrm{SO} 4$, and $\mathrm{Cl}$ volatilize:

$$
\left[\left[2 \cdot \mathrm{Ca}+\left(1-\mathrm{X}_{\mathrm{NO} 3}\right) \cdot(\mathrm{Na}+\mathrm{K})\right] \geq(3 \cdot \mathrm{PO} 4+\mathrm{F}+2 \cdot \mathrm{SO} 4+\mathrm{Cl})\right]=1 \quad(=0 \text { for false, } 1 \text { for true })
$$

$\therefore$ True, therefore, there is sufficient $\mathrm{Ca}, \mathrm{Na}$, and $\mathrm{K}$ for all non-oxides assumed to form.

1. Nitrate Solids:

Average Mole fraction of $\mathrm{Na}$ and $\mathrm{K}$ as nitrates based on analysis of calcine:

$$
\begin{aligned}
& \mathrm{X}_{\mathrm{NO} 3}=0.66 \frac{\mathrm{mole}^{\mathrm{NO}} 3}{\mathrm{~mole} \cdot(\mathrm{Na}+\mathrm{K})} \quad(0.66 \pm 0.05 \text { based on analyses of pilot plant calcine, Reference } 7) \\
& \mathrm{NaNO}:=\mathrm{X}_{\mathrm{NO} 3} \cdot \mathrm{Na} \\
& \mathrm{KNO} 3:=\mathrm{X}_{\mathrm{NO} 3} \cdot \mathrm{K}
\end{aligned}
$$

2. Phosphate solids:

All goes to Calcium Phosphate since there is enough calcium:

$$
\begin{aligned}
& \mathrm{Ca} \geq \frac{3}{2} \cdot \mathrm{PO} 4=1 \quad(=0 \text { for false, } 1 \text { for true }) \\
& \text { Ca3PO4_2 }:=\frac{\mathrm{PO} 4}{2}
\end{aligned}
$$

No phospate to combine with $\mathrm{Na}$ and $\mathrm{K}$ so: $\mathrm{Na} 3 \mathrm{PO} 4:=0 \cdot$ mole K3PO4 :=0 mole

Check to make sure none volatilizes:

$$
\mathrm{PO} 4_{\text {volat }}:=\mathrm{PO} 4-2 \cdot \mathrm{Ca}_{\mathrm{POO}}{ }_{2} 2 \quad \mathrm{PO} 4 \text { volat }=0
$$

3. Fluoride Solids:

Since there is sufficient $\mathrm{Ca}$ not as phosphate, all F goes to CaF2:

$$
\begin{aligned}
& \mathrm{Ca}_{\text {rem }}:=\mathrm{Ca}-\frac{3}{2} \cdot \mathrm{PO} 4 \\
& \mathrm{Ca}_{\text {rem }} \geq \frac{\mathrm{F}}{2}=1 \quad(=0 \text { for false, } 1 \text { for true }) \\
& \mathrm{CaF} 2:=\frac{\mathrm{F}}{2} \quad \text { All } \mathrm{F} \text { goes to CaF } 2 \\
& \mathrm{NaF}:=0 \cdot \mathrm{mole}
\end{aligned}
$$

Check to make sure none volatilizes:

$$
F_{\text {volat }}:=\mathrm{F}-2 \cdot \mathrm{CaF} 2-\mathrm{NaF}-\mathrm{KF} \quad \mathrm{F}_{\text {volat }}=0
$$




\section{Sulfate (SO4) Solids:}

Potassium not as nitrate or fluoride:

$$
\mathrm{K}_{\mathrm{rem} 4}:=\mathrm{K}-\mathrm{X}_{\mathrm{NO} 3} \cdot \mathrm{K}-\mathrm{KF}
$$

If sulfate is greater than remaining $\mathrm{K}$, then all remaining $\mathrm{K}$ goes to sulfate, Else all sulfate goes to $\mathrm{K} 2 \mathrm{SO} 4:$

$$
\begin{aligned}
& \mathrm{SO} 4 \geq \frac{\mathrm{K}_{\mathrm{rem} 4}}{2}=0 \quad(=0 \text { for false, so sulfate is not greater than remaining } \mathrm{K}): \\
& \mathrm{K} 2 \mathrm{SO} 4:=\mathrm{SO} 4 \quad \text { All SO4 goes to } \mathrm{K} 2 \mathrm{SO} 4 \\
& \mathrm{SO} 4 \mathrm{rem}:=\mathrm{SO} 4-\mathrm{K} 2 \mathrm{SO} 4 \\
& \mathrm{SO}_{4} \mathrm{rem}=0 \cdot \mathrm{mole} \\
& \mathrm{Na} 2 \mathrm{SO} 4:=0 \cdot \mathrm{mole} \\
& \mathrm{CaSO} 4:-0 \cdot \mathrm{mole} \\
& \mathrm{CdSO} 4:=0 \cdot \text { mole }
\end{aligned}
$$

Check to make sure none volatilizes:

$$
\begin{aligned}
& \mathrm{SO} 4 \text { volat }:=\mathrm{SO} 4-\mathrm{K} 2 \mathrm{SO} 4-\mathrm{Na} 2 \mathrm{SO} 4-\mathrm{CaSO} 4-\mathrm{CdSO} 4 \\
& \mathrm{SO}_{\text {volat }}=0
\end{aligned}
$$

\section{Chloride Solids:}

If enough $\mathrm{K}$, then form $\mathrm{KCl}$ :

$$
\begin{aligned}
& \mathrm{K}_{\text {rem5 }}:=\mathrm{K}-\mathrm{X}_{\mathrm{NO} 3} \cdot \mathrm{K}-\mathrm{KF}-2 \cdot \mathrm{K} 2 \mathrm{SO} 4 \\
& \left.\mathrm{Cl} \geq \mathrm{K}_{\text {rem5 }}=0 \text { (=0 for false, so } \mathrm{Cl} \text { is not greater than remaining } \mathrm{K}\right) \text { : } \\
& \mathrm{KCl}:=\mathrm{Cl} \\
& \mathrm{Cl}_{\text {rem }}:=\mathrm{Cl}-\mathrm{KCl} \\
& \mathrm{Cl}_{\text {rem }}=0 \\
& \mathrm{NaCl}:=0 \cdot \text { mole } \\
& \mathrm{CaCl} 2:=0 \cdot \text { mole }
\end{aligned}
$$

Check to make sure none volatilizes:

$$
\begin{aligned}
& \mathrm{Cl}_{\text {volat }}:=\mathrm{Cl}-\mathrm{KCl}-\mathrm{NaCl}-2 \cdot \mathrm{CaCl} 2 \\
& \mathrm{Cl}_{\text {volat }}=0
\end{aligned}
$$




\section{Sodium Solids:}

Any remaining $\mathrm{Na}$ forms oxides which may or may not exist as complexes with other oxide compounds such as Aluminum oxide:

$$
\begin{aligned}
& \mathrm{Na}_{\text {avail6 }}:=\mathrm{Na}-\mathrm{NaNO} 3-3 \cdot \mathrm{Na} 3 \mathrm{PO} 4-2 \cdot \mathrm{Na} 2 \mathrm{SO} 4-\mathrm{NaCl}-\mathrm{NaF} \\
& \text { and } \mathrm{NaF}=0 \quad \mathrm{Na} 3 \mathrm{PO} 4=0 \quad \mathrm{Na} 2 \mathrm{SO} 4=0 \quad \mathrm{NaCl}=0 \\
& \text { so: } \quad \mathrm{Na} \text { avail6 }:=(\mathrm{Na}-\mathrm{NaNO} 3)
\end{aligned}
$$

Substituting for NaNO3:

$$
\begin{aligned}
& \mathrm{Na} \text { avail6 }:=\left(\mathrm{Na}-\mathrm{X}_{\mathrm{NO} 3} \cdot \mathrm{Na}\right) \\
& \mathrm{NaAlO} 2:=\text { if }(\mathrm{Al}>\mathrm{Na} \text { avail6 }, \mathrm{Na} \text { avail6 }, \mathrm{Al}) \quad \text { and } \quad\left(\mathrm{Al}>\mathrm{Na}_{\text {avail6 }}\right)=1 \text { so: } \\
& \mathrm{NaAlO} 2:=\mathrm{Na} \text { avail6 or: } \quad \mathrm{NaAlO} 2:=\left(\mathrm{Na}-\mathrm{X}_{\mathrm{NO} 3} \cdot \mathrm{Na}\right) \\
& \mathrm{Na2O}:=\frac{1}{2} \cdot\left(\mathrm{Na}_{\text {avail6 }}-\mathrm{NaAlO}\right) \quad \text { but there is no } \mathrm{Na} \text { left so: } \mathrm{Na} 2 \mathrm{O}=0 \cdot \text { mole }
\end{aligned}
$$

\section{Potassium Solids:}

Any remaining $\mathrm{K}$ forms oxides which may or may not exist as complexes with other oxide compounds such as Aluminum oxide:

$$
\begin{aligned}
& \mathrm{K}_{\text {avail7 }}:=\mathrm{K}-\mathrm{KNO} 3-3 \cdot \mathrm{K} 3 \mathrm{PO} 4-2 \cdot \mathrm{K} 2 \mathrm{SO} 4-\mathrm{KCl}-\mathrm{KF} \\
& \text { and } \mathrm{KF}=0 \quad \mathrm{~K} 3 \mathrm{PO} 4=0 \\
& \text { so: } \mathrm{K}_{\text {avail7 }}:=\mathrm{K}-\mathrm{KNO} 3-2 \cdot \mathrm{K} 2 \mathrm{SO} 4-\mathrm{KCl}
\end{aligned}
$$

Substituting for $\mathrm{KNO} 3, \mathrm{~K} 2 \mathrm{SO}$, and $\mathrm{KCl}$ :

$$
\mathrm{K}_{\text {avail7 }}:=\mathrm{K}-\mathrm{X}_{\mathrm{NO} 3} \cdot \mathrm{K}-2 \cdot \mathrm{SO} 4-\mathrm{Cl}
$$

$$
\mathrm{KAlO} 2:=\mathrm{if}\left(\mathrm{Al}-\mathrm{NaAlO} 2>\mathrm{K}_{\text {avail7 }}, \mathrm{K}_{\text {avail7 }}, \mathrm{Al}\right) \text { and } \quad\left(\mathrm{Al}-\mathrm{NaAlO} 2>\mathrm{K}_{\text {avail7 }}\right)=1
$$

so there is enough $\mathrm{Al}$ for all $\mathrm{K}$ and: $\mathrm{KAlO} 2:=\mathrm{K}_{\text {avail }}$

substituting gives: $\mathrm{KAlO} 2:=\mathrm{K}-\mathrm{X}_{\mathrm{NO} 3} \cdot \mathrm{K}-2 \cdot \mathrm{SO} 4-\mathrm{Cl}$

$$
\mathrm{K} 2 \mathrm{O}:=\frac{1}{2} \cdot\left(\mathrm{K}_{\text {avail } 7}-\mathrm{KAlO} 2\right) \quad \text { so: } \quad \mathrm{K} 2 \mathrm{O}=0 \cdot \text { mole }
$$

\section{Aluminum Solids (other than $\mathrm{NaAlO} 2$ and KAIO2):}

Aluminum oxide:

$$
\mathrm{Al} 2 \mathrm{O} 3:=\frac{1}{2} \cdot(\mathrm{Al}-\mathrm{NaAlO} 2-\mathrm{KAlO} 2)
$$

Substituting for $\mathrm{NaAlO} 2$ and $\mathrm{KAIO} 2$ gives:

$$
\mathrm{Al} 2 \mathrm{O} 3:=\frac{1}{2} \cdot\left[\mathrm{Al}-\left(\mathrm{Na}-\mathrm{X}_{\mathrm{NO} 3} \cdot \mathrm{Na}\right)-\left(\mathrm{K}-\mathrm{X}_{\mathrm{NO} 3} \cdot \mathrm{K}-2 \cdot \mathrm{SO} 4-\mathrm{Cl}\right)\right]
$$




\section{Calcium Solids:}

Any remaining Calcium goes to oxide:

$$
\begin{aligned}
& \mathrm{CaO}:=\mathrm{Ca}-3 \cdot \mathrm{Ca} 3 \mathrm{PO} 4 \_2-\mathrm{CaF} 2-\mathrm{CaSO} 4-\mathrm{CaCl} 2 \\
& \mathrm{CaSO} 4=0 \text { and } \mathrm{CaCl} 2=0 \text { so: } \\
& \mathrm{CaO}:=\mathrm{Ca}-3 \cdot \mathrm{Ca} 3 \mathrm{PO} 4 \_2-\mathrm{CaF} 2
\end{aligned}
$$

Substituting for Ca3PO_2 and CaF2

$$
\mathrm{CaO}:=\mathrm{Ca}-\frac{3}{2} \cdot \mathrm{PO} 4-\frac{1}{2} \cdot \mathrm{F}
$$

10. Cadmium Solids:

Any remaining Cadmium goes to oxide:

$$
\begin{aligned}
& \mathrm{CdO}:=\mathrm{Cd}-\mathrm{CdSO} 4 \text { and } \mathrm{CdSO} 4:=0 \text { so: } \\
& \mathrm{CdO}:=\mathrm{Cd}
\end{aligned}
$$




\section{CALCULATE MOLES OF OXYGEN}

Moles of oxygen in compounds with $\mathrm{Al}, \mathrm{Na}, \mathrm{K}$, and $\mathrm{Ca}$ :

$$
\mathrm{O}_{\mathrm{a}}:=2 \cdot \mathrm{NaAlO} 2+2 \cdot \mathrm{KAlO} 2+\mathrm{Na} 2 \mathrm{O}+\mathrm{K} 2 \mathrm{O}+3 \cdot \mathrm{Al} 2 \mathrm{O} 3+\mathrm{CaO}+\mathrm{CdO}
$$

Substituting for moles of $\mathrm{NaAlO} 2$ and $\mathrm{KAlO} 2, \mathrm{Na2O}$ and $\mathrm{K} 2 \mathrm{O}, \mathrm{Al} 2 \mathrm{O} 3, \mathrm{CaO}, \mathrm{CdO}$ :

$$
\begin{aligned}
\mathrm{O}_{\mathrm{a}}:= & 2 \cdot\left(\left(\mathrm{Na}-\mathrm{X}_{\mathrm{NO} 3} \cdot \mathrm{Na}\right)\right)+2 \cdot\left(\mathrm{K}-\mathrm{X}_{\mathrm{NO} 3} \cdot \mathrm{K}-2 \cdot \mathrm{SO} 4-\mathrm{Cl}\right) \ldots \\
& +0 \cdot \mathrm{mole}+0 \cdot \mathrm{mole}_{\ldots} \ldots \\
& +3 \cdot\left[\frac{1}{2} \cdot\left[\mathrm{Al}-\left(\mathrm{Na}-\mathrm{X}_{\mathrm{NO} 3} \cdot \mathrm{Na}\right)-\left(\mathrm{K}-\mathrm{X}_{\mathrm{NO} 3} \cdot \mathrm{K}-2 \cdot \mathrm{SO} 4-\mathrm{Cl}\right)\right]\right] \ldots \\
& +\left(\mathrm{Ca}-\frac{3}{2} \cdot \mathrm{PO} 4-\frac{1}{2} \cdot \mathrm{F}\right) \ldots \\
& +\mathrm{Cd}
\end{aligned}
$$

simplifies to

$$
\mathrm{O}_{\mathrm{a}}:=\frac{1}{2} \cdot \mathrm{Na}-\frac{1}{2} \cdot \mathrm{X}_{\mathrm{NO} 3} \cdot \mathrm{Na}+\frac{1}{2} \cdot \mathrm{K}-\frac{1}{2} \cdot \mathrm{X} \mathrm{NO}_{3} \cdot \mathrm{K}-\mathrm{SO} 4-\frac{1}{2} \cdot \mathrm{Cl}+\frac{3}{2} \cdot \mathrm{Al}+\mathrm{Ca}-\frac{3}{2} \cdot \mathrm{PO} 4-\frac{1}{2} \cdot \mathrm{F}+\mathrm{Cd}
$$

rearranges to

$$
\mathrm{O}_{\mathrm{a}}:=\frac{1}{2} \cdot\left(3 \cdot \mathrm{Al}+\mathrm{Na}+\mathrm{K}+2 \cdot \mathrm{Ca}-\mathrm{X}_{\mathrm{NO} 3} \cdot \mathrm{Na}-\mathrm{X}_{\mathrm{NO} 3} \cdot \mathrm{K}-2 \cdot \mathrm{SO} 4-\mathrm{Cl}-3 \cdot \mathrm{PO} 4-\mathrm{F}+2 \cdot \mathrm{Cd}\right)
$$

or, collecting terms for $\mathrm{Na}$ and $\mathrm{K}$ :

$$
\mathrm{O}_{\mathrm{a}}:=\frac{1}{2} \cdot\left[3 \cdot \mathrm{Al}+\left(1-\mathrm{X}_{\mathrm{NO} 3}\right) \cdot \mathrm{Na}+\left(1-\mathrm{X}_{\mathrm{NO} 3}\right) \cdot \mathrm{K}+2 \cdot \mathrm{Ca}+-2 \cdot \mathrm{SO} 4+-1 \cdot \mathrm{Cl}+-3 \cdot \mathrm{PO} 4+-1 \cdot \mathrm{F}+2 \cdot \mathrm{Cd}\right]
$$

which can be represented as

$$
\mathrm{O}_{\mathrm{a}}:=\frac{1}{2} \cdot\left[\sum_{\text {elements }}((\text { oxidation_state }) \cdot(\text { moles_of_element }))\right] \mathrm{a}
$$

This is also true for elements assumed to form only oxides:

$$
\mathrm{O}_{\mathrm{b}}:=2 \cdot \mathrm{Zr}+\frac{3}{2} \cdot \mathrm{B}+\frac{3}{2} \cdot \mathrm{Fe}+\frac{3}{2} \cdot \mathrm{Cr}+\mathrm{Pb}+3 \cdot \mathrm{U}
$$

rearranges to

$$
\mathrm{O}_{\mathrm{b}}:=\frac{1}{2} \cdot(4 \cdot \mathrm{Zr}+3 \cdot \mathrm{B}+3 \cdot \mathrm{Fe}+3 \cdot \mathrm{Cr}+2 \cdot \mathrm{Pb}+6 \cdot \mathrm{U})
$$

Which is also the based on the sum of oxidation times concentration:

$$
\mathrm{O}_{\mathrm{b}}:=\frac{1}{2} \cdot\left[\sum_{\text {elements }}((\text { oxidation_state }) \cdot(\text { moles_of_element }))\right]
$$

$\therefore$ Therefore, the moles of oxygen the calcine can be determined from the sum of oxidation states of the other elements in the feed for this example. 


\section{Derive Simplified Equation for Moles of Oxygen \\ Example Case \#2 - High Calcium, But Not Enough Potassium for Sulfate}

Wanted: Express moles of oxygen in calcine in terms of elements in feed by combining terms in equations for compounds formed.

Basis: 1 liter of solution

Method: 1. Determine equations for moles of each compound formed for the case given in terms of elemental/molar concentrations in feed.

2. Sum up moles of oxygen and substitute equations for oxides in terms of elemental compositions.

3. Collect terms to verify that moles of oxygen $=0.5 \times$ (sum of (oxidation states $\times$ elements)).

\section{Given:}

Case \#2 - Not enough potassium to form sulfate, enough $\mathrm{Ca}$ and $\mathrm{Na}$ for remainder.

Basis: 1 liter of solution

Test Case Solution Content for 1 liter of Solution-Case \#2 (Not enough potassium to form sulfate, but enough $\mathrm{Ca}$ and $\mathrm{Na}$ for remainder)

$\begin{array}{llll}\mathrm{F}:=1 \cdot \mathrm{mole} & \mathrm{Al}:=1 \cdot \mathrm{mole} & \mathrm{Ca}:=.8 \cdot \mathrm{mole} & \mathrm{Cr}:=.0001 \cdot \mathrm{mole} \\ \mathrm{SO} 4:=.1 \cdot \mathrm{mole} & \mathrm{Zr}:=1 \cdot \mathrm{mole} & \mathrm{B}:=.1 \cdot \mathrm{mole} & \mathrm{Pb}:=.0001 \cdot \mathrm{mole} \\ \mathrm{Cl}:=.01 \cdot \mathrm{mole} & \mathrm{Na}:=.5 \cdot \mathrm{mole} & \mathrm{Fe}:=.1 \cdot \mathrm{mole} & \mathrm{Hg}:=.0001 \cdot \mathrm{mole} \\ \mathrm{PO} 4:=.001 \cdot \mathrm{mole} \mathrm{K}:=.1 \cdot \mathrm{mole} & \mathrm{Cd}:=.1 \cdot \mathrm{mole} & \mathrm{U}:=.000 \cdot \mathrm{mole} \\ \mathrm{NO} 3:=5 \cdot \mathrm{mole} & \text { Acid } \\ \mathrm{Na} & :=2 \cdot \mathrm{mole} & \mathrm{UDS}:=1 \cdot \mathrm{gm} & \end{array}$

\section{Calculations:}

Verify there is enough $\mathrm{Ca}, \mathrm{Na}$, and $\mathrm{K}$, to react with all $\mathrm{PO}$, F, SO4, and $\mathrm{Cl}$ so that none of the PO4, $\mathrm{F}$, SO4, and $\mathrm{Cl}$ volatilize:

$$
\left[\left[2 \cdot \mathrm{Ca}+\left(1-\mathrm{X}_{\mathrm{NO} 3}\right) \cdot(\mathrm{Na}+\mathrm{K})\right] \geq(3 \cdot \mathrm{PO} 4+\mathrm{F}+2 \cdot \mathrm{SO} 4+\mathrm{Cl})\right]=1 \quad(=0 \text { for false, } 1 \text { for true })
$$

$\therefore$ True, therefore, there is sufficient $\mathrm{Ca}, \mathrm{Na}$, and $\mathrm{K}$ for all non-oxides assumed to form.

(This is somewhat conservative since it doesn't account for possible CdSO4) 
1. Nitrate Solids:

Average Mole fraction of $\mathrm{Na}$ and $\mathrm{K}$ as nitrates based on analysis of calcine:

$$
\begin{aligned}
& \mathrm{X}_{\mathrm{NO} 3}:=0.66 \frac{\text { mole } \cdot \mathrm{NO}_{3}}{\text { mole } \cdot(\mathrm{Na}+\mathrm{K})} \\
& \mathrm{NaNO} 3:=\mathrm{X}_{\mathrm{NO} 3} \cdot \mathrm{Na} \\
& \mathrm{KNO} 3:=\mathrm{X}_{\mathrm{NO} 3} \cdot \mathrm{K}
\end{aligned}
$$

\section{Phosphate solids:}

All goes to Calcium Phosphate,

If not enough $\mathrm{Ca}$ for Phosphate, then excess phospate volatilizes:

$$
\begin{aligned}
& \mathrm{Ca} \geq \frac{3}{2} \cdot \mathrm{PO} 4=1 \quad(=1 \text { for true, so there is enough calcium }) \\
& \mathrm{Ca3PO} 42:=\frac{\mathrm{PO} 4}{2}
\end{aligned}
$$

No phospate to combine with $\mathrm{Na}$ and $\mathrm{K}$ so: $\mathrm{Na3PO} 4:=0 \cdot$ mole $\mathrm{K} 3 \mathrm{PO} 4:=0 \cdot \mathrm{mole}$ Check to make sure none volatilizes:

$$
\begin{aligned}
& \mathrm{PO} 4_{\text {volat }}:=\mathrm{PO} 4-2 \cdot \mathrm{Ca} 3 \mathrm{PO} 4 \text { 2 } 2-\mathrm{Na3PO} 4-\mathrm{K} 3 \mathrm{PO} 4 \\
& \mathrm{PO} 4 \text { volat }=0
\end{aligned}
$$

3. Fluoride Solids:

If sufficient $\mathrm{Ca}$ not as phosphate, all $\mathrm{F}$ goes to $\mathrm{CaF} 2$, else all $\mathrm{Ca}$ goes to $\mathrm{CaF2}$ :

$$
\begin{aligned}
& \mathrm{Ca}_{\text {avail3 }}:=\mathrm{Ca}-3 \cdot \mathrm{Ca} 3 \mathrm{PO} 422 \\
& \frac{\mathrm{F}}{2} \geq \mathrm{Ca}_{\text {avail3 } 3}=0 \quad \rightarrow=0 \text { for false, so all } \mathrm{F} \text { goes to } \mathrm{CaF} 2 \\
& \mathrm{CaF} 2:=\frac{\mathrm{F}}{2} \\
& \mathrm{~F}_{\text {rem }}:=\mathrm{F}-2 \cdot \mathrm{CaF} 2 \quad \mathrm{~F}_{\text {rem }}=0
\end{aligned}
$$

No flouride left to combine with $\mathrm{Na}$ and $\mathrm{K}$ so:

$$
\mathrm{NaF}:=0 \cdot \text { mole } \quad \mathrm{KF}:=0 \cdot \text { mole }
$$

Check to make sure none volatilizes:

$$
F_{\text {volat }}:=\mathrm{F}-2 \cdot \mathrm{CaF} 2-\mathrm{NaF}-\mathrm{KF} \quad \mathrm{F}_{\text {volat }}=0
$$




\section{Sulfate (SO4) Solids:}

Potassium not as nitrate or fluoride:

$$
\begin{aligned}
& \mathrm{K}_{\text {avail4 }}:=\mathrm{K}-\mathrm{X}_{\mathrm{NO} 3} \cdot \mathrm{K}-3 \cdot \mathrm{K} 3 \mathrm{PO} 4-\mathrm{KF} \\
& \mathrm{SO} 4 \geq \frac{\mathrm{K}_{\text {avail4 }}}{2}=1 \quad \rightarrow \text { Not enough } \mathrm{K} \text { to tie up all sulfate } \\
& \mathrm{K} 2 \mathrm{SO} 4:=\frac{\mathrm{K}_{\text {avail4 }}}{2} \quad \rightarrow \text { All available } \mathrm{K} \text { goes to } \mathrm{K} 2 \mathrm{SO} 4
\end{aligned}
$$

by substitution, yields

$$
\mathrm{K} 2 \mathrm{SO} 4:=\frac{1}{2} \cdot \mathrm{K}-\frac{1}{2} \cdot \mathrm{X}_{\mathrm{NO} 3} \cdot \mathrm{K}-\frac{1}{2} \cdot 0 \cdot \mathrm{mole}-\frac{1}{2} \cdot(3 \cdot 0 \cdot \mathrm{mole})
$$

simplifies to

$$
\begin{aligned}
& \mathrm{K} 2 \mathrm{SO} 4:=\frac{1}{2} \cdot \mathrm{K}-\frac{1}{2} \cdot \mathrm{X}_{\mathrm{NO} 3} \cdot \mathrm{K} \\
& \mathrm{SO}_{\text {rem_a }}:=\mathrm{SO} 4-\mathrm{K} 2 \mathrm{SO} 4
\end{aligned}
$$

If there is any sulfate left, it forms $\mathrm{Na}_{2} \mathrm{SO} 4, \mathrm{CaSO} 4$, and $\mathrm{CdSO} 4$ $\mathrm{Na}$ not as nitrate or fluoride:

$$
\begin{aligned}
& \mathrm{Na}_{\text {avail4 } 4}:=\mathrm{Na}-\mathrm{X}_{\mathrm{NO} 3} \cdot \mathrm{Na}-3 \cdot \mathrm{Na} 3 \mathrm{PO} 4-\mathrm{NaF} \\
& \mathrm{SO}_{\text {rem_a }} \geq \frac{\mathrm{Na} \text { avail4 }}{2}=0 \quad \rightarrow \text { All remaining } \mathrm{SO} 4 \text { goes to } \mathrm{Na} 2 \mathrm{SO} 4 \\
& \mathrm{Na} 2 \mathrm{SO} 4:=\mathrm{SO} 4 \text { rem_a }
\end{aligned}
$$

by substitution yields,

$$
\mathrm{Na} 2 \mathrm{SO} 4:=\mathrm{SO} 4-\mathrm{K} 2 \mathrm{SO} 4 \text { or } \quad \mathrm{Na} 2 \mathrm{SO} 4:=\mathrm{SO} 4-\left(\frac{1}{2} \cdot \mathrm{K}-\frac{1}{2} \cdot \mathrm{X}_{\mathrm{NO} 3} \cdot \mathrm{K}\right)
$$

No sulfate left for $\mathrm{Ca}$ and $\mathrm{Cd}$ :

$$
\begin{array}{ll}
\mathrm{SO}_{\text {rem_b }}:=\mathrm{SO} 4-\mathrm{K} 2 \mathrm{SO} 4-\mathrm{Na} 2 \mathrm{SO} 4 & \mathrm{SO}_{\text {rem_b }}=0 \\
\mathrm{CaSO} 4:=\mathrm{SO} 4 \text { rem_b } & \mathrm{CaSO} 4=0 \\
\mathrm{SO} 4 \text { rem_c }:=\mathrm{SO} 4-\mathrm{K} 2 \mathrm{SO} 4-\mathrm{Na} 2 \mathrm{SO} 4-\mathrm{CaSO} 4 & \mathrm{SO} 4{ }_{\text {rem_c }}=0 \\
\mathrm{CdSO} 4:=\mathrm{SO} 4 \text { rem_c } & \mathrm{CdSO} 4=0
\end{array}
$$

Verify nothing volatilizes

$$
\begin{aligned}
& \mathrm{SO}_{\text {volat }}:=\mathrm{SO} 4-\mathrm{K} 2 \mathrm{SO} 4-\mathrm{Na} 2 \mathrm{SO} 4-\mathrm{CaSO} 4-\mathrm{CdSO} 4 \\
& \mathrm{SO}_{\text {volat }}=0
\end{aligned}
$$




\section{Chloride Solids:}

If enough $\mathrm{K}$, then form $\mathrm{KCl}$ :

$\mathrm{K}_{\text {avail5 }}:=\mathrm{K}-\mathrm{X}_{\mathrm{NO} 3} \cdot \mathrm{K}-3 \cdot \mathrm{K} 3 \mathrm{PO} 4-\mathrm{KF}-2 \cdot \mathrm{K} 2 \mathrm{SO} 4 \quad \mathrm{~K}_{\text {avail5 }}=0$

$\mathrm{KCl}:=\mathrm{K}_{\text {avail5 }} \quad \mathrm{KCl}=0$

Else form $\mathrm{NaCl}$ :

$\mathrm{Cl}_{\text {rem_a }}:=\mathrm{Cl}-\mathrm{KCl}$

$\mathrm{Cl}_{\text {rem_a }}:=\mathrm{Cl}$

$\mathrm{Na}$ avail5 $:=\mathrm{Na}-\mathrm{X}_{\mathrm{NO} 3} \cdot \mathrm{Na}-3 \cdot \mathrm{Na} 3 \mathrm{PO} 4-\mathrm{NaF}-2 \cdot \mathrm{Na} 2 \mathrm{SO} 4$

$\mathrm{Cl}_{\text {rem_a }} \geq \mathrm{Na}$ avail5 $=1 \rightarrow$ Enough $\mathrm{Na}$, so all $\mathrm{Cl}$ goes to $\mathrm{NaCl}$

$$
\begin{aligned}
& \mathrm{NaCl}:=\mathrm{Cl}_{\text {rem_a }} \\
& \mathrm{NaCl}:=\mathrm{Cl}
\end{aligned}
$$

Else form $\mathrm{CaCl}$ :

$$
\begin{array}{ll}
\mathrm{Cl}_{\text {rem_b }}:=\mathrm{Cl}-\mathrm{KCl}-\mathrm{NaCl} & \mathrm{Cl}_{\text {rem_b }}=0 \\
\mathrm{CaCl} 2:=\frac{\mathrm{Cl}_{\text {rem_b }}}{2} & \mathrm{CaCl} 2=0
\end{array}
$$

Check to make sure no $\mathrm{Cl}$ volatilizes:

$$
\begin{aligned}
& \mathrm{Cl}_{\text {volat }}:=\mathrm{Cl}-\mathrm{KCl}-\mathrm{NaCl}-2 \cdot \mathrm{CaCl} 2 \\
& \mathrm{Cl}_{\text {volat }}=0
\end{aligned}
$$




\section{Sodium Solids:}

Any remaining $\mathrm{Na}$ forms oxides which may or may not exist as complexes with other oxide compounds such as Aluminum oxide:

$$
\begin{aligned}
& \mathrm{Na} \text { avail6 }:=(\mathrm{Na}-\mathrm{NaNO} 3-3 \cdot \mathrm{Na} 3 \mathrm{PO} 4-2 \cdot \mathrm{Na} 2 \mathrm{SO} 4-\mathrm{NaCl}-\mathrm{NaF}) \\
& \mathrm{NaF}=0 \text { and } \mathrm{Na} 3 \mathrm{PO} 4=0 \quad \text { so: } \\
& \mathrm{Na} \text { avail6 }:=(\mathrm{Na}-\mathrm{NaNO} 3-2 \cdot \mathrm{Na} 2 \mathrm{SO} 4-\mathrm{NaCl})
\end{aligned}
$$

Substituting for $\mathrm{NaNO} 3, \mathrm{Na} 2 \mathrm{SO} 4$, and $\mathrm{NaCl}$ :

$$
\begin{aligned}
& \mathrm{Na} \text { avail6 }:=\left[\mathrm{Na}-\mathrm{X}_{\mathrm{NO} 3} \cdot \mathrm{Na}-2 \cdot\left[\mathrm{SO} 4-\left(\frac{1}{2} \cdot \mathrm{K}-\frac{1}{2} \cdot \mathrm{X}_{\mathrm{NO} 3} \cdot \mathrm{K}\right)\right]-\mathrm{Cl}\right] \\
& \mathrm{NaAlO} 2:=\mathrm{if}(\mathrm{Al}>\mathrm{Na} \text { avail6}, \mathrm{Na} \text { avail6}, \mathrm{Al}) \quad \text { and } \quad(\mathrm{Al}>\mathrm{Na} \text { avail6 })=1 \quad \text { so: } \\
& \mathrm{NaAlO} 2:=\mathrm{Na} \text { avail6 } \quad \text { or: } \mathrm{NaAlO} 2:=\left[\mathrm{Na}-\mathrm{X}_{\mathrm{NO} 3} \cdot \mathrm{Na}-2 \cdot\left[\mathrm{SO} 4-\left(\frac{1}{2} \cdot \mathrm{K}-\frac{1}{2} \cdot \mathrm{X}_{\mathrm{NO} 3} \cdot \mathrm{K}\right)\right]-\mathrm{Cl}\right] \\
& \mathrm{Na} 2 \mathrm{O}:=\frac{1}{2} \cdot(\mathrm{Na} \text { avail6 }-\mathrm{NaAlO} 2) \quad \text { leaving } \quad \mathrm{Na} 2 \mathrm{O}=0 \cdot \text { mole }
\end{aligned}
$$

\section{Potassium Solids:}

Any remaining $\mathrm{K}$ forms oxides which may or may not exist as complexes with other oxide compounds such as Aluminum oxide:

$$
\begin{aligned}
& \mathrm{K}_{\text {avail } 7}:=(\mathrm{K}-\mathrm{KNO} 3-3 \cdot \mathrm{K} 3 \mathrm{PO} 4-2 \cdot \mathrm{K} 2 \mathrm{SO} 4-\mathrm{KCl}-\mathrm{KF}) \\
& \mathrm{K}_{\text {avail } 7}=0 \cdot \text { mole } \quad \text { no potassium left } \\
& \mathrm{KAlO} 2:=\mathrm{if}\left(\mathrm{Al}-\mathrm{NaAlO} 2>\mathrm{K}_{\text {avail7 }}, \mathrm{K}_{\text {avail }}, \mathrm{Al}\right) \text { and } \quad(\mathrm{Al}-\mathrm{NaAlO} 2>\mathrm{K} \text { avail7 })=1
\end{aligned}
$$

$$
\mathrm{K} 2 \mathrm{O}:=\frac{1}{2} \cdot\left(\mathrm{K}_{\text {avail } 7}-\mathrm{KAlO} 2\right) \quad \text { so: } \quad \mathrm{K} 2 \mathrm{O}=0 \cdot \text { mole }
$$

\section{Aluminum Solids (other than $\mathrm{NaAlO2}$ and $\mathrm{KAIO2}$ ):}

Aluminum oxide:

$$
\mathrm{Al} 2 \mathrm{O} 3:=\frac{1}{2} \cdot(\mathrm{Al}-\mathrm{NaAlO} 2-\mathrm{KAlO} 2)
$$

Substituting for $\mathrm{NaAlO} 2$ and $\mathrm{KAlO} 2$ gives:

$$
\mathrm{Al} 2 \mathrm{O} 3:=\frac{1}{2} \cdot\left[\mathrm{Al}-\left[\mathrm{Na}-\mathrm{X}_{\mathrm{NO} 3} \cdot \mathrm{Na}-2 \cdot\left[\mathrm{SO} 4-\left(\frac{1}{2} \cdot \mathrm{K}-\frac{1}{2} \cdot \mathrm{X}_{\mathrm{NO} 3} \cdot \mathrm{K}\right)\right]-\mathrm{Cl}\right]\right]
$$


9. Calcium Solids:

Any remaining Calcium goes to oxides:

$\mathrm{CaO}:=\mathrm{Ca}-$ 3.Ca3PO4_2 - $\mathrm{CaF} 2-\mathrm{CaSO} 4-\mathrm{CaCl} 2$

by substitution for Ca3PO4_2, CaF2, CaSO4, and $\mathrm{CaCl} 2$, yields

$$
\mathrm{CaO}:=\mathrm{Ca}-\frac{3}{2} \cdot \mathrm{PO} 4-\frac{1}{2} \cdot \mathrm{F}
$$

simplifies to

$$
\mathrm{CaO}:=\mathrm{Ca}-\frac{3}{2} \cdot \mathrm{PO} 4-\frac{1}{2} \cdot \mathrm{F}
$$

10. Cadmium Solids:

Any remaining Cadmium goes to oxide:

$$
\begin{array}{ll}
\mathrm{CdO}:=\mathrm{Cd}-\mathrm{CdSO} 4 & \mathrm{CdSO} 4=0 \\
\mathrm{CdO}:=\mathrm{Cd} &
\end{array}
$$




\section{CALCULATE SODIUM PLUS POTASSIUM MOLE PERCENT}

Moles of oxygen in compounds with $\mathrm{Al}, \mathrm{Na}, \mathrm{K}$, and $\mathrm{Ca}$ :

$$
\mathrm{O}_{\mathrm{a}}:=2 \cdot \mathrm{NaAlO} 2+2 \cdot \mathrm{KAlO} 2+\mathrm{Na} 2 \mathrm{O}+\mathrm{K} 2 \mathrm{O}+3 \cdot \mathrm{Al} 2 \mathrm{O} 3+\mathrm{CaO}+\mathrm{CdO}
$$

Substituting for moles of $\mathrm{NaAlO} 2$ and $\mathrm{KAlO} 2, \mathrm{Na} 2 \mathrm{O}$ and $\mathrm{K} 2 \mathrm{O}, \mathrm{Al} 2 \mathrm{O} 3, \mathrm{CaO}, \mathrm{CdO}$ :

$$
\begin{aligned}
\mathrm{O}_{\mathrm{a}}:= & 2 \cdot\left[\mathrm{Na}-\mathrm{X}_{\mathrm{NO} 3} \cdot \mathrm{Na}-2 \cdot\left[\mathrm{SO} 4-\left(\frac{1}{2} \cdot \mathrm{K}-\frac{1}{2} \cdot \mathrm{X}_{\mathrm{NO} 3} \cdot \mathrm{K}\right)\right]-\mathrm{Cl}\right] \ldots \\
& +3 \cdot\left[\frac{1}{2} \cdot\left[\mathrm{Al}-\left[\mathrm{Na}-\mathrm{X}_{\mathrm{NO} 3} \cdot \mathrm{Na}-2 \cdot\left[\mathrm{SO} 4-\left(\frac{1}{2} \cdot \mathrm{K}-\frac{1}{2} \cdot \mathrm{X}_{\mathrm{NO} 3} \cdot \mathrm{K}\right)\right]-\mathrm{Cl}\right]\right]\right] \ldots \\
& +\mathrm{Ca}-\frac{3}{2} \cdot \mathrm{PO} 4-\frac{1}{2} \cdot \mathrm{F}+\mathrm{Cd}
\end{aligned}
$$

simplifies to

$$
\mathrm{O}_{\mathrm{a}}:=\frac{1}{2} \cdot \mathrm{Na}-\frac{1}{2} \cdot \mathrm{X}_{\mathrm{NO} 3} \cdot \mathrm{Na}+\frac{1}{2} \cdot \mathrm{K}-\frac{1}{2} \cdot \mathrm{X}_{\mathrm{NO} 3} \cdot \mathrm{K}-\mathrm{SO} 4-\frac{1}{2} \cdot \mathrm{Cl}+\frac{3}{2} \cdot \mathrm{Al}+\mathrm{Ca}-\frac{3}{2} \cdot \mathrm{PO} 4-\frac{1}{2} \cdot \mathrm{F}+\mathrm{Cd}
$$

rearranges to

$$
\mathrm{O}_{\mathrm{a}}:=\frac{1}{2} \cdot\left[3 \cdot \mathrm{Al}+\left(1-\mathrm{X}_{\mathrm{NO} 3}\right) \cdot \mathrm{Na}+\left(1-\mathrm{X}_{\mathrm{NO} 3}\right) \cdot \mathrm{K}+2 \cdot \mathrm{Ca}-2 \cdot \mathrm{SO} 4-\mathrm{Cl}-3 \cdot \mathrm{PO} 4-\mathrm{F}+2 \cdot \mathrm{Cd}\right]
$$

which can be represented as

$$
\mathrm{O}_{\mathrm{a}}:=\frac{1}{2} \cdot\left[\sum_{\text {elements }}((\text { oxidation_state }) \cdot(\text { moles_of_element }))\right]
$$

This is also true for elements assumed to form only oxides:

$$
\mathrm{O}_{\mathrm{b}}:=2 \cdot \mathrm{Zr}+\frac{3}{2} \cdot \mathrm{B}+\frac{3}{2} \cdot \mathrm{Fe}+\frac{3}{2} \cdot \mathrm{Cr}+\mathrm{Pb}+3 \cdot \mathrm{U}
$$

rearranges to

$$
\mathrm{O}_{\mathrm{b}}:=\frac{1}{2} \cdot(4 \cdot \mathrm{Zr}+3 \cdot \mathrm{B}+3 \cdot \mathrm{Fe}+3 \cdot \mathrm{Cr}+2 \cdot \mathrm{Pb}+6 \cdot \mathrm{U})
$$

Which is also the based on the sum of oxidation times concentration

$$
\mathrm{O}_{\mathrm{b}}:=\frac{1}{2} \cdot\left[\sum_{\text {elements }}((\text { oxidation_state }) \cdot(\text { moles_of_element }))\right]
$$

$\therefore$ Therefore, the moles of oxygen the calcine can be determined from the sum of oxidation states of the other elements in the feed for this example. 
Appendix C

Example Calculations

C-1 


\section{EXAMPLE CALCULATION \#1}

\section{CASE Nby\#1}

Test Case Solution Content for 1 liter of Solution-Case Nby1 (Values from letter Nby-08-90, case 1):

Solution is a 5:1 blend of Typical Fluorinel and $\mathrm{Na}$ waste.

$$
\begin{aligned}
& \mathrm{F}:=\frac{5 \cdot 2.27+0.049}{6} \cdot \mathrm{mole} \quad \mathrm{Al}:=\frac{5 \cdot 0.36+.45}{6} \cdot \mathrm{mole} \quad \mathrm{Ca}:=0.7 \cdot \mathrm{F} \quad \mathrm{Cr}:=.0 \cdot \mathrm{mole} \\
& \mathrm{SO} 4:=\frac{5 \cdot 0.079+.048}{6} \text { mole } \mathrm{Zr}:=\frac{5 \cdot 0.29+0}{6} \cdot \text { mole } \quad \mathrm{B}:=\frac{5 \cdot 0.17+0.024}{6} \cdot \text { mole } \quad \mathrm{Pb}:=0 \cdot \text { mole } \\
& \mathrm{Cl}:=\frac{5 \cdot 0.0016+0.031}{6} \cdot \text { mole } \mathrm{Na}:=\frac{5 \cdot 0.028+1.46}{6} \cdot \text { mole } \mathrm{Fe}:=\frac{5 \cdot 0.0072+0.023}{6} \cdot \text { mole } \mathrm{U}:=0 \cdot \text { mole } \\
& \mathrm{PO}:=.0 \cdot \mathrm{mole} \quad \mathrm{K}:=\frac{5 \cdot 0.0028+.21}{6} \cdot \mathrm{mole} \quad \mathrm{Cd}: \square \frac{5 \cdot 0.11+0}{6} \cdot \text { mole } \begin{array}{l}
\text { (Use ave isotopic weight for } \\
\text { uranium if analyses is available) }
\end{array} \\
& \mathrm{NO} 3:=\left(\frac{5 \cdot 2.37+4.47}{6}\right) \cdot \text { mole }^{2}+\mathrm{Ca} \quad \text { Acid }_{\mathrm{Na}}:=\frac{5 \cdot 2.04+1.69}{6} \cdot \mathrm{mole} \quad \text { UDS }:=\frac{5 \cdot 2+1}{6} \cdot \mathrm{gm}
\end{aligned}
$$

Calculated concentrations for $5: 1$ blend:

$$
\begin{array}{llll}
\mathrm{F}=1.9 \cdot \mathrm{mole} & \mathrm{Al}=0.375 \cdot \mathrm{mole} & \mathrm{Ca}=1.33 \cdot \mathrm{mole} & \mathrm{Cr}=0 \cdot \mathrm{mole} \\
\mathrm{SO} 4=0.074 \cdot \mathrm{mole} & \mathrm{Zr}=0.242 \cdot \mathrm{mole} & \mathrm{B}=0.146 \cdot \mathrm{mole} & \mathrm{Pb}=0 \cdot \mathrm{mole} \\
\mathrm{Cl}=0.006 \cdot \mathrm{mole} & \mathrm{Na}=0.267 \cdot \mathrm{mole} & \mathrm{Fe}=0.01 & \mathrm{U}=0 \cdot \mathrm{mole} \\
\mathrm{PO} 4=0 \cdot \mathrm{mole} & \mathrm{K}=0.037 \cdot \mathrm{mole} & \mathrm{Cd}=0.092 \cdot \mathrm{mole} & \mathrm{UDS}=1.833 \cdot \mathrm{gm}
\end{array}
$$

(Nitrate and acid concentrations in feed are not used in the calculation, but are given here for completeness)

$\mathrm{NO} 3=5.38 \cdot \mathrm{mole} \quad \mathrm{Na}+\mathrm{K}=0.304 \cdot$ mole $\quad$ Acid $_{\mathrm{Na}}=1.982 \cdot \mathrm{mole}$

Estimated calcine product bulk density for Case \#Nby1: $\quad \rho_{b \_}:=1.7 \cdot \frac{\mathrm{gm}}{\mathrm{cm}^{3}}$ (value for a typical

Average Mole fraction of $\mathrm{Na}$ and $\mathrm{K}$ as nitrates based on analysis of calcine:

$$
\mathrm{X}_{\mathrm{NO} 3}:=0.66 \frac{\text { mole } \cdot \mathrm{NO}_{3}}{\text { mole }(\mathrm{Na}+\mathrm{K})} \quad \begin{aligned}
& (0.66 \pm 0.05 \text { based on analyses of pilot plant calcine without } \\
& \text { reducing agents, Reference } 7 . \text { Use pilot plant calcine data } \\
& \text { for } \mathrm{NO}_{3} /(\mathrm{Na}+\mathrm{K}) \text { ratios if reducing agents such as sugar are used.) }
\end{aligned}
$$

\section{Calculations:}

1. Verify there is enough $\mathrm{Ca}, \mathrm{Na}$, and $\mathrm{K}$, to react with all $\mathrm{PO} 4, \mathrm{~F}, \mathrm{SO}$, and $\mathrm{Cl}$ so that none of the $\mathrm{PO} 4, \mathrm{~F}, \mathrm{SO} 4$, and $\mathrm{Cl}$ volatilize:

$$
\left[\left[2 \cdot \mathrm{Ca}+\left(1-\mathrm{X}_{\mathrm{NO} 3}\right) \cdot(\mathrm{Na}+\mathrm{K})\right] \geq(3 \cdot \mathrm{PO} 4+\mathrm{F}+2 \cdot \mathrm{SO} 4+\mathrm{Cl})\right]=1 \quad(=0 \text { for false, } 1 \text { for true })
$$

$\therefore$ True, therefore, there is sufficient $\mathrm{Ca}, \mathrm{Na}$, and $\mathrm{K}$ for all non-oxides assumed to form.

(This is somewhat conservative since it doesn't account for possible CdSO4) 
2. Calculate moles of oxygen:

There is enough $\mathrm{Ca}, \mathrm{Na}, \mathrm{K}$, and $\mathrm{Al}$, so the simplified equation for determining moles of oxygen is preferred to the detailed calculation of Appendix A:

Total moles of oxygen in oxides is therefore:

$$
\begin{aligned}
\mathrm{O}_{\text {tot }}:= & \frac{3}{2} \cdot \mathrm{Al}+\frac{1}{2} \cdot\left(1-\mathrm{X}_{\mathrm{NO} 3}\right) \cdot \mathrm{Na}+\frac{1}{2} \cdot\left(1-\mathrm{X}_{\mathrm{NO} 3}\right) \cdot \mathrm{K}+\mathrm{Ca}-\mathrm{SO} 4-\frac{1}{2} \cdot \mathrm{Cl}-\frac{3}{2} \cdot \mathrm{PO} 4-\frac{1}{2} \cdot \mathrm{F}+\mathrm{Cd} \ldots \\
& +2 \cdot \mathrm{Zr}+\frac{3}{2} \cdot \mathrm{B}+\frac{3}{2} \cdot \mathrm{Fe}+\frac{3}{2} \cdot \mathrm{Cr}+\mathrm{Pb}+3 \cdot \mathrm{U} \quad \mathrm{O}_{\text {tot }}=1.725
\end{aligned}
$$

Total Moles is sum of oxygen + nitrates + feed components:

$$
\begin{aligned}
\mathrm{Mol}_{\text {tot }}:= & \mathrm{O}_{\text {tot }}+\mathrm{Al}+\left(1+\mathrm{X}_{\mathrm{NO} 3}\right) \cdot(\mathrm{Na}+\mathrm{K})+\mathrm{Ca} \ldots \\
& +\mathrm{PO} 4+\mathrm{F}+\mathrm{SO} 4+\mathrm{Cl}+\mathrm{Zr}+\mathrm{B}+\mathrm{Fe}+\mathrm{Cd}+\mathrm{Cr}+\mathrm{Pb}+\mathrm{U} \quad \quad \mathrm{Mol}_{\text {tot }}=6.404
\end{aligned}
$$

3. Calculate mole percent sodium plus potassium:

Mole \% Na+K is:

$$
\mathrm{Mol} \%{ }_{\mathrm{NaK}}:=\frac{(\mathrm{Na}+\mathrm{K})}{\mathrm{Mol}_{\text {tot }}} \cdot 100 \cdot \% \quad \quad \mathrm{Mol} \%{ }_{\mathrm{NaK}}=4.747 \cdot \%
$$

4. Calculate weight of calcined solids:

Total calcine weight is the sum of (MW $\times$ moles):

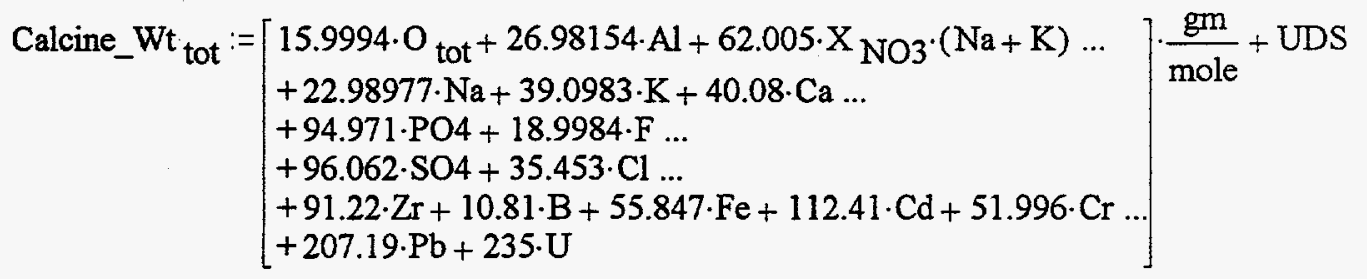

(Use average isotopic weight for uranium instead of 235, or weight conc $(g / L)$ of uranium instead of " $235 \mathrm{U}$ ", if available )

Calcine_Wt tot $=190.8 \cdot \mathrm{gm}$ (per liter of solution)

5. Estimated bulk density of calcined solids product and fines from plant or pilot plant data:

$$
\rho_{b \_p}=1.7 \cdot \frac{\mathrm{gm}}{\mathrm{cm}^{3}} \quad \text { (value given is for a typical Fluorinel/ } \mathrm{Na} \text { blend) }
$$

6. Conservatively estimate density of product and fines mixture based on expected product to fines ratio. Use $95 \%$ of product bulk density in most cases. Use $90 \%$ of product bulk density if P/F ratio is less than 2 .

$$
\begin{aligned}
& \rho_{\text {b_m }}:=.95 \cdot \rho_{\mathrm{b} \_\mathrm{p}} \\
& \rho_{\mathrm{b} \_\mathrm{m}}=1.615 \cdot \frac{\mathrm{gm}}{\mathrm{cm}^{3}}
\end{aligned}
$$

(P/F ratio for a FI/Na blend is about 3 to $4 \mathrm{~kg}$ product per $\mathrm{kg}$ fines so use $95 \%$ of product bulk density) 
7. Volume reduction factor $=$ ratio of volume of liquid feed to volume of calcine solid produced:

$$
\mathrm{VRF}:=\frac{\rho_{\mathrm{b} \_} \cdot \mathrm{m}^{\cdot 1000 \cdot \mathrm{cm}^{3} \cdot \text { liter }^{-1}}}{\text { Calcine_Wt } \text { tot } \text { liter }^{-1}}
$$

$$
\mathrm{VRF}=8.465 \frac{\text { liter_feed }}{\text { liter_calcine }}
$$

8. The VRF is usually corrected for feed additives (such as Calcium nitrate and aluminum nitrate) to give the ratio of volume of tank farm waste to volume of calcine solid produced. This allows a direct calculation of bin volumes from tank farm depletion.

\section{(Calculation basis $=1$ liter) $\quad V_{\text {totalfeed }}:=1 \cdot$ liter}

The calculated concentrations did not include volume due to additives, such as water in CaNO3_2, so:

$$
\begin{aligned}
& V_{\text {feed_additives }}:=0 \text {-liter for this case. } \\
& \mathrm{V}_{\text {tankfarm_waste }}:=\mathrm{V}_{\text {totalfeed }}-\mathrm{V}_{\text {feed_additives }} \\
& \mathrm{V}_{\text {tankfarm_waste }}=1 \cdot \text { liter } \\
& \mathrm{VRF}_{\mathrm{TF}}:=\mathrm{VRF} \cdot\left(\frac{\mathrm{V}_{\text {totalfeed }}-\mathrm{V}_{\text {feed_additives }}}{\mathrm{V}_{\text {totalfeed }}}\right) \\
& \mathrm{VRF}_{\mathrm{TF}}=8.465 \frac{\text { liter_tank_farm_waste }}{\text { liter_calcine }}
\end{aligned}
$$

9. Calculate heat generation rate of calcine from VRF and liquid heat generation rate:

$$
Q_{\text {liq }}:=5 \cdot \frac{\text { watt }}{\mathrm{m}^{3}} \quad \begin{aligned}
& \text { (see Appendix E for calculation of liquid heat generation) Use an } \\
& \text { estimated value since no radiochem was listed in Nby-08-90. }
\end{aligned}
$$

Calcine heat generation rate is then:

$\mathrm{Q}_{\text {calcine }}:=\mathrm{Q}_{\mathrm{liq}} \cdot \mathrm{VRF}$ if liquid heat generation was per volume of blend.

or

$Q_{\text {calcine }}:=Q_{\text {liq }} \cdot V R F T F$ if liquid heat generation was per volume of waste without cold additives.

Which for this case is:

$$
\begin{aligned}
& Q_{\text {calcine }}:=Q_{\text {liq }} \cdot V R F T F \\
& Q_{\text {calcine }}=42.327 \cdot \frac{\text { watt }}{\mathrm{m}^{3}}
\end{aligned}
$$




\section{EXAMPLE CALCULATION \#2}

\section{NWCF High Sodium Blended Feed of Evaporated WM-183/189 \\ (mixed with WM-188 heel) With Aluminum Nitrate}

Test Case Solution Content for 1 liter of Feed (without scrub recycle) Values from letters RJR-05-94 and RJR-07-94, references 19 and 20) ):

Solution is high sodium blend of Sodium Waste and Aluminum Nitrate:

$$
\begin{array}{llll}
\mathrm{F}:=.1575 \cdot \mathrm{mole} & \mathrm{Al}:=1.2139 \cdot \mathrm{mole} & \mathrm{Ca}:=.1099 \cdot \mathrm{mole} & \mathrm{Cr}:=.0101 \cdot \mathrm{mole} \\
\mathrm{SO} 4:=.006 \cdot \mathrm{mole} & \mathrm{Zr}:=.0172 \cdot \mathrm{mole} & \mathrm{B}:=.0999 \cdot \mathrm{mole} & \mathrm{Pb}:=\frac{.1911}{207.19} \cdot \mathrm{mole} \\
\mathrm{Cl}:=.0041 \cdot \mathrm{mole} & \mathrm{Na}:=.4708 \cdot \mathrm{mole} & \mathrm{Fe}:=.0363 \cdot \mathrm{mole} & \mathrm{U}:=\frac{.07897}{235} \cdot \mathrm{mole} \\
\mathrm{PO} 4:=\frac{.34505}{94.971} \cdot \mathrm{mole} & \mathrm{K}:=.0666 \cdot \mathrm{mole} & \mathrm{Cd}:=.0068 \cdot \mathrm{mole} \quad & \begin{array}{l}
\text { (Use ave isotopic weight for } \\
\text { uranium if analyses is available) }
\end{array}
\end{array}
$$

(Nitrate and acid concentrations in feed are not used in the calculation, but are given here for completeness)

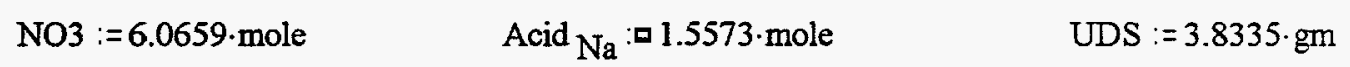

Calculated concentrations:

$$
\mathrm{PO} 4=0.004 \cdot \mathrm{mole} \quad \mathrm{Pb}=9.223 \cdot 10^{-4} \cdot \text { mole } \quad \mathrm{U}=3.36 \cdot 10^{-4} \cdot \mathrm{mole}
$$

Estimated calcine product bulk density from RJR-05-94: $\quad \rho_{\mathrm{b} \_\mathrm{p}}:=1.5 \cdot \frac{\mathrm{gm}}{\mathrm{cm}^{3}}$

Average Mole fraction of $\mathrm{Na}$ and $\mathrm{K}$ as nitrates based on analysis of calcine:

$$
\mathrm{X}_{\mathrm{NO} 3}:=0.66 \frac{\text { mole } \cdot \mathrm{NO}_{3}}{\text { mole }(\mathrm{Na}+\mathrm{K})} \quad \begin{aligned}
& (0.66 \pm 0.05 \text { based on analyses of pilot plant calcine without } \\
& \text { reducing agents, Reference } 7 . \text { Use pilot plant calcine data } \\
& \text { for } \left.\mathrm{NO}_{3} /(\mathrm{Na}+\mathrm{K}) \text { ratios if reducing agents such as sugar are used. }\right)
\end{aligned}
$$

\section{Calculations:}

1. Verify there is enough $\mathrm{Ca}, \mathrm{Na}$, and $\mathrm{K}$, to react with all $\mathrm{PO} 4, \mathrm{~F}, \mathrm{SO} 4$, and $\mathrm{Cl}$ so that none of the $\mathrm{PO} 4, \mathrm{~F}, \mathrm{SO} 4$, and $\mathrm{Cl}$ volatilize:

$$
\left[\left[2 \cdot \mathrm{Ca}+\left(1-\mathrm{X}_{\mathrm{NO} 3}\right) \cdot(\mathrm{Na}+\mathrm{K})\right] \geq(3 \cdot \mathrm{PO} 4+\mathrm{F}+2 \cdot \mathrm{SO} 4+\mathrm{Cl})\right]=1 \quad(=0 \text { for false, } 1 \text { for true })
$$

$\therefore$ True, therefore, there is sufficient $\mathrm{Ca}, \mathrm{Na}$, and $\mathrm{K}$ for all non-oxides assumed to form.

(This is somewhat conservative since it doesn't account for possible CdSO4) 
2. Calculate moles of oxygen and total moles:

There is enough $\mathrm{Ca}, \mathrm{Na}, \mathrm{K}$, and $\mathrm{Al}$, so the simplified equation for determining moles of oxygen is preferred to the detailed calculation of Appendix A:

Total moles of oxygen in oxides is therefore:

$$
\begin{aligned}
\mathrm{O}_{\text {tot }}:= & \frac{3}{2} \cdot \mathrm{Al}+\frac{1}{2} \cdot\left(1-\mathrm{X}_{\mathrm{NO} 3}\right) \cdot \mathrm{Na}+\frac{1}{2} \cdot\left(1-\mathrm{X}_{\mathrm{NO} 3}\right) \cdot \mathrm{K}+\mathrm{Ca}-\mathrm{SO} 4-\frac{1}{2} \cdot \mathrm{Cl}-\frac{3}{2} \cdot \mathrm{PO} 4-\frac{1}{2} \cdot \mathrm{F}+\mathrm{Cd} \ldots \\
& +2 \cdot \mathrm{Zr}+\frac{3}{2} \cdot \mathrm{B}+\frac{3}{2} \cdot \mathrm{Fe}+\frac{3}{2} \cdot \mathrm{Cr}+\mathrm{Pb}+3 \cdot \mathrm{U} \quad \mathrm{O}_{\text {tot }}=2.192
\end{aligned}
$$

Total Moles is sum of oxygen + nitrates + feed components:

$$
\begin{aligned}
\mathrm{Mol}_{\text {tot }}:= & \mathrm{O}_{\text {tot }}+\mathrm{Al}+\left(1+\mathrm{X}_{\mathrm{NO} 3}\right) \cdot(\mathrm{Na}+\mathrm{K})+\mathrm{Ca} \ldots \\
& +\mathrm{PO} 4+\mathrm{F}+\mathrm{SO} 4+\mathrm{Cl}+\mathrm{Zr}+\mathrm{B}+\mathrm{Fe}+\mathrm{Cd}+\mathrm{Cr}+\mathrm{Pb}+\mathrm{U} \quad \quad \mathrm{Mol}_{\text {tot }}=4.751
\end{aligned}
$$

3. Calculate mole percent sodium plus potassium:

Mole \% Na+K is:

$$
\mathrm{Mol} \% \mathrm{NaK}:=\frac{(\mathrm{Na}+\mathrm{K})}{\mathrm{Mol}_{\text {tot }}} \cdot 100 \% \quad \quad \mathrm{Mol} \% \mathrm{NaK}=11.31 \cdot \%
$$

4. Calculate weight of calcined solids:

Total calcine weight is the sum of (MW $x$ moles):

$$
\text { Calcine_Wt tot }:=\left[\begin{array}{ll}
15.9994 \cdot \mathrm{O}_{\text {tot }}+26.98154 \cdot \mathrm{Al}+62.005 \cdot \mathrm{X}_{\mathrm{NO} 3} \cdot(\mathrm{Na}+\mathrm{K}) \ldots \\
& +22.98977 \cdot \mathrm{Na}+39.0983 \cdot \mathrm{K}+40.08 \cdot \mathrm{Ca} \ldots \\
& +94.971 \cdot \mathrm{PO} 4+18.9984 \cdot \mathrm{F} \ldots \\
& +96.062 \cdot \mathrm{SO} 4+35.453 \cdot \mathrm{Cl} \ldots \\
& +91.22 \cdot \mathrm{Zr}+10.81 \cdot \mathrm{B}+55.847 \cdot \mathrm{Fe}+112.41 \cdot \mathrm{Cd}+51.996 \cdot \mathrm{Cr} \ldots \\
& +207.19 \cdot \mathrm{Pb}+235 \cdot \mathrm{U}
\end{array}\right] \cdot \frac{\mathrm{gm}}{\mathrm{mole}}+\mathrm{UDS}
$$

(Use average isotopic weight for uranium instead of 235 , or weight conc $(\mathrm{g} / \mathrm{L})$ of uranium instead of " $235 \mathrm{U}$ ", if available )

Calcine_Wt $\mathrm{tot}_{\mathrm{t}}=121.8 \cdot \mathrm{gm}$ (per liter of solution)

5. Estimated bulk density of calcined solids product and fines from plant or pilot plant data:

$$
P_{\mathrm{b} \_\mathrm{p}}=1.5 \cdot \frac{\mathrm{gm}}{\mathrm{cm}^{3}}
$$

6. Conservatively estimate density of product and fines mixture based on expected product to fines ratio. Use $95 \%$ of product bulk density in most cases. Use $90 \%$ of product bulk density if $P / F$ ratio is less than 2 .

$$
\begin{aligned}
& \rho_{\mathrm{b} \_\mathrm{m}}:=.90 \cdot \rho_{\mathrm{b} \_\mathrm{p}} \\
& \rho_{\mathrm{b} \_\mathrm{m}}=1.35 \cdot \frac{\mathrm{gm}}{\mathrm{cm}^{3}}
\end{aligned}
$$

(P/F ratio for an ANN/Na Blend is about $1 \mathrm{~kg}$ product per $\mathrm{kg}$ fines so use $90 \%$ of product bulk density) 
7. Volume reduction factor $=$ ratio of volume of liquid feed to volume of calcine solid produced:

$$
\text { VRF }:=\frac{\rho_{\mathrm{b}} \mathrm{m} \cdot 1000 \cdot \mathrm{cm}^{3} \cdot \text { liter }^{-1}}{\text { Calcine_Wt } \text { tot } \cdot \text { liter }^{-1}}
$$$$
\mathrm{VRF}=11.085 \frac{\text { liter_feed }}{\text { liter_calcine }} \text { " }
$$

8. The VRF is usually corrected for feed additives (such as Calcium nitrate and aluminum nitrate) to give the ratio of volume of tank farm waste to volume of calcine solid produced. This allows a direct calculation of bin volumes from tank farm depletion.

(Calculation basis was1 liter) $\mathrm{V}_{\text {totalfeed }}:=1 \cdot$ liter

The volumes of ANN, liquid $\mathrm{Ca}(\mathrm{NO} 3) 2$, and boric acid from RJR-05-94 (given as liters per liter of waste) are:

$$
\mathrm{V}_{\text {feed_additives }}:=\frac{.611+.018+.197}{(1.0+.611+.018+.197)} \cdot \text { liter per liter of total feed }
$$

Volume of tank farm waste in the blend is then:

$$
\begin{aligned}
& V_{\text {tankfarm_waste }}:=V_{\text {totalfeed }}-V_{\text {feed_additives }} \\
& V_{\text {tankfarm_waste }}=0.548 \cdot \text { liter } \\
& V_{T F}:=V R F \cdot\left(\frac{V_{\text {totalfeed }}-V_{\text {feed_additives }}}{V_{\text {totalfeed }}}\right) \\
& V_{T F} F_{T F}=6.071 \quad \frac{\text { liter_tank_farm_waste }}{\text { liter_calcine }} u \text { for the high sodium 188/183/189/ANN blended feed }
\end{aligned}
$$

9. Calculate heat generation rate of calcine from VRF and liquid heat generation rate:

$$
\begin{aligned}
& Q_{\text {liq }}:=2.350 \cdot \frac{\text { watt }}{\mathrm{m}^{3}} \quad \begin{array}{l}
\text { for the 188/183/189/ANN high sodium blend (see Appendix } E \text { ), } \\
\text { concentrations are per liquid volume of the blended feed, not } \\
\text { volume of tank farm waste. }
\end{array} \\
& Q_{\text {calcine }}:=Q_{\text {liq }} \cdot V R F \\
& Q_{\text {calcine }}=26.05 \cdot \frac{\text { watt }}{\mathrm{m}^{3}}
\end{aligned}
$$




\section{EXAMPLE CALCULATION \#3}

\section{0-cm Calciner Pilot Plant Run SBW4-46: Simulated High}

Sodium Blend of Aluminum Nitrate and Sodium Waste

Test Case Solution Content for 1 liter of Feed (without scrub recycle) (Values from test plan letter TJM-03-93, Reference 22). This run simulated a high sodium ANN/Na blend of ANN and WM-180:

$$
\begin{array}{llll}
\mathrm{F}:=0.013 \cdot \mathrm{mole} & \mathrm{Al}:=1.686 \cdot \mathrm{mole} & \mathrm{Ca}:=0.036 \cdot \mathrm{mole} & \mathrm{Cr}:=0.0 \cdot \mathrm{mole} \\
\mathrm{SO} 4:=0.017 \cdot \mathrm{mole} & \mathrm{Zr}:=0.0 \cdot \mathrm{mole} & \mathrm{B}:=0.15 \cdot \mathrm{mole} & \mathrm{Pb}:=0.0 \cdot \mathrm{mole} \\
\mathrm{Cl}:=0.010 \cdot \mathrm{mole} & \mathrm{Na}:=0.623 \cdot \mathrm{mole} & \mathrm{Fe}:=0.005 \cdot \mathrm{mole} & \mathrm{U}:=0.0 \cdot \mathrm{mole}
\end{array}
$$$$
\text { PO4 }:=0.0 \cdot \mathrm{mole} \quad \mathrm{K}:=0.056 \mathrm{~mole} \quad \mathrm{Cd}:=0.0 \cdot \mathrm{mole} \quad \text { (Use ave isotopic weight for }
$$$$
\text { uranium if analyses is available) }
$$

(Nitrate and acid concentrations in feed are not used in the calculation, but are given here for completeness)

$$
\mathrm{NO} 3:=5.65 \cdot \mathrm{mole}_{\mathrm{Nad}} \mathrm{Na}:=0.620 \cdot \mathrm{mole} \quad \mathrm{UDS}:=0.0 \cdot \mathrm{gm}
$$

Calcine product bulk density from pilot plant run SBW4-46: $\quad \rho_{b} \_:=1.75 \cdot \frac{\mathrm{gm}}{\mathrm{cm}^{3}}$

Average Mole fraction of $\mathrm{Na}$ and $\mathrm{K}$ as nitrates based on analysis of calcine:

$$
\mathrm{X}_{\mathrm{NO} 3}:=0.66 \frac{\text { mole } \cdot \mathrm{NO}_{3}}{\text { mole } \cdot(\mathrm{Na}+\mathrm{K})} \quad \begin{aligned}
& \begin{array}{l}
(0.66 \pm 0.05 \text { based on analyses of pilot plant calcine without } \\
\text { reducing agents, Reference } 7 . \text { Use pilot plant calcine data } \\
\text { for } \left.\mathrm{NO}_{3} /(\mathrm{Na}+\mathrm{K}) \text { ratios if reducing agents such as sugar are used. }\right)
\end{array}
\end{aligned}
$$

\section{Calculation:}

1. Verify there is enough $\mathrm{Ca}, \mathrm{Na}$, and $\mathrm{K}$, to react with all $\mathrm{PO}$, $\mathrm{F}, \mathrm{SO}$, and $\mathrm{Cl}$ so that none of the $\mathrm{PO} 4, \mathrm{~F}$, SO4, and $\mathrm{Cl}$ volatilize:

$$
\left[\left[2 \cdot \mathrm{Ca}+\left(1-\mathrm{X}_{\mathrm{NO} 3}\right) \cdot(\mathrm{Na}+\mathrm{K})\right] \geq(3 \cdot \mathrm{PO} 4+\mathrm{F}+2 \cdot \mathrm{SO} 4+\mathrm{Cl})\right]=1 \quad(=0 \text { for false, } 1 \text { for true })
$$

$\therefore$ True, therefore, there is sufficient $\mathrm{Ca}, \mathrm{Na}$, and $\mathrm{K}$ for all non-oxides assumed to form. (This is somewhat conservative since it doesn't account for possible CdSO4) 
2. Calculate moles of oxygen and total moles:

There is enough $\mathrm{Ca}, \mathrm{Na}, \mathrm{K}$, and $\mathrm{Al}$, so the simplified equation for determining moles of oxygen is preferred to the detailed calculation of Appendix A:

Total moles of oxygen in oxides is therefore:

$$
\begin{aligned}
\mathrm{O}_{\text {tot }}:= & \frac{3}{2} \cdot \mathrm{Al}+\frac{1}{2} \cdot\left(1-\mathrm{X}_{\mathrm{NO} 3}\right) \cdot \mathrm{Na}+\frac{1}{2} \cdot\left(1-\mathrm{X}_{\mathrm{NO} 3}\right) \cdot \mathrm{K}+\mathrm{Ca}-\mathrm{SO} 4-\frac{1}{2} \cdot \mathrm{Cl}-\frac{3}{2} \cdot \mathrm{PO} 4-\frac{1}{2} \cdot \mathrm{F}+\mathrm{Cd} \ldots \\
& +2 \cdot \mathrm{Zr}+\frac{3}{2} \cdot \mathrm{B}+\frac{3}{2} \cdot \mathrm{Fe}+\frac{3}{2} \cdot \mathrm{Cr}+\mathrm{Pb}+3 \cdot \mathrm{U} \\
& \mathrm{O}_{\text {tot }}=2.884
\end{aligned}
$$

Total Moles is sum of oxygen + nitrates + feed components:

$$
\begin{aligned}
& \mathrm{Mol}_{\text {tot }}:=\mathrm{O}_{\text {tot }}+\mathrm{Al}+\left(1+\mathrm{X}_{\mathrm{NO} 3}\right) \cdot(\mathrm{Na}+\mathrm{K})+\mathrm{Ca} \ldots \\
& +\mathrm{PO} 4+\mathrm{F}+\mathrm{SO} 4+\mathrm{Cl}+\mathrm{Zr}+\mathrm{B}+\mathrm{Fe}+\mathrm{Cd}+\mathrm{Cr}+\mathrm{Pb}+\mathrm{U} \\
& \mathrm{Mol}_{\text {tot }}=5.929
\end{aligned}
$$

3. Calculate mole percent sodium plus potassium

Mole \% Na+K is:

$$
\mathrm{Mol}{ }_{\mathrm{NaK}}:=\frac{(\mathrm{Na}+\mathrm{K})}{\mathrm{Mol}_{\text {tot }}} \cdot 100 \% \quad \quad \mathrm{Mol} \% \mathrm{NaK}=11.45 \cdot \%
$$

4. Calculate weight of calcined solids:

Total calcine weight is the sum of (MW $\times$ moles):

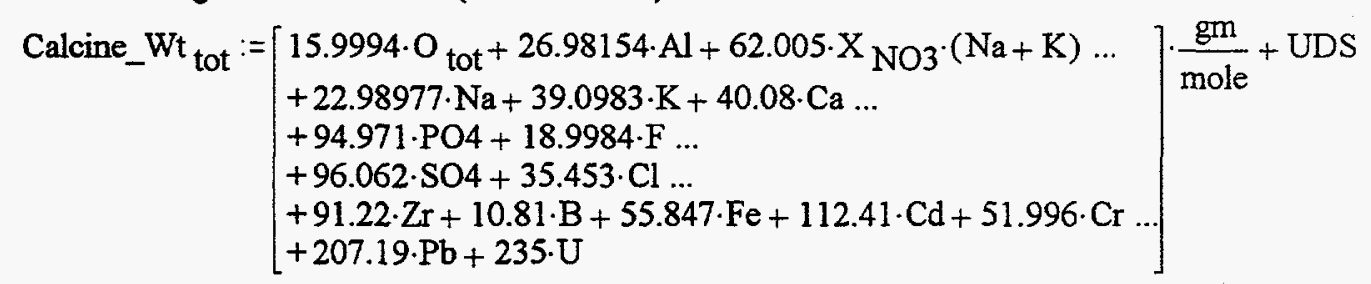

(Use average isotopic weight for uranium instead of 235 , or weight conc ( $g / L)$ of uranium instead of " $235 \mathrm{U}$ ", if available )

Calcine_Wt $\mathrm{tot}=141.5 \cdot \mathrm{gm}$ (per liter of solution)

5. Estimated bulk density of calcined solids product and fines from plant or pilot plant data:

$$
\rho_{\mathrm{b} \_\mathrm{p}}=1.75 \cdot \frac{\mathrm{gm}}{\mathrm{cm}^{3}} \quad \text { (average for pilot plant Run SBW4-46) }
$$

6. Conservatively estimate density of product and fines mixture based on expected product to fines ratio. Use $95 \%$ of product bulk density in most cases. Use $90 \%$ of product bulk density if P/F ratio is less than 2.

$$
\begin{array}{ll}
\rho_{\mathrm{b}_{-} \mathrm{m}}:=.90 \cdot \rho_{\mathrm{b} \_} & \text {(P/F ratio for the run was } 1.45, \text { so use } 90 \% \text { of } \\
\text { product bulk density for product and fines mixture) }
\end{array}
$$


7. Volume reduction factor $=$ ratio of volume of liquid feed to volume of calcine solid produced:

$$
\mathrm{VRF}:=\frac{\rho_{\mathrm{b} \_\mathrm{m}} \cdot 1000 \cdot \mathrm{cm}^{3} \cdot \operatorname{liter}^{-1}}{\text { Calcine_Wt }_{\text {tot }} \text { liter }^{-1}} \quad \mathrm{VRF}=11.129 \frac{\text { liter_feed }}{\text { liter_calcine }}
$$

8. The VRF is usually corrected for feed additives (such as Calcium nitrate and aluminum nitrate) to give the ratio of volume of tank farm waste to volume of calcine solid produced. This allows a direct calculation of bin volumes from tank farm depletion.

(Calculation basis was 1 liter) $\mathrm{V}$ totalfeed $:=1$ liter

The feed concentrations for SBW4-46 in TJM-03-93 were based on blending 2.26 vol ANN to 1 Vol 180 , and assumed volumes of liquid $\mathrm{Ca}(\mathrm{NO} 3) 2$ and boric acid were negligible:

$$
\begin{array}{ll}
\mathrm{V}_{\text {feed_additives }}:=\frac{2.26+0+0}{(1.0+2.26+0+0)} \cdot \text { liter } & \text { per liter of total feed } \\
V_{\text {feed_additives }}=0.693 \cdot \text { liter } & \text { per liter of total feed }
\end{array}
$$

The volume of tank farm waste in the blend is then:

$$
\begin{aligned}
& V_{\text {tankfarm_waste }}:=V_{\text {totalfeed }}-V_{\text {feed_additives }} \\
& V_{\text {tankfarm_waste }}=0.307 \cdot \text { liter } \\
& V_{T F} \text { TF }:=V R F \cdot\left(\frac{V_{\text {totalfeed }}-V_{\text {feed_additives }}}{V_{\text {totalfeed }}}\right) \\
& V_{\text {RF }} \text { TF }=3.414 \quad \frac{\text { volumes_tank_farm_waste }}{\text { volume_calcine }}
\end{aligned}
$$

9. Calculate heat generation rate of calcine from VRF and liquid heat generation rate:

$$
\begin{aligned}
& Q_{\text {liq }}:=0.322 \cdot \frac{\text { watt }}{\mathrm{m}^{3}} \quad \begin{array}{l}
\text { for } W M-180 \text { tank farm waste (see Appendix } E \text { ), so use VRF } \\
\text { rather than VRF. }
\end{array} \\
& Q_{\text {calcine }}:=Q_{\text {liq }} \cdot V R F \text { TF } \\
& Q_{\text {calcine }}=1.099 \cdot \frac{\text { watt }}{\mathrm{m}^{3}}
\end{aligned}
$$


Appendix D

Comparision of Updated Calculation Method with Previous Methods

D-1 


\section{Appendix D \\ Comparison the Updated of Sodium Plus Potassium Content and Weight of Calcined Solids with Previous Methods}

Wanted: Compare updated calculation methods with the previous methods and develop equations for converting old method numbers to new numbers.

Method: 1. a. The previous methods did not apply if species volatilized, so the simplified oxygen calculation can be used for comparison.

b. Calculate mole \% $\mathrm{Na}+\mathrm{K}$ by updated method and the method in letter Nby-08-90.

c. Derive an equation relating the two mole percents.

2. Calcule and compare weight of calcine solids by the updated method and the method in ENICO-1100.

Given:

Test Case Solution Content for 1 liter of Solution-Case Nby1 (Values from letter Nby-08-90, case 1):

Solution is a $5: 1$ blend of Typical Fluorinel and $\mathrm{Na}$ waste.

$$
\begin{array}{llll}
\mathrm{F}:=\frac{5 \cdot 2.27+0.049}{6} \cdot \text { mole } & \mathrm{Al}:=\frac{5 \cdot 0.36+.45}{6} \cdot \text { mole } & \mathrm{Ca}:=0.7 \cdot \mathrm{F} & \mathrm{Cr}:=.0 \cdot \mathrm{mole} \\
\mathrm{SO} 4:=\frac{5 \cdot 0.079+.048}{6} \cdot \mathrm{mole} & \mathrm{Zr}:=\frac{5 \cdot 0.29+0}{6} \cdot \mathrm{mole} & \mathrm{B}:=\frac{5 \cdot 0.17+0.024}{6} \cdot \mathrm{mole} & \mathrm{Pb}:=0 \cdot \mathrm{mole} \\
\mathrm{Cl}:=\frac{5 \cdot 0.0016+0.031}{6} \cdot \mathrm{mole} & \mathrm{Na}:=\frac{5 \cdot 0.028+1.46}{6} \cdot \mathrm{mole} \mathrm{Fe}:=\frac{5 \cdot 0.0072+0.023}{6} \cdot \mathrm{mole} \quad \mathrm{U}:=0 \cdot \mathrm{mole} \\
\mathrm{PO} 4:=.0 \cdot \mathrm{mole} & \mathrm{K}:=\frac{5 \cdot 0.0028+.21}{6} \cdot \mathrm{mole} \mathrm{Cd}:=\frac{5 \cdot 0.11+0}{6} \cdot \mathrm{mole} \\
\mathrm{NO} 3:=\left(\frac{5 \cdot 2.37+4.47}{6}\right) \cdot \mathrm{mole}^{2}+2 \cdot \mathrm{Ca} \quad \text { Acid } \mathrm{Na}:=\frac{5 \cdot 2.04+1.69}{6} \cdot \mathrm{mole} \quad \mathrm{UDS}:=\frac{5 \cdot 2+1}{6} \cdot \mathrm{gm}
\end{array}
$$

Calculated concentrations for 5:1 blend:

$$
\begin{array}{llll}
\mathrm{F}=1.9 \cdot \mathrm{mole} & \mathrm{Al}=0.375 \cdot \mathrm{mole} & \mathrm{Ca}=1.33 \cdot \mathrm{mole} & \mathrm{Cr}=0 \cdot \mathrm{mole} \\
\mathrm{SO} 4=0.074 \cdot \mathrm{mole} & \mathrm{Zr}=0.242 \cdot \mathrm{mole} & \mathrm{B}=0.146 \cdot \mathrm{mole} & \mathrm{Pb}=0 \cdot \text { mole } \\
\mathrm{Cl}=0.006 \cdot \mathrm{mole} & \mathrm{Na}=0.267 \cdot \mathrm{mole} & \mathrm{Fe}=0.01 & \mathrm{U}=0 \cdot \mathrm{mole} \\
\mathrm{PO} 4=0 \cdot \mathrm{mole} & \mathrm{K}=0.037 \cdot \mathrm{mole} & \mathrm{Cd}=0.092 \cdot \mathrm{mole} & \mathrm{UDS}=1.833 \cdot \mathrm{gm}
\end{array}
$$

(Nitrate and acid concentrations in feed are not used in the calculation, but are given here for completeness)

$\mathrm{NO} 3=5.38 \cdot \mathrm{mole} \quad \mathrm{Na}+\mathrm{K}=0.304 \cdot \mathrm{mole} \quad$ Acid $_{\mathrm{Na}}=1.982 \cdot$ mole 


\section{Calculate $\mathrm{Na}+\mathrm{K}$ mole percent using updated method and Newby's method.}

\section{A. Updated Method:}

Check to make sure there is enough $\mathrm{Ca}, \mathrm{Na}, \mathrm{K}$, and $\mathrm{Al}$ to form compounds with all $\mathrm{PO} 4, \mathrm{~F}$, $\mathrm{SO}$, and $\mathrm{Cl}$ :

$$
\left.\left[\left[2 \cdot \mathrm{Ca}+\left(1-\mathrm{X}_{\mathrm{NO} 3}\right) \cdot(\mathrm{Na}+\mathrm{K})\right] \geq(3 \cdot \mathrm{PO} 4+\mathrm{F}+2 \cdot \mathrm{SO} 4+\mathrm{Cl})\right]=1 \quad \text { (=0 for false, } 1 \text { for true }\right)
$$

(This is somewhat conservative since it doesn't account for possible CdSO4)

Average Mole fraction of $\mathrm{Na}$ and $\mathrm{K}$ as nitrates based on analysis of calcine:

$$
\mathrm{X}_{\mathrm{NO} 3}=0.66 \frac{\mathrm{mole} \cdot \mathrm{NO}_{3}}{\mathrm{~mole} \cdot(\mathrm{Na}+\mathrm{K})}
$$

Total Moles of oxygen for updated method:

$$
\begin{aligned}
\mathrm{O}_{\text {tot }}:= & \frac{3}{2} \cdot \mathrm{Al}+\frac{1}{2} \cdot\left(1-\mathrm{X}_{\mathrm{NO} 3}\right) \cdot \mathrm{Na}+\frac{1}{2} \cdot\left(1-\mathrm{X}_{\mathrm{NO} 3}\right) \cdot \mathrm{K}+\mathrm{Ca}-\mathrm{SO} 4-\frac{1}{2} \cdot \mathrm{Cl}-\frac{3}{2} \cdot \mathrm{PO} 4-\frac{1}{2} \cdot \mathrm{F}+\mathrm{Cd} \ldots \\
& +2 \cdot \mathrm{Zr}+\frac{3}{2} \cdot \mathrm{B}+\frac{3}{2} \cdot \mathrm{Fe}+\frac{3}{2} \cdot \mathrm{Cr}+\mathrm{Pb}+3 \cdot \mathrm{U} \quad \mathrm{O}_{\text {tot }}=1.725 \cdot \mathrm{mole}
\end{aligned}
$$

Total Moles is sum of oxygen + nitrates + feed components- volatiles:

$$
\begin{array}{rlrl}
\mathrm{Mol}_{\text {tot }}:=\mathrm{O}_{\text {tot }}+\mathrm{Al}+\left(1+\mathrm{X}_{\mathrm{NO} 3}\right) \cdot(\mathrm{Na}+\mathrm{K})+\mathrm{Ca} \ldots & \\
& +\mathrm{PO} 4+\mathrm{F}+\mathrm{SO} 4+\mathrm{Cl}+\mathrm{Zr}+\mathrm{B}+\mathrm{Fe}+\mathrm{Cd}+\mathrm{Cr}+\mathrm{Pb}+\mathrm{U} & & \mathrm{Mol}_{\text {tot }}=6.404 \cdot \mathrm{mole} \\
\mathrm{Mol} \% \mathrm{NaK}:=\frac{(\mathrm{Na}+\mathrm{K})}{\mathrm{Mol} \text { tot }} \cdot 100 \% & & \mathrm{Mol}^{2} \mathrm{NaK}=4.747 \cdot \%
\end{array}
$$

\section{B. Calculate mole percent using Newby's method:}

B.J.Newby Method for $\mathrm{Na}+\mathrm{K}$ mole \%(letter Nby-08-90), which assumes no nitrates and neglects species less than about 0.05 molar:

$$
\begin{array}{ll}
\mathrm{O}_{\text {nby }}:=\frac{1}{2} \cdot(\mathrm{Na}+\mathrm{K})+\frac{3}{2} \cdot \mathrm{Al}+\mathrm{Ca}-\frac{1}{2} \cdot \mathrm{F}-\mathrm{SO} 4+2 \cdot \mathrm{Zr}+\mathrm{Cd}+\frac{3}{2} \cdot \mathrm{B} & \mathrm{O}_{\mathrm{nby}}=1.814 \\
\mathrm{Mol}_{\text {nby }}:=\mathrm{O}_{\mathrm{nby}}+\mathrm{Al}+\mathrm{Na}+\mathrm{K}+\mathrm{Ca}+\mathrm{F}+\mathrm{SO} 4+\mathrm{Zr}+\mathrm{B}+\mathrm{Cd} & \mathrm{Mol}_{\mathrm{nby}}=6.276 \\
{ }_{\mathrm{Mol} \%}{ }_{\mathrm{NaK} \_n b y}:=\frac{(\mathrm{Na}+\mathrm{K})}{\mathrm{Mol}_{\text {nby }}} \cdot 100 \% & \mathrm{Mol} \%{ }^{\mathrm{NaK}} \mathrm{nby}=4.844 \cdot \%
\end{array}
$$

The relative difference between methods is: $\frac{\mathrm{Mol} \% \mathrm{NaK}_{-} \text {nby }-\mathrm{Mol} \% \mathrm{NaK}}{\mathrm{Mol} \% \mathrm{NaK}} \cdot 100 \cdot \%=2.042 \cdot \%$

The updated method gives a smaller mole percent than Newby's method for the same feed solution. This is because Newby's method has fewer total moles due to 1) no nitrates were assumed in the calcine, and 2) only major feed components were used and others were neglected. 


\section{Relate mole percent value of updated method to that of Newby's method:}

Since the mole percent sodium plus potassium values calculated using Newby's method are available for most previous flowsheets, it is desirable to have a quick way to convert between them and the updated method without having to use the entire feed composition. The following is the derivation of an equation which gives an approximate value of mole percent for the updated method which requires knowing only the Newby mole percent.

Total Moles of oxygen for updated method:

$$
\begin{aligned}
\mathrm{O}_{\text {tot }}:= & \frac{3}{2} \cdot \mathrm{Al}+\frac{1}{2} \cdot\left(1-\mathrm{X}_{\mathrm{NO} 3}\right) \cdot \mathrm{Na}+\frac{1}{2} \cdot\left(1-\mathrm{X}_{\mathrm{NO} 3}\right) \cdot \mathrm{K}+\mathrm{Ca}-\mathrm{SO} 4-\frac{1}{2} \cdot \mathrm{Cl}-\frac{3}{2} \cdot \mathrm{PO} 4-\frac{1}{2} \cdot \mathrm{F}+\mathrm{Cd} \ldots \\
& +2 \cdot \mathrm{Zr}+\frac{3}{2} \cdot \mathrm{B}+\frac{3}{2} \cdot \mathrm{Fe}+\frac{3}{2} \cdot \mathrm{Cr}+\mathrm{Pb}+3 \cdot \mathrm{U}
\end{aligned}
$$

Total Moles is sum of oxygen + nitrates + feed components:

$$
\begin{aligned}
\mathrm{Mol}_{\text {tot }}:= & \mathrm{O}_{\text {tot }}+\mathrm{Al}+\left(1+\mathrm{X}_{\mathrm{NO} 3}\right) \cdot(\mathrm{Na}+\mathrm{K})+\mathrm{Ca} \ldots \\
& +\mathrm{PO} 4+\mathrm{F}+\mathrm{SO} 4+\mathrm{Cl}+\mathrm{Zr}+\mathrm{B}+\mathrm{Fe}+\mathrm{Cd}+\mathrm{Cr}+\mathrm{Pb}+\mathrm{U}
\end{aligned}
$$

by substitution for total moles of oxygen, yields

$$
\begin{aligned}
\mathrm{Mol}_{\text {tot }}:= & \frac{3}{2} \cdot \mathrm{Al}+\frac{1}{2} \cdot\left(1-\mathrm{X}_{\mathrm{NO} 3}\right) \cdot \mathrm{Na}+\frac{1}{2} \cdot\left(1-\mathrm{X}_{\mathrm{NO} 3}\right) \cdot \mathrm{K}+\mathrm{Ca}-\mathrm{SO} 4-\frac{1}{2} \cdot \mathrm{Cl}-\frac{3}{2} \cdot \mathrm{PO} 4-\frac{1}{2} \cdot \mathrm{F}+\mathrm{Cd} \ldots \\
& +2 \cdot \mathrm{Zr}+\frac{3}{2} \cdot \mathrm{B}+\frac{3}{2} \cdot \mathrm{Fe}+\frac{3}{2} \cdot \mathrm{Cr}+\mathrm{Pb}+3 \cdot \mathrm{U}+\left[\mathrm{Al}+\left(1+\mathrm{X}_{\mathrm{NO} 3}\right) \cdot(\mathrm{Na}+\mathrm{K})+\mathrm{Ca}\right] \ldots \\
& +\mathrm{PO} 4+\mathrm{F}+\mathrm{SO} 4+\mathrm{Cl}+\mathrm{Zr}+\mathrm{B}+\mathrm{Fe}+\mathrm{Cd}+\mathrm{Cr}+\mathrm{Pb}+\mathrm{U}
\end{aligned}
$$

simplifies to

$$
\begin{aligned}
\mathrm{Mol}_{\text {tot }}:= & 3 \cdot \mathrm{Zr}+\frac{5}{2} \cdot \mathrm{B}+\frac{5}{2} \cdot \mathrm{Fe}+2 \cdot \mathrm{Cd}+\frac{3}{2} \cdot \mathrm{K}+\frac{1}{2} \cdot \mathrm{K} \cdot \mathrm{X}_{\mathrm{NO} 3}+\frac{3}{2} \cdot \mathrm{Na}+\frac{1}{2} \cdot \mathrm{X}_{\mathrm{NO} 3} \cdot \mathrm{Na} \ldots \\
& +\frac{1}{2} \cdot \mathrm{F}+4 \cdot \mathrm{U}+\frac{5}{2} \cdot \mathrm{Cr}+2 \cdot \mathrm{Pb}+2 \cdot \mathrm{Ca}+\frac{1}{2} \cdot \mathrm{Cl}-\frac{1}{2} \cdot \mathrm{PO} 4+\frac{5}{2} \cdot \mathrm{Al}
\end{aligned}
$$




\section{Relate mole percent value of updated method to that of Newby's method (cont.):}

B.J.Newby Method for $\mathrm{Na}+\mathrm{K}$ mole \%(letter Nby-08-90), which assumes no nitrates, and neglects species less than about 0.05 molar:

$$
\begin{aligned}
& \mathrm{O}_{\text {nby }}:=\frac{1}{2} \cdot(\mathrm{Na}+\mathrm{K})+\frac{3}{2} \cdot \mathrm{Al}+\mathrm{Ca}-\frac{1}{2} \cdot \mathrm{F}-\mathrm{SO} 4+2 \cdot \mathrm{Zr}+\mathrm{Cd}+\frac{3}{2} \cdot \mathrm{B} \\
& \mathrm{Mol}_{\text {nby }}:=\mathrm{O}_{\text {nby }}+\mathrm{Al}+\mathrm{Na}+\mathrm{K}+\mathrm{Ca}+\mathrm{F}+\mathrm{SO} 4+\mathrm{Zr}+\mathrm{B}+\mathrm{Cd}
\end{aligned}
$$

Substituting for moles of oxygen:

$$
\begin{aligned}
\mathrm{Mol}_{\text {nby }}:= & {\left[\frac{1}{2} \cdot(\mathrm{Na}+\mathrm{K})+\frac{3}{2} \cdot \mathrm{Al}+\mathrm{Ca}-\frac{1}{2} \cdot \mathrm{F}-\mathrm{SO} 4+2 \cdot \mathrm{Zr}+\mathrm{Cd}+\frac{3}{2} \cdot \mathrm{B}\right] \ldots } \\
& +\mathrm{Al}+\mathrm{Na}+\mathrm{K}+\mathrm{Ca}+\mathrm{F}+\mathrm{SO} 4+\mathrm{Zr}+\mathrm{B}+\mathrm{Cd}
\end{aligned}
$$

simplifies to

$$
\mathrm{Mol}_{\text {nby }}:=\frac{3}{2} \cdot \mathrm{Na}+\frac{3}{2} \cdot \mathrm{K}+\frac{5}{2} \cdot \mathrm{Al}+2 \cdot \mathrm{Ca}+\frac{1}{2} \cdot \mathrm{F}+3 \cdot \mathrm{Zr}+2 \cdot \mathrm{Cd}+\frac{5}{2} \cdot \mathrm{B}
$$

The difference in total moles for the new method versus Newby's method is:

$$
\mathrm{Mol}_{\text {dif }}:=\mathrm{Mol}_{\text {tot }}-\mathrm{Mol}_{\text {nby }}
$$

Substituting for the equations for total moles:

$$
\left.\begin{array}{rl}
\mathrm{Mol}_{\mathrm{dif}}:= & {\left[\begin{array}{l}
3 \cdot \mathrm{Zr}+\frac{5}{2} \cdot \mathrm{B}+\frac{5}{2} \cdot \mathrm{Fe}+2 \cdot \mathrm{Cd}+\frac{3}{2} \cdot \mathrm{K}+\frac{1}{2} \cdot \mathrm{K} \cdot \mathrm{X}_{\mathrm{NO} 3}+\frac{3}{2} \cdot \mathrm{Na}+\frac{1}{2} \cdot \mathrm{X}_{\mathrm{NO} 3} \cdot \mathrm{Na} \ldots \\
+\frac{1}{2} \cdot \mathrm{F}+4 \cdot \mathrm{U}+\frac{5}{2} \cdot \mathrm{Cr}+2 \cdot \mathrm{Pb}+2 \cdot \mathrm{Ca}+\frac{1}{2} \cdot \mathrm{Cl}-\frac{1}{2} \cdot \mathrm{PO} 4+\frac{5}{2} \cdot \mathrm{Al}
\end{array}\right] \ldots} \\
& +-1 \cdot\left(\frac{3}{2} \cdot \mathrm{Na}+\frac{3}{2} \cdot \mathrm{K}+\frac{5}{2} \cdot \mathrm{Al}+2 \cdot \mathrm{Ca}+\frac{1}{2} \cdot \mathrm{F}+3 \cdot \mathrm{Zr}+2 \cdot \mathrm{Cd}+\frac{5}{2} \cdot \mathrm{B}\right)
\end{array}\right]
$$

simplifies to

$$
\mathrm{Mol}_{\text {dif }}:=\frac{5}{2} \cdot \mathrm{Fe}+\frac{1}{2} \cdot \mathrm{X}_{\mathrm{NO} 3} \cdot(\mathrm{Na}+\mathrm{K})+4 \cdot \mathrm{U}+\frac{5}{2} \cdot \mathrm{Cr}+2 \cdot \mathrm{Pb}+\frac{1}{2} \cdot \mathrm{Cl}-\frac{1}{2} \cdot \mathrm{PO} 4
$$


Updated mole percent is:

$$
\mathrm{Mol} \% \mathrm{NaK}:=\frac{(\mathrm{Na}+\mathrm{K})}{\mathrm{Mol}_{\text {tot }}} \cdot 100 \cdot \% \text { ! }
$$

Substituting for total moles in terms of Newby's moles and the difference:

$$
\mathrm{Mol} \% \mathrm{NaK}:=\frac{(\mathrm{Na}+\mathrm{K})}{\mathrm{Mol}_{\text {nby }}+\mathrm{Mol}_{\text {dif }}} \cdot 100 \% \text { d }
$$

Substituting for terms for Newby's moles and the difference:

$$
{ }_{\mathrm{Mol}}{ }_{\mathrm{NaK}}:=\frac{(\mathrm{Na}+\mathrm{K})}{\left[\frac{(\mathrm{Na}+\mathrm{K})}{\mathrm{Mol} \% \mathrm{NaK} \_\mathrm{nby}} \cdot 100\right]+\left[\frac{5}{2} \cdot \mathrm{Fe}+\frac{1}{2} \cdot \mathrm{X}_{\mathrm{NO} 3} \cdot(\mathrm{Na}+\mathrm{K})+4 \cdot \mathrm{U}+\frac{5}{2} \cdot \mathrm{Cr}+2 \cdot \mathrm{Pb}+\frac{1}{2} \cdot \mathrm{Cl}-\frac{1}{2} \cdot \mathrm{PO} 4\right]} \cdot 1000
$$

rearranges to

$$
\mathrm{Mol}^{\mathrm{NaK}} \mathrm{NaK} \frac{\mathrm{Mol} \% \mathrm{NaK}_{\mathrm{nby}}}{100+\frac{\mathrm{Mol} \% \mathrm{NaK} \_\mathrm{nby}}{(\mathrm{Na}+\mathrm{K})} \cdot\left[\frac{1}{2} \cdot \mathrm{X}_{\mathrm{NO} 3} \cdot(\mathrm{Na}+\mathrm{K})+\frac{5}{2} \cdot \mathrm{Fe}+4 \cdot \mathrm{U}+\frac{5}{2} \cdot \mathrm{Cr}+2 \cdot \mathrm{Pb}+\frac{1}{2} \cdot \mathrm{Cl}-\frac{1}{2} \cdot \mathrm{PO} 4\right]} \cdot 100_{0}
$$

If $\mathrm{Fe}, \mathrm{U}, \mathrm{Cr}, \mathrm{Pb}, \mathrm{Cl}$ and $\mathrm{PO} 4$ are small (they are usually less than 0.02 molar), then the mole\% can be approximated from Newby's mole \% by:

$$
\text { Mol\% NaK_approx }:=\frac{\mathrm{Mol} \% \mathrm{NaK} \text { nby }}{100 \cdot \%+\frac{\mathrm{Mol} \% \mathrm{NaK} \text { nby }}{(\mathrm{Na}+\mathrm{K})} \cdot\left[\frac{1}{2} \cdot \mathrm{X}_{\mathrm{NO} 3} \cdot(\mathrm{Na}+\mathrm{K})\right]} \cdot 100 \%
$$

Which simplifies to:

$$
\text { Mol\% NaK_approx }:=\frac{\mathrm{Mol} \% \mathrm{NaK}_{\text {nby }}}{100 \%+\mathrm{Mol} \% \mathrm{NaK}_{\text {nby }} \cdot\left(\frac{1}{2} \cdot \mathrm{X}_{\mathrm{NO} 3}\right)} \cdot 100 \cdot \%
$$

For the test case the calculated values are:

$$
\begin{aligned}
& \mathrm{Mol} \% \mathrm{NaK}_{\text {nby }}=4.844 \cdot \% \\
& \mathrm{Mol} \% \mathrm{NaK}=4.747 \cdot \% \\
& \mathrm{Mol} \% \mathrm{NaK}_{\text {approx }}=4.768 \cdot \%
\end{aligned}
$$

Relative error of the approximate solution for the test case is:

$$
\text { Error approx }:=\frac{\text { Mol\% }{ }^{N a K} \text { approx }-\mathrm{Mol} \% \mathrm{NaK}}{\mathrm{Mol} \% \mathrm{NaK}} \cdot 100 . \% \quad \text { Error approx }=0.437 . \%
$$




\section{Compare updated equation for weight of calcine with to previous method.}

Updated equation for weight of calcine, assuming that nothing volatilizes is:

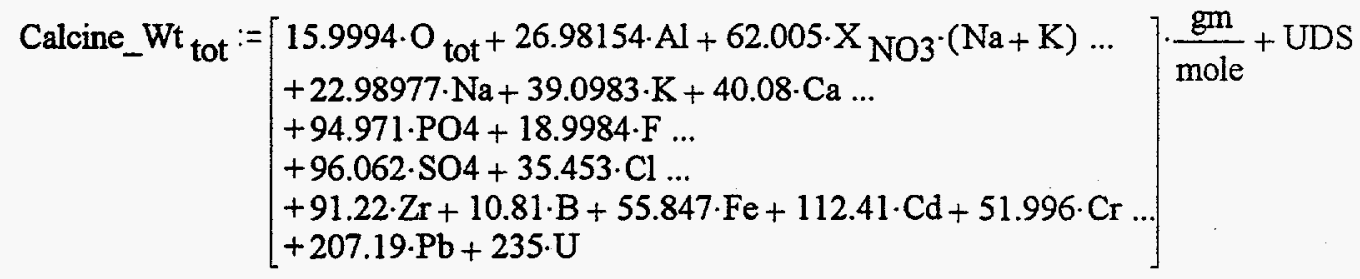

Calcine_Wt tot $=190.8 \cdot \mathrm{gm}$ (of calcine formed per liter of solution)

Check calculated weight percent of NO3 in calcine to see how it compares:

$$
\begin{aligned}
& \mathrm{X}_{\mathrm{NO} 3}=0.66 \\
& { }^{\mathrm{Wt}}{ }_{\mathrm{NO} 3}:=62.005 \cdot \frac{\mathrm{gm}}{\mathrm{mole}} \cdot \mathrm{X}_{\mathrm{NO}} \cdot(\mathrm{Na}+\mathrm{K}) \quad \mathrm{Wt}_{\mathrm{NO} 3}=12.441 \cdot \mathrm{gm} \text { (of NO3 formed per liter of solution) } \\
& \mathrm{Wt}^{2} \mathrm{NO} \text { : }:=\frac{\mathrm{Wt} \text { NO3 }}{\text { Calcine_Wt tot }} \quad \mathrm{Wt} \% \text { NO3 }=6.5 \cdot \%
\end{aligned}
$$


Calcine weight using method in the report ENICO-1100 (Ninth Campaign, WCF):

Additional concentrations needed are negligible for the Newby test case:

$$
\begin{aligned}
& \mathrm{Ni} \equiv 0.0 \cdot \mathrm{mole} \quad \mathrm{Mo} \equiv 0.0 \cdot \mathrm{mole} \quad \mathrm{Mn} \equiv 0.0 \cdot \text { mole Gd } \equiv 0.0 \cdot \mathrm{mole} \quad \mathrm{Sn} \equiv 0.0 \cdot \mathrm{mole} \quad \mathrm{Nb} \equiv 0.0 \cdot \mathrm{mole} \\
& \text { Calcine_Wt } \text { old }:=\left[\begin{array}{l}
85.00 \cdot \mathrm{Na}+101.11 \cdot \mathrm{K}+50.98 \cdot \mathrm{Al}+123.22 \cdot \mathrm{Zr}+34.81 \cdot \mathrm{B}+79.85 \cdot \mathrm{Fe} \ldots \\
+76.00 \cdot \mathrm{Cr}+74.71 \cdot \mathrm{Ni}+143.94 \cdot \mathrm{Mo}+86.94 \cdot \mathrm{Mn}+181.25 \cdot \mathrm{Gd} \ldots \\
+150.69 \cdot \mathrm{Sn}-16.01 \cdot \mathrm{F}-5.02 \cdot \mathrm{PO} 4+110.09 \cdot \mathrm{Ca}+132.91 \cdot \mathrm{Nb} \ldots \\
+\mathrm{if}(\mathrm{Cd}>\mathrm{SO} 4,(80.06 \cdot \mathrm{SO}+128.40 \cdot \mathrm{Cd}),(182.41 \cdot \mathrm{Cd}+26.05 \cdot \mathrm{SO} 4))
\end{array}\right] \cdot \frac{\mathrm{gm}}{\mathrm{mole}}+\mathrm{UDS} \\
& \text { Calcine_Wt }{ }_{\text {old }}=216.7 \cdot \mathrm{gm} \quad \text { (of calcine formed per liter of solution) }
\end{aligned}
$$

Determine weight percent of $\mathrm{NO} 3$ in calcine for old method to see how it compares:

In the old method, $10 \%$ of the sodium plus potassium forms nitrates and $50 \%$ of the excess calcium forms $\mathrm{Ca}\left(\mathrm{NO}_{3}\right)_{2}$. For the test case, there is enough cadmium that all cadmium forms cadmium sulfate and no calcium sulfate forms. Fluoride left after formation of $\mathrm{Na}_{3} \mathrm{AlF}_{6}$ and $\mathrm{K}_{3} \mathrm{AlF}_{6}$. Excess calcium is that left after formation of $\mathrm{Ca}_{3}\left(\mathrm{PO}_{4}\right)_{2}, \mathrm{CaF}_{2}$, and $\mathrm{CaCl}_{2}$ :

$$
\begin{aligned}
& \mathrm{Ca}_{\mathrm{xs} \_ \text {old }}:=\mathrm{Ca}-\frac{3}{2} \cdot 0.5 \cdot \mathrm{PO} 4-\frac{1}{2} \cdot\left[\mathrm{F}-\frac{6}{3} \cdot(1-0.1) \cdot(\mathrm{Na}+\mathrm{K})\right]-\frac{1}{2} \cdot \mathrm{Cl} \quad \mathrm{Ca}_{\mathrm{xs} \_ \text {old }}=0.65 \cdot \mathrm{mole} \\
& \mathrm{NO}{ }_{\text {old }}:=0.1 \cdot(\mathrm{Na}+\mathrm{K})+0.5 \cdot(2) \cdot \mathrm{Ca} \mathrm{xs}_{-} \text {old } \quad \mathrm{NO}{ }_{\text {old }}=0.681 \cdot \mathrm{mole} \\
& \mathrm{Wt}_{\mathrm{NO}} \text { _old }:=62.005 \cdot \frac{\mathrm{gm}}{\mathrm{mole}} \cdot \mathrm{NO3} \text { old } \quad \mathrm{Wt}_{\mathrm{NO} 3 \text { old }}=\begin{array}{c}
42.208 \cdot \mathrm{gm} \text { (of NO3 formed per liter } \\
\text { of solution) }
\end{array} \\
& \text { Wt } \% \text { NO3_old }:=\frac{\mathrm{Wt} \text { NO3_old }}{\text { Calcine_Wt old }} \quad \text { Wt } \% \text { NO3_old }=19.5 \cdot \%
\end{aligned}
$$

For the test case, the old method overestimates the amount of nitrates by about:

$$
\mathrm{Wt}_{\text {NO3_old }}{ }^{-\mathrm{Wt}} \mathrm{NO}=29.8 \cdot \mathrm{gm}
$$

And the total weight of calcined solids differs from the updated method by:

Calcine_Wt old $^{- \text {Calcine_Wt }}$ tot $=25.9 \cdot \mathrm{gm}$ (of calcine formed per liter of solution)

$$
\text { or: } \frac{\text { Calcine_Wt } \text { old }- \text { Calcine_Wt tot }}{\text { Calcine_Wt tot }} \cdot 100 \%=13.6 \%
$$


Appendix E

Calculation of Liquid Waste Decay Heat

E-1 


\section{APPENDIX E \\ Calculation of Liquid and Calcine Waste Decay Heat}

The heat generation rates for calcine are calculated using the same method prepared by $R$. E. Schindler for Appendix $\mathrm{F}$ of Reference 1 except that decay energy values have been updated, and more radionuclides are included.

The decay heat generation of the liquid waste can be calculated by summing the decay heat contributions of each of the radionuclides whose concentrations are determined by sample analysis. The calculation procedure is outlined below and an example shown in Table E-1. The energy values in the table were obtained from D. R. Wenzel (Reference 15) who extracted them from ORIGIN2 version 2.1. Only prominent radionuclides in current tank farm waste are included in the table, and other radionuclides which contribute less than about $0.2 \%$ to the total decay energy were not included. Halflives are given in addition to decay energy so that measured concentrations of the individual nuclides can be adjusted for decay from the time of analysis to the proposed time of calcination. The products of the concentrations of the concentrations and decay heats are summed to get the total decay heat of the liquid, which is converted to watts per cubic meter. Multiplication by the volume reduction factor gives the decay heat gereration rate for the calcined solids.

Some of the less prominent nuclides will sometimes be omitted from an analytical report because their concentrations are below detection limits. The error introduced by these omissions is slight because the major radionuclides (Sr-90, Cs-134, Cs-137 and Ce-144) account for over $98 \%$ of the heat generation rate as shown at the bottom of the table.

The summing procedure is valid for current ICPP wastes which have aged several years (at least $2.5 \mathrm{yrs}$ ) out of reactor. With fresher waste, either additional nuclides should be included, or the ORIGIN2 code should be used for calculation of heat generation. Also, only short-lived daughters are included in the decay energy values, so if analytical data is more than a couple of years old ( $>2$ to 3 ), rather than using decayed values, $I$ would recommend either 1 ) using the undecayed concentrations to give a conservatively high heat generation rate, or 2) using the ORIGIN2 code to determine heat generation from decay. 


\begin{tabular}{|c|c|c|c|c|c|c|c|}
\hline & & Recoverable & $\begin{array}{l}\text { Table } \\
\text { cay Energy }\end{array}$ & $\begin{array}{l}-1 \\
\text { r Calcined }\end{array}$ & quid Waste & & \\
\hline Radio- & Half-Life & Q V & & $\begin{array}{r}\text { Waste } A \\
\text { WI }\end{array}$ & $\begin{array}{l}\text { lyses for } \\
180^{*}\end{array}$ & $\begin{array}{l}\text { WM-188/18 } \\
\text { High Sodi }\end{array}$ & $\begin{array}{l}\text { 3/189:ANN } \\
\text { m Blend** }\end{array}$ \\
\hline & (s) & (Mev/dis) & (Watts/Ci) & $(\mathrm{mCi} / \mathrm{L})$ & $\left(\mathrm{W} / \mathrm{m}^{3}\right)$ & $(\mathrm{mCi} / \mathrm{L})$ & $\left(\mathrm{W} / \mathrm{m}^{3}\right)$ \\
\hline Co-60 & $1.663 e+08$ & 2.601 & $1.542 \mathrm{e}-02$ & 0 & 0 & 0.5559 & 0.008571 \\
\hline Sr-Y-90 & $9.190 \mathrm{e}+08$ & 1.1308 & $6.704 \mathrm{e}-03$ & 25 & 0.167588 & 184.6615 & 1.237879 \\
\hline Ru-Rh-106 & $3.181 \mathrm{e}+07$ & 1.6280 & $9.651 \mathrm{e}-03$ & 0 & 0 & 0 & 0 \\
\hline Sb-125 & $8.741 \mathrm{e}+07$ & 0.5274 & $3.126 \mathrm{e}-03$ & 0 & 0 & 0 & 0 \\
\hline Cs-134 & $6.508 \mathrm{e}+07$ & 1.717 & $1.018 \mathrm{e}-02$ & 0.12 & 0.001221 & 3.4905 & 0.035528 \\
\hline $\mathrm{Cs}-\mathrm{Ba}-137$ & $9.467 e+08$ & 0.849 & $5.033 \mathrm{e}-03$ & 30 & 0.150989 & 200.6717 & 1.009974 \\
\hline Ce-Pr-144 & $2.456 e+07$ & 1.3519 & $8.014 e-03$ & 0 & 0 & 4.1703 & 0.033422 \\
\hline Eu-154 & $2.714 \mathrm{e}+08$ & 1.509 & $8.946 \mathrm{e}-03$ & 0.00078 & 0.000007 & 1.706 & 0.015261 \\
\hline Eu-155 & $1.565 e+08$ & 0.1227 & $7.274 \mathrm{e}-04$ & 0 & 0 & 1.2965 & 0.000943 \\
\hline $\mathrm{Pu}-238$ & $2.769 \mathrm{e}+09$ & 5.591 & $3.314 \mathrm{e}-02$ & 0 & 0 & 0.2205 & 0.007308 \\
\hline Pu-239 & $7.594 e+11$ & 5.199 & $3.082 \mathrm{e}-02$ & 0 & 0 & 0 & 0 \\
\hline $\mathrm{Am}-241$ & $1.364 e+10$ & 5.604 & $3.322 \mathrm{e}-02$ & 0.063 & 0.002093 & 0.0379 & 0.001259 \\
\hline & & & Sum $=$ & & 0.3219 & & 2.350 \\
\hline & & Sum & $\mathrm{Cr}+\mathrm{Cs}+\mathrm{Ce}=$ & & 0.3198 & & 2.317 \\
\hline & & $\overline{\mathrm{Sr}+\mathrm{Cs}+\mathrm{C}}$ & $\%$ of Total= & & $99.35 \%$ & & $98.58 \%$ \\
\hline
\end{tabular}

'Reference 21

"*References 19 and 20 


\section{Activity decay equation:}

$A=A_{0} e^{\left(-\frac{\ln 2}{t_{1 / 2}} t\right)}$

where: $A=$ Activity at time $t$, disintegrations $/ \mathrm{s}-\mathrm{mL}$ or $\mathrm{mCi} / \mathrm{L}$

$\mathrm{A}_{0}=$ Activity at analysis, disintegrations $/ \mathrm{s}-\mathrm{mL}$ or $\mathrm{mCi} / \mathrm{L}$

$\mathbf{t}=$ Time elapsed since analysis, seconds

$t_{1 / 2}=$ half life of radionuclide, seconds (or of parent radionuclide where daughter energies are included)

This is also sometimes represented as:

$$
A=A_{0} e^{(-\lambda t)}
$$

where: $\lambda=$ decay constant $\left(\sec ^{-1}\right)$

\section{Decay heat of liquid:}

$$
\mathrm{Q}_{\mathrm{liq}}\left(\mathrm{W} / \mathrm{m}^{3}\right)=\sum\left(\left(\mathrm{E}_{\text {decay }} \mathrm{W} / \mathrm{Ci}\right)\left(10^{-3} \mathrm{Ci} / \mathrm{mCi}\right)(\mathrm{A} \mathrm{mCi} / \mathrm{L})\left(10^{3} \mathrm{~L} / \mathrm{m}^{3}\right)\right)
$$

or

$$
\mathrm{Q}_{\text {liq }}\left(\mathrm{W} / \mathrm{m}^{3}\right)=\sum\left(\left(\mathrm{E}_{\text {decay }} \mathrm{Mev} / \mathrm{dis}\right)(\mathrm{A} \text { dis } / \mathrm{s}-\mathrm{mL})\right)\left(10^{6} \mathrm{~mL} / \mathrm{m}^{3}\right)\left(1.602 \cdot 10^{-13} \mathrm{~W}-\mathrm{s} / \mathrm{Mev}\right)
$$

\section{Decay heat of calcined solids:}

$$
\mathrm{Q}_{\text {cakine }}=\mathrm{Q}_{\mathrm{iq}} \mathrm{VRF}_{\mathrm{liq}}
$$

where: $V F_{\text {liq }}=$ Volume Reduction Factor, ratio of volume of feed blend or tank farm waste to volume of calcine produced 